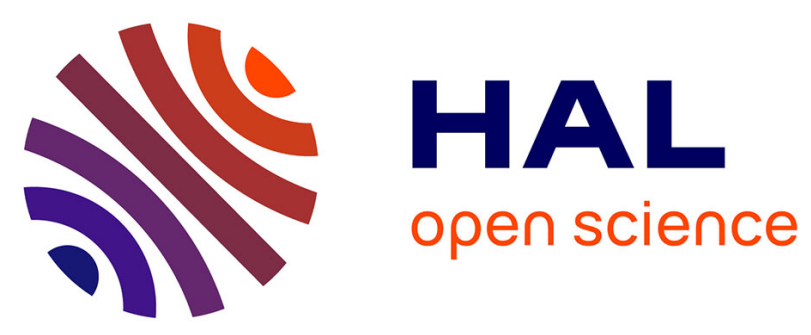

\title{
Census and classification of low-surface-brightness structures in nearby early-type galaxies from the MATLAS survey
}

Michal Bílek, Pierre-Alain Duc, Jean-Charles Cuillandre, Stephen Gwyn, Michele Cappellari, David V Bekaert, Paolo Bonfini, Theodoros Bitsakis, Sanjaya Paudel, Davor Krajnović, et al.

\section{To cite this version:}

Michal Bílek, Pierre-Alain Duc, Jean-Charles Cuillandre, Stephen Gwyn, Michele Cappellari, et al.. Census and classification of low-surface-brightness structures in nearby early-type galaxies from the MATLAS survey. MNRAS, 2020, 000 (2), pp.1 - 35. 10.1093/mnras/staa2248 . hal-02993421

\section{HAL Id: hal-02993421 \\ https://hal.science/hal-02993421}

Submitted on 6 Nov 2020

HAL is a multi-disciplinary open access archive for the deposit and dissemination of scientific research documents, whether they are published or not. The documents may come from teaching and research institutions in France or abroad, or from public or private research centers.
L'archive ouverte pluridisciplinaire HAL, est destinée au dépôt et à la diffusion de documents scientifiques de niveau recherche, publiés ou non, émanant des établissements d'enseignement et de recherche français ou étrangers, des laboratoires publics ou privés. 


\title{
Census and classification of low-surface-brightness structures in nearby early-type galaxies from the MATLAS survey
}

\author{
Michal Bílek ${ }^{1 \star}$, Pierre-Alain Duc ${ }^{1}$, Jean-Charles Cuillandre ${ }^{2}$, Stephen Gwyn ${ }^{3}$, \\ Michele Cappellari ${ }^{4}$, David V. Bekaert ${ }^{5}$, Paolo Bonfini ${ }^{6}$, Theodoros Bitsakis ${ }^{7}$, \\ Sanjaya Paudel ${ }^{8}$, Davor Krajnović ${ }^{9}$, Patrick R. Durrell ${ }^{10}$, Francine Marleau ${ }^{11}$ \\ 1 Université de Strasbourg, CNRS, Observatoire astronomique de Strasbourg (ObAS), UMR 7550, 67000 Strasbourg, France \\ 2 IRFU, CEA, Université Paris-Saclay, Université Paris Diderot, AIM, Sorbonne Paris Cité, CEA, CNRS, Observatoire de Paris, \\ PSL Research University, F-91191 Gif-sur-Yvette Cedex, France \\ ${ }^{3}$ NRC Herzberg Astronomy and Astrophysics, 5071 West Saanich Road, Victoria, BC, V9E 2E7, Canada \\ 4 Astrophysics, University of Oxford, Denys Wilkinson Building, Keble Road, Oxford, OX1 3RH, England \\ 5 MCG Department, Woods Hole Oceanographic Institution, Woods Hole, MA, USA \\ 6 University of Crete - Department of Physics, Voutes University Campus, GR-71003 Heraklion, Greece \\ 7 Institute of Astrophysics, FORTH, GR-71110 Heraklion, Greece \\ 8 Department of Astronomy and Center for Galaxy Evolution Research, Yonsei University, Seoul 03722, Korea \\ 9 Leibniz-Institut für Astrophysik Potsdam (AIP), An der Sternwarte 16, D-14482 Potsdam, Germany \\ 10 Department of Physics 8 Astronomy, Youngstown State University, Youngstown, OH 44555 USA \\ 11 Institute of Astro and Particle Physics, Technikerstrasse 25/8, University of Innsbruck, A-6020 Innsbruck, Austria
}

Accepted XXX. Received YYY; in original form ZZZ

\begin{abstract}
The morphology of galaxies gives essential constraints on the models of galaxy evolution. The morphology of the features in the low-surface-brightness regions of galaxies has not been fully explored yet because of observational difficulties. Here we present the results of our visual inspections of very deep images of a large volume-limited sample of 177 nearby massive early-type galaxies (ETGs) from the MATLAS survey. The images reach a surface-brightness limit of $28.5-29$ mag $\operatorname{arcsec}^{-2}$ in the $g^{\prime}$ band. Using a dedicated navigation tool and questionnaire, we looked for structures at the outskirts of the galaxies such as tidal shells, streams, tails, disturbed outer isophotes or peripheral star-forming disks, and simultaneously noted the presence of contaminating sources, such as Galactic cirrus. We also inspected internal sub-structures such as bars and dust lanes. We discuss the reliability of this visual classification investigating the variety of answers made by the participants. We present the incidence of these structures and the trends of the incidence with the mass of the host galaxy and the density of its environment. We find an incidence of shells, stream and tails of approximately $15 \%$, about the same for each category. For galaxies with masses over $10^{11} \mathrm{M}_{\odot}$, the incidence of shells and streams increases about 1.7 times. We also note a strong unexpected anticorrelation of the incidence of Galactic cirrus with the environment density of the target galaxy. Correlations with other properties of the galaxies, and comparisons to model predictions, will be presented in future papers.
\end{abstract}

Key words: galaxies: elliptical and lenticular, $\mathrm{cD}$ - galaxies: interactions - galaxies: peculiar - galaxies: haloes - galaxies:photometry - galaxies: structure

\section{INTRODUCTION}

Deep galaxy imaging of nearby extended systems is a dynamically growing part of present-day observational astron- omy. With current large-field-of-view cameras and dedicated observing techniques, we can get close to a surfacebrightness limit of $30 \mathrm{mag} \operatorname{arcsec}^{-2}$ in a few hours of observations or even less. Deep imaging is being used for multiple applications.

^ E-mail: bilek@astro.unistra.fr (MB) 
For instance, it can be employed to detect tidal features, the remnants of galaxy interactions. They can be either disturbances made by tidal forces exerted by one or more closeby companions in an on-going interaction, or be made of material that originally belonged to another galaxy. Tidal features are useful in many regards; for instance their incidence and morphology can then be compared to simulations to test galaxy formation theories (e.g., Bullock \& Johnston 2005; Johnston et al. 2008; Cooper et al. 2010; Hendel \& Johnston 2015; Pop et al. 2018; Mancillas et al. 2019), tidal features can help us clarify how unusual galaxies were formed (e.g., Sanders \& Mirabel 1996; Draper \& Ballantyne 2012; George 2017; Oh et al. 2019; Müller et al. 2019a; Ebrová et al. 2020) and they moreover are useful probes of gravitational fields (e.g., Ebrová et al. 2012; Sanderson \& Helmi 2013; Bílek et al. 2013, 2015a,b; Sanderson et al. 2017; Thomas et al. 2017, 2018; Malhan \& Ibata 2019), among others.

Deep imaging also discloses the extended stellar halos (e.g., Trujillo \& Bakos 2013; Trujillo \& Fliri 2016; Merritt et al. 2016; Mihos et al. 2017; Iodice et al. 2017b; Rich et al. 2019; Iodice et al. 2020) made of accreted material, i.e. dissolved tidal features, or that formed during the dissipationalcollapse stage of galaxy assembly. In specific cases, faint extended star-forming disks may be revealed, even around passive early type galaxies (Duc et al. 2015).

Detecting low-surface brightness galaxies on deep images became popular recently (Javanmardi et al. 2016; Greco et al. 2018; Mihos et al. 2018; Habas et al. 2019; Müller et al. $2019 b)$. The nature and formation of these galaxies is an interesting question by itself (e.g., Dabringhausen \& Kroupa 2013; Amorisco \& Loeb 2016; Di Cintio et al. 2017; Chan et al. 2018; Carleton et al. 2019; Liao et al. 2019); the dwarf galaxies also serve as test-beds for cosmological models (e.g., Kroupa et al. 2005; Müller et al. 2016; Libeskind et al. 2016; Banik \& Zhao 2018; Pawlowski et al. 2019; Javanmardi \& Kroupa 2020). Even though the first ultra-diffuse galaxies were discovered a long time ago (Sandage \& Binggeli 1984; Impey et al. 1988; Dalcanton et al. 1997), the new observing techniques helped to raise a strong interest of scientists in this type of galaxies (e.g., van Dokkum et al. 2015; Koda et al. 2015; Mihos et al. 2015; Román \& Trujillo 2017a,b; Papastergis et al. 2017; van Dokkum et al. 2018a,b; Bennet et al. 2018; González et al. 2018; Alabi et al. 2018; Torrealba et al. 2019; Janssens et al. 2019).

Finally, a fraction of the deep images capture galactic cirri, the diffuse part of the interstellar medium of our own galaxy. Although more prominent towards lower Galactic latitude, they are present in the whole sky and hinder the detection of low surface brightness objects behind them. One may take advantage of them studying the processes in the ISM at spatial scales inaccessible before (Miville-Deschênes et al. 2016; Román et al. 2019).

Deep images raise challenges at the observational and data-reduction stages, in particular related to the instrumental large-scale sky background variations, ghost halos and other PSF effects. These effects produce artifacts that can either be confused with the real astronomical objects, or make the detection of the objects of interest uncertain. The pitfalls of deep imaging are illustrated by the recent controversy about the tidal streams of the Splinter Galaxy, NGC 5907. Several amateur observers equipped by small telescopes have observed the streams warping the galaxy multiple times - the refereed example is Martínez-Delgado et al. (2008) - while the professional astronomers with bigger telescopes were able to detect only a part of this structure (Laine et al. 2016; van Dokkum et al. 2019; Müller et al. 2019c; Alabi et al. 2020). The case remains unresolved.

Once the images are reduced, we have to identify the structures of interest. Let us present the methods on the example of tidal features, the structures that have probably been investigated most in the past. The vast majority of existing works rely, at least partly, on the visual inspection of images whose brightness scale has been adjusted suitably (e.g., Bridge et al. 2010; Kaviraj 2010; Nair \& Abraham 2010; Sheen et al. 2012; Atkinson et al. 2013). Others employ more elaborate methods that may include removing point-like sources and smoothing the image by a Gaussian or a median kernel filter (e.g., Tal et al. 2009; Miskolczi et al. 2011; Adams et al. 2012; Morales et al. 2018; Hood et al. 2018), unsharp masking technique (see the pioneering work by Malin \& Carter 1983) and subtraction of galaxy models (McIntosh et al. 2008) that enhance the visibility of faint streams and shells. For detecting tidal features in large galaxy samples, automated methods have to be employed. Kado-Fong et al. (2018) preselected galaxies for visual inspection using an algorithm based on detecting high spatial frequencies in the image. Another method relies on the tidal parameter, a function of the average ratio of the original image and a smooth model of the galaxy (van Dokkum 2005; Adams et al. 2012). Pawlik et al. (2016) developed a method based on the asymmetry of isophotes. The coefficients such as concentration, asymmetry, Gini or $M_{20}$ (Abraham et al. 1996; Conselice 2003; Lotz et al. 2004) work well for detecting perturbed galaxies at high redshift, nevertheless they are not suitable for detecting tidal features whose luminosity comprises just a small fraction of the total luminosity of the galaxy. We do not just wish to know if tidal features are present - their morphological type and number provide further precious constraints. The automatic algorithms listed so far cannot do that in contrast with the visual inspection. Promising methods for both detecting and classifying galaxy substructures are convolutional neural networks (Walmsley et al. 2019; Pearson et al. 2019), that reach an $\sim 80 \%$ completeness with a $\sim 20 \%$ contamination or the algorithm called "Subspace-Constrained Unsupervised Detection of Structure" (Hendel et al. 2019). The importance of the automated methods will grow with the advent of the unprecedented number of new images produced by the large future surveys, see Sect. 6 .

The price to pay for deep imaging is the requirement of relatively long observing time coupled with the need to observe large number of systems. A number of observing efforts have been performed to achieve this. We compiled the characteristics of some of the ongoing, recent or notable surveys looking for tidal features or exploring low-surface brightness structures in Table 1. Note that we have not included in this list the limiting surface brightness. Indeed, different authors use different methods to estimate it making comparisons difficult. Differences may be as large as $2-3$ mag from one method to the other, depending on whether integrated profiles or local measurements are made. Instead, we state whether the survey was optimized for detecting extended low-surface-brightness structures, i.e. that the largedithering strategy was employed (see, e.g., Duc et al. 2015). 
In our work we exploit deep optical images of a complete volume-limited sample of 177 nearby ETGs taken in the MATLAS survey (Duc et al. 2015; Habas et al. 2019). All galaxies studied here are a part of the ATLAS ${ }^{3 \mathrm{D}}$ reference sample (Cappellari et al. 2011a) meaning that a lot of additional information is available about them and hence it will be possible to study the relations of the faint structures with other properties of the galaxies. In addition, the originality of the MATLAS survey, compared to similar studies, lies in the combination of depth, LSB optimization, the number of target galaxies, detailed classification methods and image quality. The excellent seeing at CFHT and the large diameter of the telescope provide images having a much better angular resolution than $<1 \mathrm{~m}$ telescopes.

In this paper we present a catalogue of the following types of structures/features/objects in or around the MATLAS ETGs: 1) tidal features (streams, shells, tails), 2) disturbed outer isophotes, 3) stellar bars and other features formed by secular evolution of rotating galaxies, 4) dust lanes, 5) peripheral star-forming disks, and finally 6) galactic cirri. We investigate their incidence, and for the tidal features also their number in the galaxy. Finally, we explore how the incidence correlates with the mass and environmental density of the galaxies, which are important properties influencing the evolution of galaxies. Since our work will be a basis for subsequent works within the ATLAS ${ }^{3 \mathrm{D}}$ project, we provide a detailed discussion of the possible biases of the method we used for our structure detection and classification.

The paper is organized as follows. In Sect. 2 we give details about our sample and the MATLAS survey. Our methods are explained in Sect. 3 and the results are presented in Sect. 4. Section 5 is the discussion where we consider the advantages and shortcomings of the method (Sect. 5.1), the comparison of our results with literature (Sect. 5.2) and provide a qualitative interpretation of the results (Sect. 5.3). Section 6 deals with our future plans. We summarize in Sect. 7. Supplementary information is given in the appendices. Our methods to classify the galaxies are described more in detail in Appendix A. In Appendix B, we explored how experience of the participants affects their classifications of the galaxies. Finally, in Appendix $\mathrm{C}$ we provide example images of the various types of features of interest for this paper.

\section{THE MATLAS SURVEY}

The images used in this paper were obtained as part of the MATLAS deep imaging project carried out at the $3.6 \mathrm{~m}$ Canada-France-Hawaii Telescope (CFHT) using the MegaCam imager. The instrument combines the light gathering power of a relatively big telescope with a wide fieldof-view. The images reach local surface brightness limit of 28.5-29 $\mathrm{mag} \mathrm{arcsec}^{-2}$ in the $g^{\prime}$ band (Duc et al. 2015). The large field-of-view of $1 \times 1^{\circ}$ allows detecting extended tidal structures around nearby galaxies as well as inspecting their environment, including the presence of contaminating cirrus emission at large scales. The latter usually appears as parallel stripes, which might be confused with tidal features if the field-of-view is not large enough.

The MATLAS project developed as a CFHT Large Pro- gram made in the framework of the ATLAS ${ }^{3 \mathrm{D}}$ collaboration (Cappellari et al. 2011a). As such, it benefited from the availability of the multi-wavelength spectroscopic and imaging observations with various instruments and telescopes (Sauron/WHT, Westerbork radio telescope, IRAM, etc.). The acquisition of the deep images was completed by short-exposure observations of the galaxies whose inner regions were saturated. The details are described in Duc et al. (2015), together with preliminary results based on the observations of a fraction of the sample presented here. An exhaustive description of the full MATLAS survey, including the field positions and observing conditions, is presented in Habas et al. (2019). Table 2 summarizes the main characteristics of the survey.

The reference sample for this study, the ATLAS ${ }^{3 \mathrm{D}}$ sample with 260 members, is characterized in Cappellari et al. (2011a). Briefly, it is a complete volume-limited sample of nearby galaxies (distance below $42 \mathrm{Mpc}$ ), that are massive (absolute $K$-band magnitude below -21.5), easily observable from the northern hemisphere $\left(\left|\delta-29^{\circ}\right|<35^{\circ}\right)$, that avoid the obscuration by the Milky Way $\left(|b|>15^{\circ}\right)$ and were visually classified as early-type, based on shallow optical images from the Sloan Digital Sky Survey (Abazajian et al. 2009) or the Isaac Newton Telescope. The MATLAS sub-sample was obtained from the ATLAS ${ }^{3 \mathrm{D}}$ sample by excluding the 58 galaxies belonging to the Virgo cluster, since they have already had deep images from the "Next Generation Virgo Cluster Survey" (Ferrarese et al. 2012) when the project was conceived. Therefore MATLAS does not probe the densest environments since all ATLAS ${ }^{3 \mathrm{D}}$ galaxies in clusters reside in Virgo. An analysis similar as the one presented here for the ATLAS ${ }^{3 \mathrm{D}}$ galaxies in Virgo is planned. Furthermore, the MATLAS sample does not include galaxies that are located close to bright stars as they are unsuitable for deep imaging (about 20 of them). The final MATLAS sample analyzed in the present paper includes 177 ETGs for which at least two bands $\left(g^{\prime}\right.$ and $\left.r^{\prime}\right)$ are available. Additional $i^{\prime}$ band and $u^{*}$ band images were obtained for resp. $60 \%$ and $7 \%$ of this sample.

The primary objective of MATLAS is the systematic census of the relics of past collisions, i.e. tidal features and extended stellar halos. While the first type of structures is discussed in the current paper, exploiting the images of stellar halos requires corrections for the light scattered by the optical elements of the camera, and the wide wings of the PSF. A deconvolution technique is used for this (Karabal et al. 2017). Results will presented in Yıldız et al. in prep.. The study of diffuse extended star-forming disks traced by blue and UV emission or dust lanes has been detailed in Yildız et al. (2017) and Yildız et al. (2020). The census of such relatively rare features around ETG is also presented in the present paper.

Because of the large field-of-view of the MegaCam camera, the MATLAS survey allows studying the large-scale environment of the target ETGs: massive spiral companions (about 100 of them), but also dwarf galaxy satellites, including ultra-diffuse galaxies, and associated globular clusters (GCs), which are also good tracers of the past assembly of galaxies. Habas et al. (2019) described a sample of about 2200 dwarf candidates from the MATLAS images. A catalog of GC candidates in MATLAS images was compiled by Lim et al. in prep. The association between GCs and 
Table 1. Comparison of MATLAS to other surveys targeting tidal features or low-surface-brightness objects.

\begin{tabular}{|c|c|c|c|c|c|c|c|c|}
\hline Paper (survey name) & $\begin{array}{l}\text { LSB opti- } \\
\text { mized? }\end{array}$ & $\begin{array}{l}\text { Coverage } \\
{\left[\mathrm{deg}^{2}\right]}\end{array}$ & Bands & Telescope & $\begin{array}{l}\mathrm{FOV}^{\mathrm{g}} \\
{\left[\mathrm{deg}^{2}\right]}\end{array}$ & Targets & $\begin{array}{l}\text { Number } \\
\text { of objects }\end{array}$ & $\begin{array}{l}\text { Distance } \\
{[\mathrm{Mpc}]}\end{array}$ \\
\hline $\begin{array}{l}\text { This work } \\
\text { (MATLAS) }\end{array}$ & $\mathrm{Y}$ & 144 & $\left(u^{*}\right) g^{\prime} r^{\prime}\left(i^{\prime}\right)^{\mathrm{f}}$ & $3.6 \mathrm{~m} \mathrm{CFHT}$ & 1 & ETGs & 177 & $<40$ \\
\hline $\begin{array}{l}\text { Annibali et al. (2020) } \\
(\mathrm{SSH})\end{array}$ & $\mathrm{Y}$ & 6.6 & $g r$ & $11.9 \mathrm{~m} \mathrm{LBT}$ & 0.15 & LTG dwarfs & 45 & $\lesssim 10$ \\
\hline $\begin{array}{l}\text { Danieli et al. (2019) } \\
\left(\text { DWFS }^{\text {a })}\right.\end{array}$ & $\mathrm{Y}$ & $330^{\mathrm{d}}$ & $g r$ & 1m Dragonfly & blind & - & - & - \\
\hline $\begin{array}{l}\text { Gilhuly et al. (2019) } \\
\left(\text { DEGS }^{\mathrm{a}}\right)\end{array}$ & $\mathrm{Y}$ & - & - & 1m Dragonfly & 4.9 & Edge-on LTGs & - & - \\
\hline $\begin{array}{l}\text { Rich et al. (2019) } \\
\left(\text { HERON }^{\mathrm{a}}\right)\end{array}$ & $\mathrm{Y}$ & 68 & $L$ & $\begin{array}{l}0.7 \mathrm{~m} \text { Jeanne } \\
\text { Rich }\end{array}$ & 0.57 & Nearby gxs. & 119 & $<50$ \\
\hline $\begin{array}{l}\text { Hood et al. (2018) } \\
\text { (RESOLVE) }\end{array}$ & $\mathrm{N}+\mathrm{Y}$ & 710 & $r$ & $\begin{array}{l}4 \mathrm{~m} \mathrm{CTIO}+ \\
2.5 \mathrm{~m} \text { Sloan }\end{array}$ & blind & $\begin{array}{l}M_{*+\mathrm{HI}} \\
\gtrsim 10^{9.2} \mathrm{M}_{\odot}\end{array}$ & 1048 & $\sim 60-100^{\mathrm{b}}$ \\
\hline $\begin{array}{l}\text { Byun et al. (2018) } \\
\left.\text { (KMNet }^{\text {a }}\right)\end{array}$ & $\mathrm{Y}$ & - & - & $1.6 \mathrm{KMNet}$ & 4 & - & - & - \\
\hline $\begin{array}{l}\text { Kado-Fong et al. } \\
(2018)\left(\text { HSC-SSP}^{\mathrm{a}}\right)\end{array}$ & $\mathrm{N}$ & $200^{\mathrm{c}} / 1400^{\mathrm{d}}$ & $i^{\mathrm{c}}$ & $8.2 \mathrm{~m}$ Subaru & blind & all & 21208 & $200-2500^{\mathrm{b}}$ \\
\hline $\begin{array}{l}\text { Morales et al. (2018) } \\
\text { (SDSS) }\end{array}$ & $\mathrm{N}$ & 74.3 & $g+r+i$ & 2.5m Sloan & blind & $M_{*}=10^{10-11} \mathrm{M}_{\odot}$ & 297 & $\lesssim 30$ \\
\hline $\begin{array}{l}\text { Martínez-Delgado } \\
\text { (2019) }\left(\mathrm{STSS}^{\mathrm{a}, \mathrm{e}}\right)\end{array}$ & - & - & $L$ & $\begin{array}{l}0.1-0.5 \mathrm{~m} \\
\text { amateurs }\end{array}$ & $>0.25$ & $M_{K}<-19.6$ & - & $<40$ \\
\hline $\begin{array}{l}\text { Peters et al. }(2017) \\
\left(\text { SDSS Stripe } 82^{\mathrm{i}}\right)\end{array}$ & $\mathrm{Y}$ & 275 & ugriz & 2.5m Sloan & blind & Face-on LTGs & 22 & mostly $<100$ \\
\hline $\begin{array}{l}\text { Mihos et al. (2017) } \\
\text { (BSDVS) }\end{array}$ & $\mathrm{Y}$ & 16 & $B V_{M}$ & $\begin{array}{l}0.61 \mathrm{~m} \text { Burrell } \\
\text { Schmidt }\end{array}$ & blind & Virgo Cluster & - & - \\
\hline $\begin{array}{l}\text { Iodice et al. (2016, } \\
\text { 2017a) (FDS) }\end{array}$ & $\mathrm{Y}$ & 26 & ugri & $2.6 \mathrm{~m} \mathrm{VST}$ & blind & Fornax Cluster & - & 20 \\
\hline $\begin{array}{l}\text { Muñoz et al. (2015) } \\
\left(\text { NGFS }^{\mathrm{h}}\right)\end{array}$ & $\mathrm{Y}$ & 30 & $u^{\prime} g^{\prime} r^{\prime} i^{\prime} J K_{S}$ & $4 \mathrm{~m}$ CTIO & blind & Fornax Cluster & - & - \\
\hline $\begin{array}{l}\text { Capaccioli et al. } \\
(2015)\left(\text { VEGAS }^{\mathrm{a}}\right)\end{array}$ & Part & $\sim 100$ & gri & $2.6 \mathrm{~m} \mathrm{VST}$ & 0.9 & ETGs $B<-19.2$ & $\sim 100$ & $<60^{\mathrm{b}}$ \\
\hline $\begin{array}{l}\text { Atkinson et al. } \\
\text { (2013) (CFHTLS) }\end{array}$ & $\mathrm{N}$ & 170 & $g^{\prime}+r^{\prime}+i^{\prime}$ & $3.6 \mathrm{~m} \mathrm{CFHT}$ & 1 & $\begin{array}{l}r^{\prime}<15.5 \\
\left(M_{r^{\prime}}<19.3\right)\end{array}$ & 1781 & $180-690^{\mathrm{b}}$ \\
\hline $\begin{array}{l}\text { Ferrarese et al. } \\
(2012) \text { (NGVS) }\end{array}$ & $\mathrm{Y}$ & 104 & $u^{*} g(r) i z^{\mathrm{f}}$ & $3.6 \mathrm{~m} \mathrm{CFHT}$ & 1 & Virgo Cluster & - & - \\
\hline $\begin{array}{l}\text { Adams et al. (2012) } \\
\text { (MENeaCS) }\end{array}$ & $\mathrm{N}$ & 54 & $r$ & $3.6 \mathrm{~m} \mathrm{CFHT}$ & 1 & ETGs $M_{r}<-20$ & 3551 & $180-720^{\mathrm{b}}$ \\
\hline Sheen et al. (2012) & $\mathrm{N}$ & $\sim 1.5$ & $u g r$ & $4 \mathrm{~m}$ CTIO & blind & $\begin{array}{l}\text { red cluster-gxs. } \\
M_{r}<-20\end{array}$ & 273 & $200-520^{\mathrm{b}}$ \\
\hline $\begin{array}{l}\text { Miskolczi et al. } \\
\text { (2011) (SDSS DR7) }\end{array}$ & $\mathrm{N}$ & 8423 & $g+r+i$ & 2.5m Sloan & blind & edge-on LTGs & 474 & $\begin{array}{l}\text { mostly }< \\
100\end{array}$ \\
\hline $\begin{array}{l}\text { Kaviraj (2010)(SDSS } \\
\text { Stripe } 82)\end{array}$ & $\mathrm{N}$ & 270 & $r$ & $2.5 \mathrm{~m}$ Sloan & blind & $\begin{array}{l}\text { ETGs } \\
M_{r}<-20.5\end{array}$ & $\sim 300$ & $\lesssim 220^{\mathrm{b}}$ \\
\hline $\begin{array}{l}\text { Nair \& Abraham } \\
(2010)(\text { SDSS DR4) }\end{array}$ & $\mathrm{N}$ & 6670 & $g^{\prime} r^{\prime} i^{\prime}$ & 2.5m Sloan & blind & $g<16$ & 14034 & $40-460^{b}$ \\
\hline $\begin{array}{l}\text { Bridge et al. (2010) } \\
\text { (CFHTLS-Deep) }\end{array}$ & $\mathrm{N}$ & 2 & $u^{*} g^{\prime} r^{\prime} i^{\prime} z^{\prime}$ & $3.6 \mathrm{~m} \mathrm{CFHT}$ & blind & $i_{\text {Vega }} \leq 22.2$ & 27000 & $\begin{array}{l}990- \\
84000^{\mathrm{b}}\end{array}$ \\
\hline $\begin{array}{l}\text { Tal et al. (2009) } \\
\text { (OBEY) }\end{array}$ & $\mathrm{Y}$ & $\sim 6$ & $V$ & $1 \mathrm{~m}$ CTIO & 0.11 & Es, $M_{B}<-20.15$ & 55 & $15-50$ \\
\hline $\begin{array}{l}\text { van Dokkum (2005) } \\
\text { (MUSYC, NDWF) }\end{array}$ & $\mathrm{N}$ & 10.5 & $\begin{array}{l}U B V R I z, \\
B_{w} R I\end{array}$ & $\begin{array}{l}\text { 4m Mayall, } \\
4 \mathrm{~m} \text { CTIO }\end{array}$ & blind & ETGs & 126 & mean $7500^{\mathrm{b}}$ \\
\hline $\begin{array}{l}\text { Malin \& Carter } \\
(1983)\end{array}$ & $\mathrm{N}$ & - & $\begin{array}{l}\text { IIIa-J } \\
\text { emulsion }\end{array}$ & $\begin{array}{l}1.24 \mathrm{~m} \mathrm{UK} \\
\text { Schmidt }\end{array}$ & 44 & ETGs & 137 & $\begin{array}{l}\text { majority }< \\
300\end{array}$ \\
\hline
\end{tabular}

Notes: ${ }^{a}$ Ongoing surveys. ${ }^{\mathrm{b}}$ Estimated from redshift assuming $H_{0}=69.6 \mathrm{~km} \mathrm{~s}^{-1}, \Omega_{\mathrm{m}}=0.286$ and flat cosmology as the luminosity distance using the calculator of Wright (2006). ${ }^{\mathrm{c}}$ Used in the paper. ${ }^{\mathrm{d}}$ Intended final coverage. ${ }^{\mathrm{e}}$ See also Martínez-Delgado et al. (2009). ${ }^{\mathrm{f}}$ Images in the bands listed in parenthesis were available only for a part of the investigated galaxies. " The "blind" fields mean that the target is

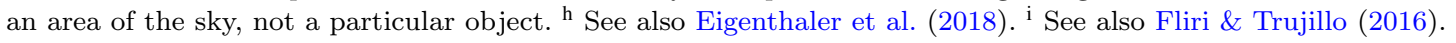



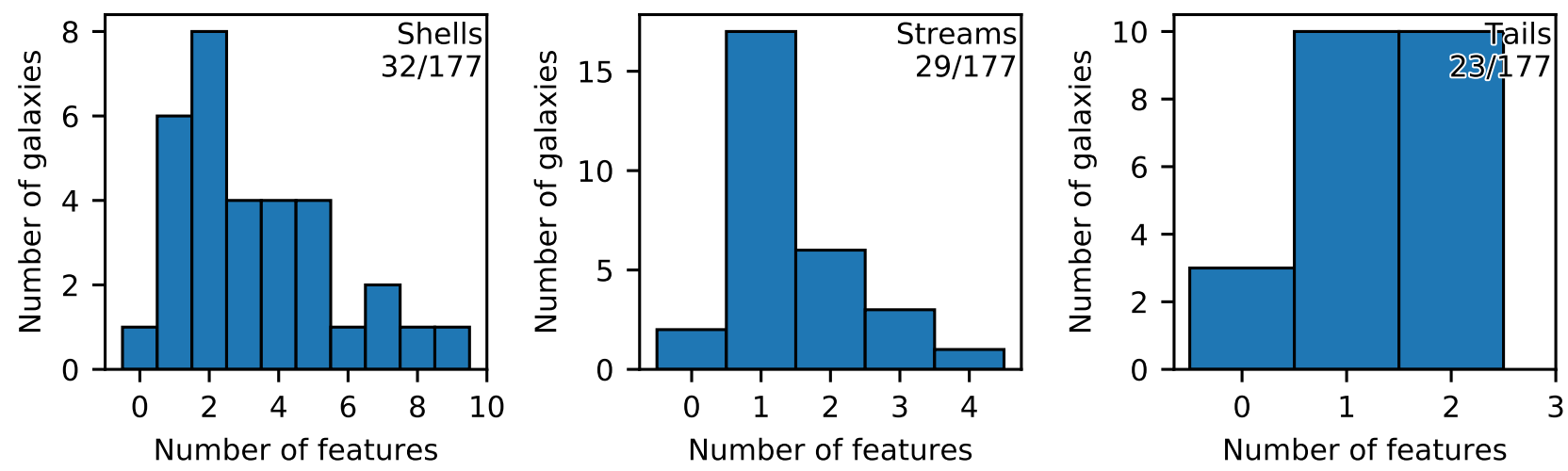

Figure 1. Histograms of the number of shells (left), streams (middle) and tails (right) per galaxy in our sample. Here are included only the galaxies where the number of detected features was greater than zero (the galaxies in the zero bin have, e.g., 0.5 streams, i.e. one likely stream, see Appendix A4). The number of galaxies that were counted in in these histograms and the total number of galaxies in the sample are noted in the corners.

Table 2. Survey characteristics of MATLAS.

\begin{tabular}{ccccc}
\hline \hline & $u^{*}$ & $g^{\prime}$ & $r^{\prime}$ & $i^{\prime}$ \\
\hline Number of observed galaxies & 12 & 178 & 179 & 104 \\
Average seeing [arcsec] & 1.03 & 0.96 & 0.84 & 0.65 \\
\hline
\end{tabular}

some collisional features for one system is presented in Lim et al. (2017) and Fensch et al. 2020 (submitted). In principle, it will be possible to estimate the dynamical mass of the galaxies in our sample from the number of their GCs (e.g., Harris et al. 2015; Forbes et al. 2018) or kinematics (e.g., Samurović 2014; Bílek et al. 2019b) after measuring the radial velocities of the GCs. In this paper however, our mass dependence analysis makes use of a proxy of the stellar mass.

A fraction of the MATLAS images capture Galactic cirri, dust clouds in our own Milky Way, some located close to the Sun. Miville-Deschênes et al. (2016) illustrated the great scientific potential of such images for studying the turbulence cascade in the interstellar medium at high spatial resolution. Fields affected by cirrus are systematically annotated in this paper.

\section{METHODS}

We give here a brief description of our fine structure identification and classification methods; they are detailed in Appendix A. The results of this paper are based on visual inspection of the MATLAS images. The images were inspected by six scientists, all specialists in observing or simulating nearby galaxies. Each of them classified at least twothirds of the galaxies, and the majority all of them. Clearly, the number is low compared to citizen-science projects such as Galaxy Zoo (Lintott et al. 2008), but this is compensated by a much stronger expertise of our classifiers. In fact, the initial group of participants included more people (15). An analysis of the votes had shown a decrease of the degree of consensus when the votes of the less experienced classifiers were included.

The participants were provided an online navigation tool for displaying the images that allowed them to navigate through the images, zoom in and out, change the lu- minosity scaling, bands, etc. This tool and all images used for the classification is made available publicly at http: //obas-matlas.u-strasbg.fr/. The participants were invited to fill a questionnaire about the presence, number and prominence of several types of features in or around the galaxies. Namely, we were interested in the following features (example images provided in Appendix $\mathrm{C}$ and on the MATLAS public web site):

- The tidal features, i.e. tails, streams and shells. These structures inform us about the gravitational interactions that the target galaxy had, or is having, with other galaxies. Tails refer to structures whose material seems to come from the galaxy being classified. This means that it is either an ongoing interaction or remnant of a major merger and then the mass of the accreted galaxy constitutes a substantial fraction of the mass of target galaxy. Streams refer to structures apparently formed by a tidal disruption of a much smaller companion galaxy. Shells are azimuthal arcs centered on the core of the host galaxy; they are characterized by sharp outer edges. Such features are known to form in minor to intermediate nearly radial mergers (Quinn 1983; Bílek et al. 2015a; Pop et al. 2018). The measure of the frequency of each type of tidal features, combined with knowledge of their specific life times and detectability as a function of time and viewing angles, estimated from simulations (Mancillas et al. 2019), can give us constrains on the past merging history of galaxies, and help to quantify the fraction of stellar mass gathered by mergers of various types. We should point out that this approach is valid for a given cosmological model. For instance, assuming modified gravity, many tidal features are expected to have arisen from non-merging galaxy flybys (Bílek et al. 2018, 2019a).

- The shape of the outermost isophote of the target galaxy. Strongly disturbed isophotes trace recent interactions and are usually associated with tidal features. Mildly irregular isophotes can be the last witnesses of old galaxy interactions when tidal features have already faded out. Alternatively, mild isophotal irregularities can indicate weak or just starting interactions.

- Peripheral disks. A characteristic of an ETG is an old red stellar population. Deep images however reveal that 
some ETGs are surrounded by faint disks of young blue stars, providing clues on the rejuvenation of ETGs.

- The features induced by secular evolution (bars, rings, spiral arms). Such features are usually located in the bright parts of the galaxy and thus shallow images are usually good enough for their detection. However they had not been previously identified in a systematic way in MATLAS. Our deep data can reveal the exceptional cases of the secular features in the low-surface brightness regions.

- Dust lanes and patches. Such features can form spontaneously in the interstellar medium of ETGs but also trace past gas-rich merging events. The MATLAS deep images can reveal dust regions in the outer stellar halo that are not detectable in shallower images.

- Galactic cirrus. Scattering the optical light of nearby stars, these dust clouds trace the most diffuse interstellar medium of our own galaxy. They can complicate the detection and identification of the extragalactic structures under study, but are also interesting targets for detailed ISM studies at high angular resolution.

In addition, two contributors logged the presence of close-by polluting halos that could have plausibly affected the detection or classification of the structures of interest. We considered two types of such halos: the instrumental ghosts surrounding bright stars and caused by internal reflections in the camera (see Figures C7-C9 for examples), and the stellar halos of neighboring galaxies, whose isophotes overlap with those of the target.

The answers of individual participants regarding a particular feature had to be converted to a single real number, the so-called rating of the feature. Initially, we converted the answers about the presence of the features in numbers, e.g. "no", "likely" and "yes" to 0, 1 and 2, respectively. The final rating was chosen as the option that got most votes by the participants. If several answers got the same number of votes, the rating is the average of the most frequent answers. The rating of presence of a feature can range from 0 , corresponding to absence of the feature, to 2 , signifying the presence, with the exception of peripheral disks, whose ratings range between 0 and 1 . We assigned the galaxies a numerical rating of halos of 1 if the galaxies were affected by halos, or 0 otherwise. For some purposes, e.g., when we wanted to count how many galaxies have a certain type of feature, we had to round the rating to discrete categories. Then we speak about the "rounded rating".

\section{RESULTS}

\subsection{Final classification}

The ratings of the individual galaxies are presented in Table D1. Each galaxy was assigned a morphological code consistently with Duc et al. (2015) based on the rounded rating of the presence of the classified features in the galaxy. The meaning of the code is explained in Table 3. For example, the code " $+\mathrm{s}+\mathrm{d}+\mathrm{ph}+\mathrm{pl}$ " for IC 1024 means: the galaxy contains streams (the " $+\mathrm{s}$ " symbol) but no shells, tails or bar (there are no symbols signifying these features); the outer isophote has a perturbed shape (the " + ph" symbol); the main body shows prominent dust lanes (the "+pl" symbol), and there is no cirrus in the field, or nearby halos polluting the image
Table 3. Explanation of the classification codes.

\begin{tabular}{|c|c|}
\hline Code & Meaning \\
\hline \multicolumn{2}{|r|}{ Features } \\
\hline symbol alone & the feature is present \\
\hline symbol with "?" & the feature is likely / unsure \\
\hline no symbol & the feature is missing \\
\hline$+\mathrm{s}$ & Streams \\
\hline$+\mathrm{r}$ & shells / Ripples \\
\hline$+\mathrm{t}$ & Tails \\
\hline$+\mathrm{d}$ & peripheral star-forming Disk \\
\hline$+a h$ & Asymmetric outer isophotes / stellar Halo \\
\hline$+\mathrm{ph}$ & $\begin{array}{l}\text { disturbed / Perturbed outer isophotes } \\
\text { / stellar Halo }\end{array}$ \\
\hline$+\mathrm{wl}$ & Weak dust Lanes \\
\hline$+\mathrm{pl}$ & strong / Prominent dust Lanes \\
\hline$+w b$ & Weak Bars \\
\hline$+\mathrm{pb}$ & strong / Prominent Bars \\
\hline \multicolumn{2}{|r|}{ Contaminants } \\
\hline$-\mathrm{h}$ & polluting Halos \\
\hline$-\mathrm{wc}$ & Weak Cirrus \\
\hline$-\mathrm{pc}$ & strong / Prominent Cirrus \\
\hline
\end{tabular}

(the corresponding symbols are missing). In Appendix $\mathrm{C}$ we present images of the galaxies that exhibit the most prominent examples of the classified structure types.

\subsection{Statistics of the structures}

Here we highlight some of the main results obtained from the statistical analysis of the visual classification. A detailed analysis, including the correlations between the frequency of the classified structures with the internal properties of the galaxies, the comparisons with predictions from numerical simulations, and the conclusions about the past mass assembly of the target galaxies, will be presented in future papers of this series.

The results of our census of the investigated features for our sample according to the rounded rating are presented in Table 5. It shows the incidence the classified feature types in our sample along with the Poisson errors.

One can see that shells, streams and tails have about the same incidence appearing in about $10-15 \%$ of galaxies. The more general indicator of tidal interactions, the irregularity of the outer isophotes, turns out to be more frequent. Isophotes are disturbed or asymmetric in about $30 \%$ of the galaxies. Tidal structures however often appear together. Any of tails, streams and shells are at least likely in $30 \%$ of galaxies. When we include galaxies with disturbed or asymmetric isophotes we detected signs of tidal interactions in about $40 \%$ of galaxies. The distinction between steams and tails can be ambiguous. When these two categories are merged, we obtain that at least likely signs of them were detected in 20-25\% of galaxies. The bars were rated at least as weak in about $35 \%$ of the sample. Dust lanes were detected in about $15 \%$ of galaxies. Peripheral disks are the least frequent type of structures among those we investigated, appearing just in a few percent of all galaxies. Our census indicates that $40 \%$ of the galaxies in our sample are not affected by the presence of polluting halos (from companions or bright stars). Galactic cirri, might have directly affected the classification of $10-20 \%$ of galaxies.

We made also statistics of the number of tidal features 
Table 4. Ratings and classification codes of the individual galaxies. The complete table is available in Appendix D.

\begin{tabular}{lllllllllll}
\hline \hline Name & Classification code & Streams & Shells & Tails & Ext. SF & Out. isoph. & Dust & Bars & Halos & Cirrus \\
\hline IC 0560 & + wb & 0.0 & 0.0 & 0.0 & 0.0 & 0.0 & 0.0 & 1.0 & 0.0 & 0.0 \\
IC 0598 & & 0.0 & 0.0 & 0.0 & 0.0 & 0.0 & 0.0 & 0.0 & 0.0 & 0.0 \\
IC 0676 & $+\mathrm{pb}$ & 0.0 & 0.0 & 0.0 & 0.0 & 0.0 & 0.0 & 2.0 & 0.0 & 0.0 \\
IC 1024 & $+\mathrm{s}+\mathrm{ph}+\mathrm{pl}$ & 2.0 & 0.0 & 0.0 & 0.0 & 2.0 & 2.0 & 0.0 & 0.0 & 0.0 \\
NGC 0448 & & 0.0 & 0.0 & 0.0 & 0.0 & 0.0 & 0.0 & 0.0 & 0.0 & 0.0 \\
NGC 0474 & $+\mathrm{s}+\mathrm{r}+\mathrm{t}+\mathrm{ph}-\mathrm{h}$ & 2.0 & 2.0 & 2.0 & 0.0 & 2.0 & 0.0 & 0.0 & 1.0 & 0.0 \\
NGC 0502 & $+\mathrm{r}+\mathrm{ah}$ & 0.0 & 2.0 & 0.0 & 0.0 & 1.0 & 0.0 & 0.0 & 0.0 & 0.0 \\
NGC 0509 & $+\mathrm{pb}$ & 0.0 & 0.0 & 0.0 & 0.0 & 0.0 & 0.0 & 2.0 & 0.0 & 0.0 \\
NGC 0516 & $+\mathrm{wb}$ & 0.0 & 0.0 & 0.0 & 0.0 & 0.0 & 0.0 & 1.0 & 0.0 & 0.0 \\
NGC 0524 & $+\mathrm{h}$ ?+wl-pc & 0.0 & 0.5 & 0.0 & 0.0 & -1 & 1.0 & 0.0 & 0.0 & 2.0 \\
\hline
\end{tabular}

Table 5. Census of the classified structures in the whole sample according to the rounded rating (in percent).

\begin{tabular}{lllll}
\hline \hline Shells & no: $84 \pm 7$ & likely: $5 \pm 2$ & yes: $12 \pm 3$ & unknown: $0 \pm 0$ \\
Streams & no: $84 \pm 7$ & likely: $5 \pm 2$ & yes: $11 \pm 2$ & unknown: $0 \pm 0$ \\
Tails & no: $87 \pm 7$ & likely: $3 \pm 1$ & yes: $10 \pm 2$ & unknown: $0 \pm 0$ \\
Outer isophotes & regul.: $67 \pm 6$ & asym.: $12 \pm 3$ & disturb.: $20 \pm 3$ & unsure: $0.6 \pm 0.6$ \\
Tails or streams & no: $76 \pm 7$ & likely: $5 \pm 2$ & yes: $19 \pm 3$ & unknown: $0 \pm 0$ \\
Shells or streams or tails & no: $70 \pm 6$ & likely: $7 \pm 2$ & yes: $23 \pm 4$ & unknown: $0 \pm 0$ \\
Any tidal disturbance & no: $59 \pm 6$ & likely: $13 \pm 3$ & yes: $28 \pm 4$ & unknown: $0 \pm 0$ \\
Bars & no: $64 \pm 6$ & weak: $20 \pm 3$ & strong: $16 \pm 3$ & unsure: $0 \pm 0$ \\
Dust lanes & no: $85 \pm 7$ & weak: $7 \pm 2$ & strong: $8 \pm 2$ & unsure: $0 \pm 0$ \\
Peripheral disks & no: $96 \pm 7$ & & yes: $4 \pm 1$ & unsure: $0 \pm 0$ \\
Halos & no: $71 \pm 6$ & & yes: $29 \pm 4$ & unsure: $0 \pm 0$ \\
Cirrus & no: $83 \pm 7$ & weak: $7 \pm 2$ & strong: $10 \pm 2$ & unsure: $0 \pm 0$ \\
\hline
\end{tabular}

per galaxy in Fig. 1. The median number of the individual tidal-feature types is 2.71 for shells, 1.0 for streams, and 1.25 for tails where we have constrained ourselves just to the galaxies having the rating of the presence of the given feature greater than zero (galaxies in the zeroth bin contain, for example, one likely stream, i.e. the rating was 0.5 ).

Theoretical arguments lead us to expect (see Sect. 5.3) that the frequency of the investigated morphological structures should depend on the mass of the galaxy and the density of its environment. We therefore evaluated how the frequency of fine structure depends on the quantities $M_{\mathrm{JAM}}$ and $\rho_{10}$. The first of them, $M_{\mathrm{JAM}}$, is the dynamical mass obtained by Jeans Anisotropic Modeling (Cappellari 2008) within the sphere of a radius of one projected half-light radius as derived by Cappellari et al. (2013) from the observed kinematic maps of the galaxies. Cappellari et al. (2013) and Poci et al. (2017) calculated a median dark matter fraction of $13 \%$ for the ATLAS ${ }^{3 \mathrm{D}}$ sample. Given the small fraction of dark matter needed, the mass $M_{\mathrm{JAM}}$ is then a better estimator for the stellar mass than the estimates based on stellar population synthesis because of the uncertainties of stellar evolution, initial mass function and dust obscuration. The environment density $\rho_{10}$ is defined as the mean density of galaxies inside a sphere centred on the galaxy and containing 10 nearest neighbours (Cappellari et al. 2011b). One might argue that mass and environment are closely related as more massive galaxies usually reside in denser environments but Fig. 2 demonstrates that $M_{\mathrm{JAM}}$ and $\rho_{10}$ are little correlated for our sample and that the incidence of the classified features depends on both quantities. After presenting the detected correlations in the rest of this section, we interpret them in Sect. 5.3.

Figures 3 and 4 show how the frequency of the individual types of the classified structures depend on $M_{\mathrm{JAM}}$ and $\rho_{10}$ of the galaxy, respectively. The widths of the bins were set to contain equal number of galaxies. The two galaxies for which $M_{\mathrm{JAM}}$ is not available, PGC 058114 and PGC 071531, were excluded.

In addition, we made a statistical test of the significance of the correlations based on Spearman's rank coefficient, $r$, that is designed to be $1(-1)$ for a strictly increasing (decreasing) sequence of data. The use of this coefficient is motivated by the visual impression of monotonic trends in Figs. 3 and 4, e.g. the correlation between the occurrence of shells and the mass of the galaxy. The results are shown in Table 6. Here we also give the corresponding $p$-value, i.e. the probability that the absolute value of $r$ is greater than the observed $r$ if there is actually no correlation between the occurrence of the feature with the property of the host galaxy. The values $r_{\text {all }}$ and $p_{\text {all }}$ were calculated for the whole sample. There are also trends in Figs. 3 and 4 that suggest a more complicated relationship, e.g. the peak in the occurrence of shells in the medium density bin or the vanishing of the correlation between the occurrence of disturbed outer isophotes in the medium and low mass bins. We therefore divided the galaxy sample into two halves depending on whether the mass (environment density) of the galaxy was greater or smaller than the median mass (environment density) and applied the statistical test to each half; the median values are 10.60 for $\log M_{\mathrm{JAM}} / M_{\odot}$ and -1.64 for $\log \rho_{10} /\left(\mathrm{Mpc}^{-3}\right)$. We list the results for the lower (higher) half of the sample in Table 6 as $r_{1 \mathrm{~h}}$ and $p_{1 \mathrm{~h}}\left(r_{2 \mathrm{~h}}\right.$ and $\left.p_{2 \mathrm{~h}}\right)$. The $p$-values that signify an inconsistency with no correlation at the $5 \%$ confidence level are highlighted by boldface. One should look for a theoretical explanation of such correlations.

In Fig. 3 we can see that tidal features are more frequent in more massive galaxies. The correlations for streams, shells and perturbed isophotes are statistically significant 

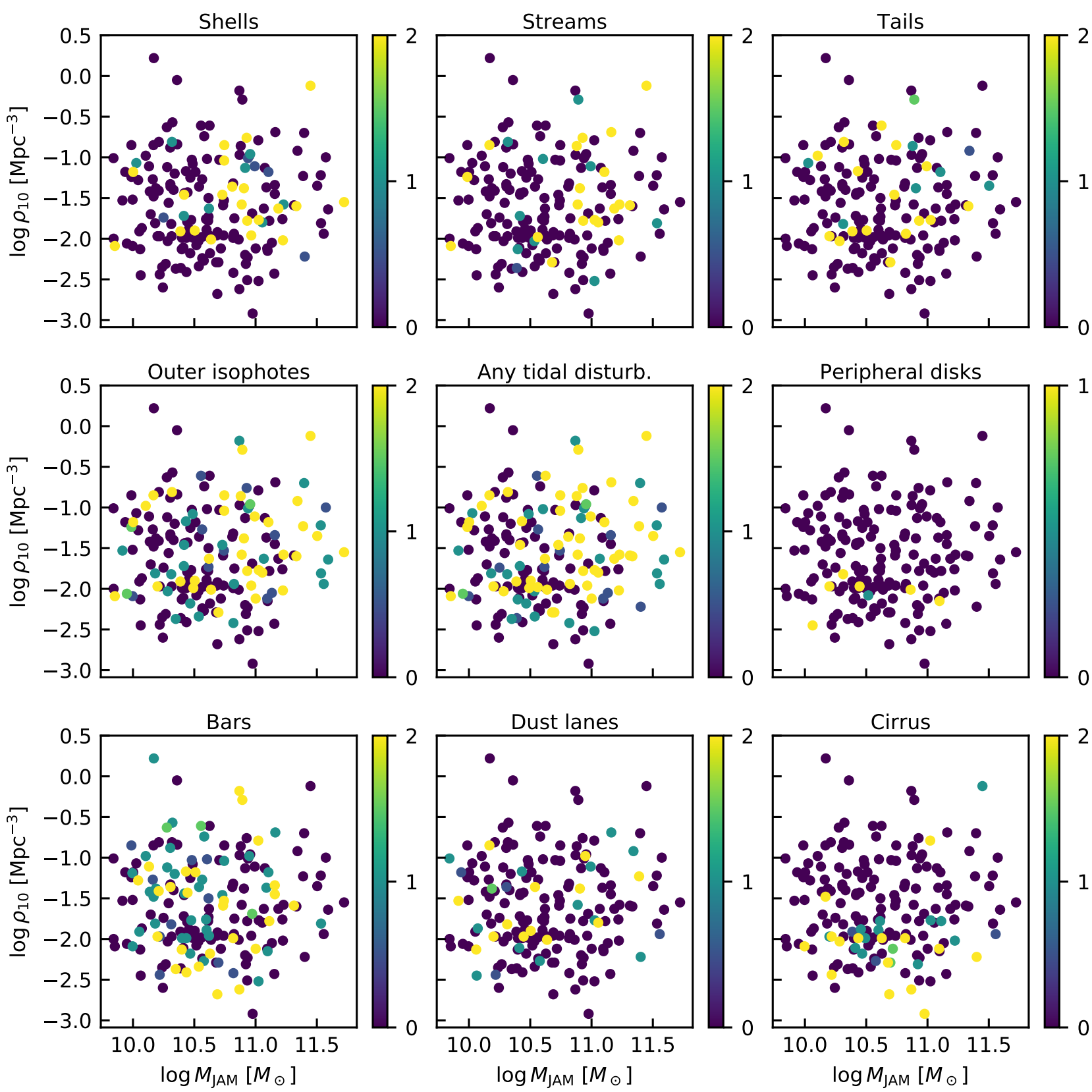

Figure 2. Occurrence of the investigated features depending on the $M_{\mathrm{JAM}}$ mass of the galaxy and the density of its environment $\rho_{10}$. The color scale indicates the rating of the presence or prominence of the given feature in the particular galaxy.

while that for the tidal tails is not as we can learn from Table 6 . The field "Any tidal disturbance" indicates whether the galaxy has either shells, streams, tails or irregular isophotes; more precisely this quantity is defined as the maximum of the ratings of these morphological features. There is a visual appearance of an abrupt boost of the incidence of streams, disturbed isophotes or tidal disturbances in general at high masses. The confidence of this feature misses our adopted confidence threshold of $5 \%$. There are also no significant correlations of the incidence of bars, dust lanes, and, as expected, foreground cirrus with the mass of the galaxy.

Table 7 provides the census of the fine structures for galaxies over the mass of $10^{11} \mathrm{M}_{\odot}$. There are 35 such galax- ies. Comparison to the census in the complete sample in Table 5 yields that the incidence of shells, streams and disturbed isophotes increases, for these massive galaxies, by factors of $1.4 \pm 0.5,2.0 \pm 0.6$ and $1.6 \pm 0.4$, respectively.

In Fig. 4 we present the correlations of the incidence of our structures of interest with the environment density of the target galaxy, probed by the $\rho_{10}$ parameter. The feature that correlates the strongest with the galaxy environment is surprisingly the presence of cirrus in our own Galaxy. The lower galaxy density, the more probable the cirrus occurrence is. We discuss the possible explanations of this unexpected effect in Sect. 5. There is also a statistically significant correlation of the environment density with the inci- 

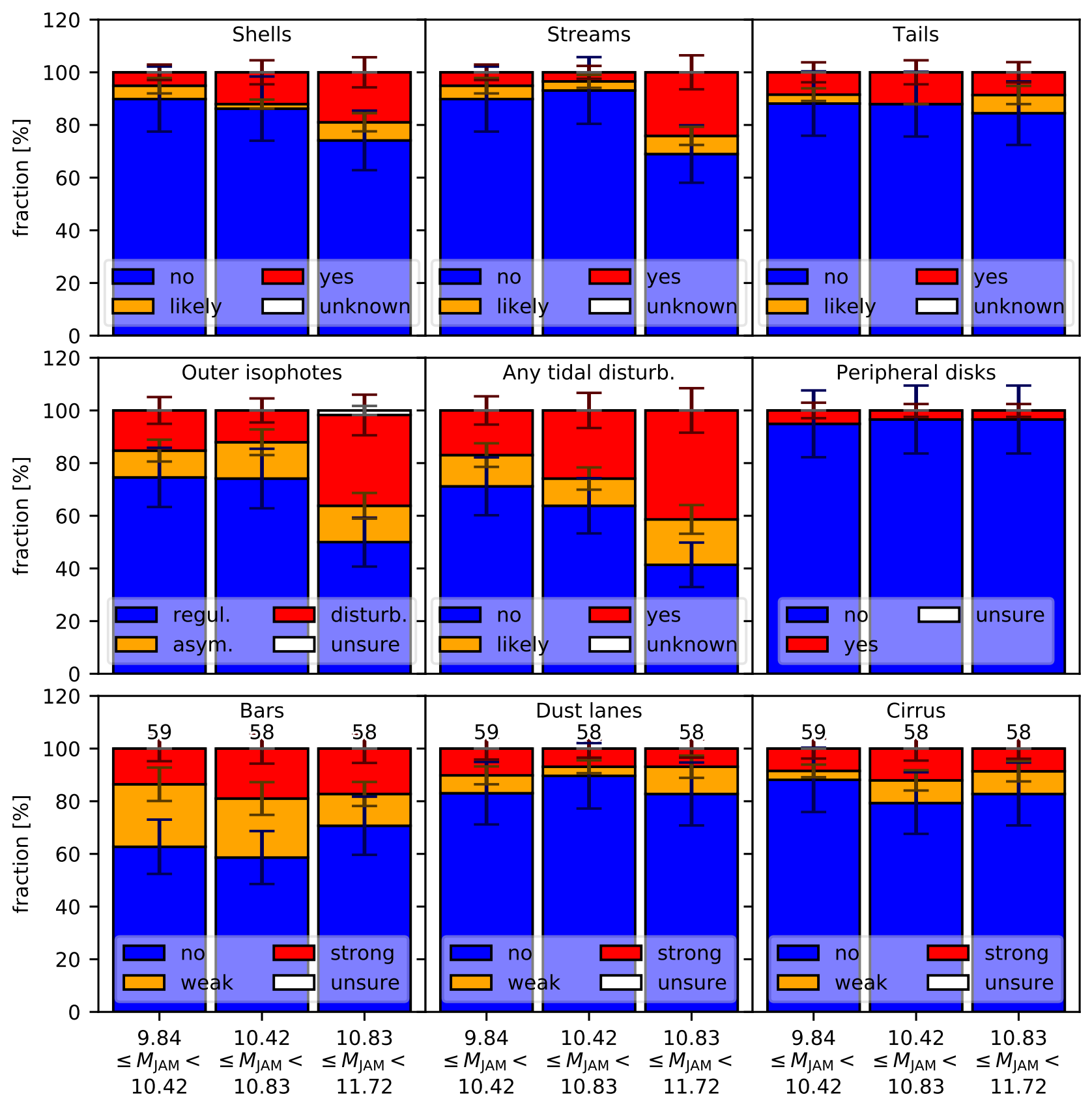

Figure 3. Fraction of the ratings of a given feature as a function of the $M_{\mathrm{JAM}}$ mass of the galaxy $\left(\right.$ units $\left.\log \left(M_{\mathrm{JAM}} / M_{\odot}\right)\right)$. The rounded rating was used. The numbers over the bins in the bottom row of the panels denote the numbers of galaxies in the bins. They were equalized on purpose.

dence of tails. On the contrary, the incidence of peripheral disks decreases significantly with increasing the density of the environment. Apart from this, the shell incidence increases significantly with $\rho_{10}$ in the low-density half of the sample. The decrease of the incidence of shells between the medium and high density bins is not confirmed by the test. Neither are the apparent correlations between the incidence of streams and disturbed isophotes with $\rho_{10}$. The correlation of $\rho_{10}$ with the union of all tidal disturbances closely misses our significance threshold.

Table 8 provides the census of the fine structures for the 45 galaxies residing in environments whose densities are below $10^{-2} \mathrm{Mpc}^{-3}$. By comparing to Table 5, one can find that the incidence of shells and tails is, compared to the whole sample, lower by a factor of $0.4 \pm 0.2$ and $0.3 \pm 0.2$, respectively.

Dust lanes and bars do not show any correlation with environment density of the host. We recall here that our sample does not include cluster galaxies, i.e. the galaxies in the densest environments.

Besides mass and environment, the incidence of the features studied here might correlate with other properties of the galaxy, such as the specific angular momentum, gas con- 

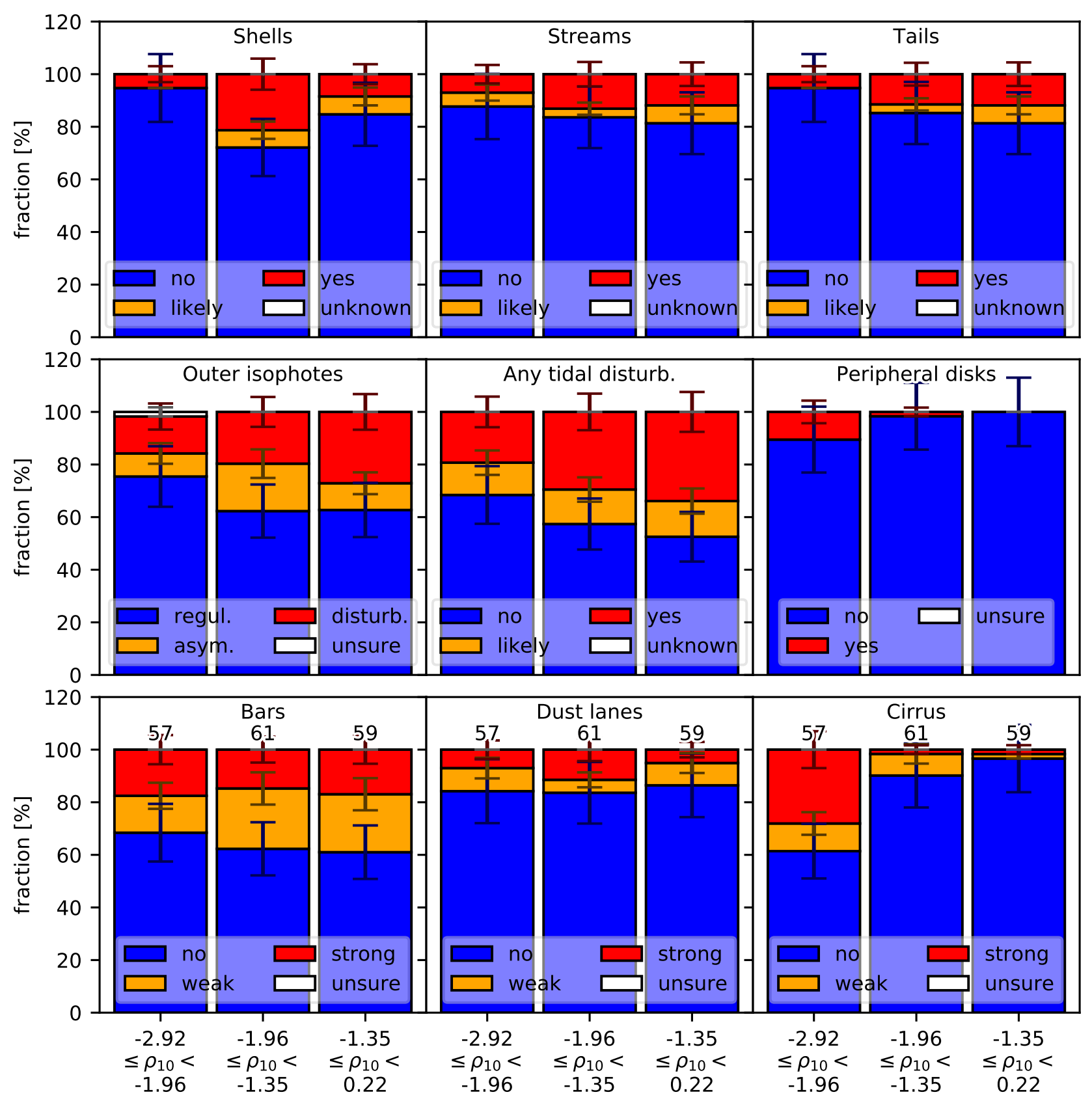

Figure 4. Fraction of the ratings of given feature as a function environment density of galaxies $\rho_{10}\left(u n i t s \log \left(\rho_{10} / \mathrm{Mpc}{ }^{-3}\right)\right.$ ). The rounded rating was used. The numbers over the bins in the bottom row of panels denote the numbers of galaxies in the bins. They were equalized on purpose.

tent, presence or absence of a core, etc. These will be studied in a future paper.

\section{DISCUSSION}

\subsection{Discussion of our method}

As already pointed out in the Introduction, the visual classification of the faint structure is necessarily subjective. Different people can classify a given feature as a tail or stream or these could be confused with Galactic cirrus. Nevertheless, before objective automatic algorithms for structure detec- tion and classification improve, visual identification remains the best option.

Our adopted rating procedure has several desirable properties. Since it includes voting, it eliminates the mistakes of individuals. We based our results on the votes of the participants who inspected at least two-thirds of the galaxies. Such classifiers had already a substantial experience with controlling the navigation tool, visualizing the faint structures, and distinguishing tails and streams, for example. We made a few tests to verify this expectation, see Appendix B. As the disadvantage, the voting method 
Table 6. Correlations of the occurrence the classified structures with the mass and environmental density of the host galaxy. The symbol $r$ stands for Spearman's rank coefficient and $p$ for the probability that $r$ is greater than the given value if there is actually no correlation. The subscript all stands for the complete sample while $1 h$ and $2 h$ stand, respectively, for the correlation between the first and second half of the sample when sorted with respect to the mass or environment density. The $p$-values that are inconsistent with no correlation at the $5 \%$ confidence level are highlighted by bold font.

\begin{tabular}{lllllll}
\hline \hline Quantities & $r_{\text {all }}$ & $p_{\text {all }}$ & $r_{\text {lh }}$ & $p_{\text {lh }}$ & $r_{2 \mathrm{~h}}$ & $p_{2 \mathrm{~h}}$ \\
\hline Shells $-\log M_{\text {JAM }}$ & 0.22 & $\mathbf{0 . 0 0 3 1}$ & -0.053 & 0.63 & 0.11 & 0.29 \\
Streams $-\log M_{\text {JAM }}$ & 0.21 & $\mathbf{0 . 0 0 4 9}$ & 0.017 & 0.87 & 0.20 & 0.064 \\
Tails $-\log M_{\text {JAM }}$ & 0.074 & 0.33 & -0.012 & 0.91 & -0.017 & 0.88 \\
Out. iso. $-\log M_{\text {JAM }}$ & 0.27 & $\mathbf{0 . 0 0 0 2 8}$ & -0.035 & 0.74 & 0.32 & $\mathbf{0 . 0 0 2 3}$ \\
Any tidal $\operatorname{disturb.~}-\log M_{\text {JAM }}$ & 0.30 & $\mathbf{0 . 0 0 0 0 4 1}$ & 0.026 & 0.81 & 0.26 & $\mathbf{0 . 0 1 6}$ \\
Peripheral $\operatorname{disks}-\log M_{\text {JAM }}$ & -0.064 & 0.40 & 0.028 & 0.80 & 0.018 & 0.87 \\
Secular $\mathrm{f} . \log M_{\mathrm{JAM}}$ & -0.092 & 0.23 & 0.12 & 0.26 & -0.14 & 0.21 \\
Dust $-\log M_{\mathrm{JAM}}$ & -0.063 & 0.40 & -0.10 & 0.35 & 0.20 & 0.067 \\
Cirrus $-\log M_{\mathrm{JAM}}$ & 0.073 & 0.34 & 0.070 & 0.52 & -0.073 & 0.50 \\
Shells $-\log \rho_{10}$ & 0.14 & 0.065 & 0.27 & $\mathbf{0 . 0 1 3}$ & -0.14 & 0.19 \\
Streams $-\log \rho_{10}$ & 0.080 & 0.29 & 0.073 & 0.50 & 0.066 & 0.54 \\
Tails $-\log \rho_{10}$ & 0.16 & $\mathbf{0 . 0 3 5}$ & 0.20 & 0.058 & 0.14 & 0.20 \\
Out. iso. $-\log \rho_{10}$ & 0.13 & 0.090 & 0.18 & 0.091 & 0.013 & 0.91 \\
Any tidal $\operatorname{disturb.}-\log \rho_{10}$ & 0.14 & 0.064 & 0.099 & 0.36 & -0.011 & 0.92 \\
Peripheral $\operatorname{disks}-\log \rho_{10}$ & -0.18 & $\mathbf{0 . 0 1 4}$ & -0.021 & 0.85 & nan & nan \\
Secular $\mathrm{f} .-\log \rho_{10}$ & 0.067 & 0.37 & -0.14 & 0.19 & 0.010 & 0.93 \\
Dust $-\log \rho_{10}$ & -0.015 & 0.85 & 0.027 & 0.81 & -0.024 & 0.82 \\
Cirrus $-\log \rho_{10}$ & -0.37 & $\mathbf{0 . 0 0 0 0 0 0 3 5}$ & -0.18 & 0.10 & 0.10 & 0.33 \\
\hline
\end{tabular}

Table 7. Census of the classified structures in the whole sample according to the rounded rating (in percent) but only for the galaxies whose $M_{\mathrm{JAM}}$ mass exceeds $10^{11} \mathrm{M}_{\odot}$.

\begin{tabular}{lllll}
\hline \hline Shells & no: $80 \pm 10$ & likely: $6 \pm 4$ & yes: $17 \pm 7$ & unknown: $0 \pm 0$ \\
Streams & no: $70 \pm 10$ & likely: $6 \pm 4$ & yes: $26 \pm 9$ & unknown: $0 \pm 0$ \\
Tails & no: $90 \pm 20$ & likely: $6 \pm 4$ & yes: $6 \pm 4$ & unknown: $0 \pm 0$ \\
Outer isophotes & regul.: $50 \pm 10$ & asym.: $17 \pm 7$ & disturb.: $30 \pm 10$ & unsure: $3 \pm 3$ \\
Tails or streams & no: $60 \pm 10$ & likely: $9 \pm 5$ & yes: $29 \pm 9$ & unknown: $0 \pm 0$ \\
Shells or streams or tails & no: $60 \pm 10$ & likely: $9 \pm 5$ & yes: $31 \pm 9$ & unknown: $0 \pm 0$ \\
Any tidal disturbance & no: $40 \pm 10$ & likely: $20 \pm 8$ & yes: $40 \pm 10$ & unknown: $0 \pm 0$ \\
Bars & no: $70 \pm 10$ & weak: $17 \pm 7$ & strong: $17 \pm 7$ & unsure: $0 \pm 0$ \\
Dust lanes & no: $80 \pm 20$ & weak: $11 \pm 6$ & strong: $6 \pm 4$ & unsure: $0 \pm 0$ \\
Peripheral disks & no: $100 \pm 20$ & & yes: $3 \pm 3$ & unsure: $0 \pm 0$ \\
Cirrus & no: $80 \pm 20$ & weak: $11 \pm 6$ & strong: $9 \pm 5$ & unsure: $0 \pm 0$ \\
\hline
\end{tabular}

Table 8. Census of the classified structures in the whole sample according to the rounded rating (in percent) but only for the galaxies whose environmental density is below $\log _{10} \rho_{10} /\left(1 \mathrm{Mpc}^{-3}\right)=-2$.

\begin{tabular}{lllll}
\hline \hline Shells & no: $90 \pm 10$ & likely: $0 \pm 0$ & yes: $7 \pm 4$ & unknown: $0 \pm 0$ \\
Streams & no: $90 \pm 10$ & likely: $7 \pm 4$ & yes: $7 \pm 4$ & unknown: $0 \pm 0$ \\
Tails & no: $100 \pm 10$ & likely: $0 \pm 0$ & yes: $4 \pm 3$ & unknown: $0 \pm 0$ \\
Outer isophotes & regul.: $70 \pm 10$ & asym.: $11 \pm 5$ & disturb.: $13 \pm 5$ & unsure: $2 \pm 2$ \\
Tails or streams & no: $80 \pm 10$ & likely: $7 \pm 4$ & yes: $11 \pm 5$ & unknown: $0 \pm 0$ \\
Shells or streams or tails & no: $80 \pm 10$ & likely: $7 \pm 4$ & yes: $13 \pm 5$ & unknown: $0 \pm 0$ \\
Any tidal disturbance & no: $70 \pm 10$ & likely: $16 \pm 6$ & yes: $18 \pm 6$ & unknown: $0 \pm 0$ \\
Bars & no: $70 \pm 10$ & weak: $13 \pm 5$ & strong: $18 \pm 6$ & unsure: $0 \pm 0$ \\
Dust lanes & no: $80 \pm 10$ & weak: $11 \pm 5$ & strong: $4 \pm 3$ & unsure: $0 \pm 0$ \\
Peripheral disks & no: $90 \pm 10$ & & yes: $9 \pm 4$ & unsure: $0 \pm 0$ \\
Cirrus & no: $60 \pm 10$ & weak: $9 \pm 4$ & strong: $27 \pm 8$ & unsure: $0 \pm 0$ \\
\hline
\end{tabular}

eliminates correct identifications of indistinct structures by sensitive individuals.

We explored the influence of the image pollutants, the polluting halos and cirri, in Table 9. It shows the census of our structures of interest but calculated only considering the galaxies whose images were not polluted much, namely the rating of the polluting halos was 0 and the rating of cirrus was $<1.5$. Comparing Table 9 to Table 5 one can see that there is no significant difference. This suggests that these pollutants do not affect our classification substantially.

At the time of the census, model subtracted images were not available for the majority of our galaxies. It is very likely that we missed some tidal features because of this. Tidal features can be hard to detect in the central parts where the luminosity of the underlying galaxy has a steep gradient. For this reason, the number of tidal features is very likely underestimated. On the other hand, our deep images enabled 
Table 9. Census of the classified structures according to the rounded rating (in percent). Only galaxies without strong pollutants were considered (rating of polluting halos $=1$, rating of cirrus $<1.5$ ).

\begin{tabular}{lllll}
\hline \hline Shells & no: $89 \pm 9$ & likely: $2 \pm 1$ & yes: $9 \pm 3$ & unknown: $0 \pm 0$ \\
Streams & no: $87 \pm 9$ & likely: $3 \pm 2$ & yes: $11 \pm 3$ & unknown: $0 \pm 0$ \\
Tails & no: $95 \pm 9$ & likely: $3 \pm 2$ & yes: $3 \pm 2$ & unknown: $0 \pm 0$ \\
Outer isophotes & regul.: $73 \pm 8$ & asym.: $12 \pm 3$ & disturb.: $15 \pm 4$ & unsure: $0 \pm 0$ \\
Tails or streams & no: $76 \pm 7$ & likely: $5 \pm 2$ & yes: $19 \pm 3$ & unknown: $0 \pm 0$ \\
Shells or streams or tails & no: $70 \pm 6$ & likely: $7 \pm 2$ & yes: $23 \pm 4$ & unknown: $0 \pm 0$ \\
Any tidal disturbance & no: $59 \pm 6$ & likely: $13 \pm 3$ & yes: $28 \pm 4$ & unknown: $0 \pm 0$ \\
Bars & no: $59 \pm 7$ & weak: $24 \pm 5$ & strong: $17 \pm 4$ & unsure: $0 \pm 0$ \\
Dust lanes & no: $82 \pm 9$ & weak: $7 \pm 3$ & strong: $11 \pm 3$ & unsure: $0 \pm 0$ \\
Peripheral disks & no: $96 \pm 9$ & & yes: $4 \pm 2$ & unsure: $0 \pm 0$ \\
\hline
\end{tabular}

us to detect well the structures in the outer parts of the galaxies that could be overlooked in the standard shallow images.

\subsection{Comparison to literature}

In this section we compare our results to other works.

\subsubsection{Tidal structures}

Table 1 of Atkinson et al. (2013) provides a useful compilation of tidal feature occurrence from 12 different works. The fraction of galaxies with tidal features varies a lot, from 3 to over $70 \%$. This is probably not only a result of different instruments, selection criteria, image depths and image processing techniques, but also of different criteria on the prominence of the feature. For example, Atkinson et al. (2013) give for their own results several degrees of confidence that a galaxy contains a tidal feature. Constraining themselves on the red galaxies in the highest confidence category, they found tidal features in $15 \%$ of galaxies, while counting in all red galaxies with any signs of tidal features, they arrived to $41 \%$. The work of Atkinson et al. (2013) is similar to ours since they used a visual identification on images obtained with the same instrument. They however processed and stacked differently, with a method not optimized for LSB studies. Their morphological categories differ from ours. One has to compare our category of shells with the union of their categories of shells, fans and diffuse structures; some of the features classified as the diffuse structures by Atkinson et al. (2013) would however be classified by us as galaxies with disturbed outer isophotes. Constraining ourselves just to their red galaxies and their two highest-confidence detection levels, their sample contains $12 \%$ of shell galaxies. This is consistent with the measured likely or secure shell occurrence of $17 \pm 5 \%$ in the MATLAS sample. This is more than Malin \& Carter (1983) who detected shells in around $10 \%$ of their galaxies, probably because their limiting surface brightness was shallower by about $2 \mathrm{mag} / \mathrm{arcsec}^{-2}$. In contradiction with us, Malin \& Carter (1983) found that the frequency of shells decreases with an increasing environment density. We detected a hint of peak of shell occurrence in the medium density bin but the decrease toward the high densities is not statistically significant. This might be explained by the fact that our sample does not include galaxies in clusters.

It seems the most relevant to compare the union of our categories of streams and tails with the union of the structures called streams, linear and arms in the red galaxies of Atkinson et al. (2013). The comparison yields $24 \pm 7 \%$ of these types of tidal features in our sample versus $14 \pm 1 \%$ in Atkinson et al. (2013). Besides the difference in the data reduction technique between the two surveys, one of the main reasons of this inconsistency is probably the larger average distance to their galaxies that made the detectability of these thin and faint structures harder. Indeed, the median redshift of their red galaxies is 1.4, i.e. around $500 \mathrm{Mpc}$, while our galaxies lie in the median distance of only $27.2 \mathrm{Mpc}$. Moreover, it seems from the example images provided by Atkinson et al. (2013) that some of their structures classified as "diffuse" would likely be classified as tails by us. Shell detectability is probably not affected that much by the distance perhaps because shells are more sharp-edged and are thus more easy to detect.

We detected a tidal disturbance of any type in $41 \pm$ $7 \%$ of galaxies, counting in also the likely detections. Red galaxies in the sample of Atkinson et al. (2013) have some form of a tidal disturbance in $22 \pm 2 \%$. We attribute this to the larger distances to the galaxies in Atkinson et al. (2013) since a lower angular size and a lower amount of captured light can preclude the visibility of faint streams and asymmetric isophotes.

Atkinson et al. (2013) found that the occurrence of tidal features in red galaxies increases with the mass of the galaxy. We detected this just for streams, shells and disturbed isophotes (not tails). They did not investigate the correlation with environment density.

Pop et al. (2018) presented a census of shells in galaxies with masses over $10^{11} \mathrm{M}_{\odot}$ in a cosmological hydrodynamical simulation, finding the shell incidence of $20-30 \%$. This agrees well with our finding of $23 \pm 8 \%$ of at least likely detections in our sample when the same mass cut is applied. They however did not apply any limits on the surface brightness.

\subsubsection{Secular features}

We detected strong or weak secular features (bars, spiral arms, or rings but mostly bars) in $36 \pm 6 \%$ of galaxies. For comparison Krajnović et al. (2011) detected, in the very same sample (i.e. after excluding the $\operatorname{ATLAS}^{3 \mathrm{D}}$ galaxies that do not belong to the MATLAS sample), $28 \pm 4 \%$ galaxies with bars or rings but in images taken with $2.5 \mathrm{~m}$ optical telescopes.

Laurikainen et al. (2013) investigated the occurrence of bars in lenticular galaxies in a magnitude limited sam- 
ple using near-infrared ground-based images taken by $3-4 \mathrm{~m}$ class telescopes. They found that the bar occurrence depends on the Hubble stage number of the galaxy increasing from $\sim 35 \%$ for the -3 type to $\sim 75 \%$ for the -1 type. We calculated from their Table 1 that they found $57 \pm 6 \%$ of barred galaxies among the morphological types from -3 to 0 . To make a fair comparison, we obviously had to restrict ourselves to lenticular galaxies in our sample. Additionally, we wanted to minimize the effect of the different fraction of the morphological sub-classes of lenticular galaxies in the two samples. This led us to consider the Hubble stage number for our galaxies given in Cappellari et al. (2011a) and divide the galaxies into several bins centered on the integer stage numbers and having widths of one stage number. We multiplied the numbers of both barred and nonbarred galaxies in each bin by a constant so that the total number of galaxies in the bin was the same as in the corresponding bin of Laurikainen et al. (2013). The $57 \pm 6 \%$ of barred galaxies in the sample of Laurikainen et al. (2013) should be compared to $44 \pm 7 \%$ of barred galaxies in our sample. Our results are therefore consistent with Laurikainen et al. (2013). We counted as barred those of our galaxies having the rounded rating of at least 1 (i.e., loosely speaking, at least weak bars). The error for our sample was estimated in a Monte-Carlo way.

As for the correlations of bar occurrence with mass and environment density, Wilman \& Erwin (2012) divide galaxies to central and non-central with respect to their group. They found evidence that the bar incidence depends on the stellar mass of the galaxy only for the low-mass central galaxies. For them, the bar incidence is enhanced with respect to the level defined by the non-central galaxies. Barway et al. (2011) demonstrated that bar occurrence in lenticulars depends on mass and environment. They found that the bar fraction decreases with luminosity of the galaxy and that the bar fraction increases with the environment density. The dependence on the environment density is stronger for faint galaxies. Interestingly, bar occurrence does not depend on environment density for a general population of disk galaxies, i.e. consisting of both lenticular and spiral galaxies (Aguerri et al. 2009; Martínez \& Muriel 2011; Lin et al. 2014).

In order to make a comparison with these older works, we had to restrict ourselves only to the lenticular galaxies in our sample, i.e. to morphological types between -3.5 and 0.5. For such galaxies, the sample as a whole does not correlate significantly with $M_{\text {JAM }}$. The incidence of bars neither correlates with $\rho_{10}$, neither for the whole sample, nor with the low- or high-density halves. There is just a hint of correlation for the low-density half of the lenticular sample (density below $\left.\log _{10} \rho_{10} /\left(\mathrm{Mpc}^{-3}\right)=-1.63\right)$ - bar occurrence decreases with environment (Spearman coefficient of -0.2) at the $8 \%$ confidence level. We have to remind here again that our survey, unlike the previous works, excludes galaxy clusters, i.e. the densest environments. Similarly, the correlation of bar occurrence with the $M_{\mathrm{JAM}}$ mass is not significant for the total sample of our lenticulars. We however detect, contrarily to the literature results, that the bar occurrence increases with galaxy mass for the low-mass half of the sample of lenticulars (i.e., mass below $\log _{10} M_{\mathrm{JAM}} / M_{\odot}<10.56$ ) at a statistically significant confidence level of $4 \%$.

\subsubsection{Dust}

We can learn about the history of dust detection in elliptical galaxies from the review by Kormendy \& Djorgovski (1989). The authors say that many dust clouds in ellipticals are small and their detection depends critically on the resolving power of the instrument and seeing. Apart from this, the detection also requires dividing the image by a smooth model of the galaxy, which we did not do. This is perhaps the reason why we detected traces of dust only in $15 \pm 4 \%$ of our galaxies. Kormendy \& Djorgovski (1989) state a dust occurrence between 20 and $40 \%$.

Older works report an absence of correlation between dust mass and stellar mass or luminosity (e.g., Smith et al. 2012; Hirashita et al. 2015; Kokusho et al. 2019). In order to verify this in our sample, we restricted our analysis to elliptical galaxies, i.e. the morphological types below -3.5. There is indeed no significant correlation with $M_{\mathrm{JAM}}$ (the $p$ value of the Spearman coefficient is over the $5 \%$ threshold, even for the low- or high-mass halves).

\subsubsection{Peripheral disks}

We are not aware of any other precise statistics of the occurrence of peripheral star-forming disks in ETGs. A few ETGs in the MATLAS sample with evidence of peripheral star-forming disks and associated extended disk of atomic hydrogen have been studied in Yildiz et al. (2017). Galaxies with extended star formation (which is also visible in the UV survey by GALEX) actually resemble massive LSB galaxies such as Malin 1 (Galaz et al. 2015; Boissier et al. 2016). The latter consist of a faint disk, which is actually more extended than in our ETGs, and a prominent bulge. We note that our nearby ETGs with peripheral disks might be local analogs of galaxies at high redshifts: Sachdeva et al. (2019) suggested that disks frequently form around pre-existing bulges at the redshift of 2. Some of the peripheral disks might also possibly be analogs of polar rings appearing however in the equatorial plane of the galaxy.

\subsection{Interpreting the results}

Let us draw preliminary conclusions from our results, although an exhaustive analysis is beyond the scope of this paper. The incidences of structures have to be compared with the numbers predicted by theoretical models of galaxy formation. Here we thus focus just on tentative qualitative theoretical explanations for the observed trends of the incidences with the mass and environment of the target galaxy.

We detected statistically significant increase of the incidence of shells, streams and disturbed outer isophotes with the mass of the galaxy. We can think of several reasons for this. More massive galaxies have stronger tidal forces that can disrupt more massive neighbors and in a greater distance. The debris of bigger galaxies are also probably observable for a longer time. A more massive galaxy can attract its neighbors from larger distances and disrupt them afterward. Apart from this, more massive galaxies usually have a larger number of satellites and reside in denser environments such that there are more objects available for disruption. In a hierarchical model of galaxy formation, a higher abundance 


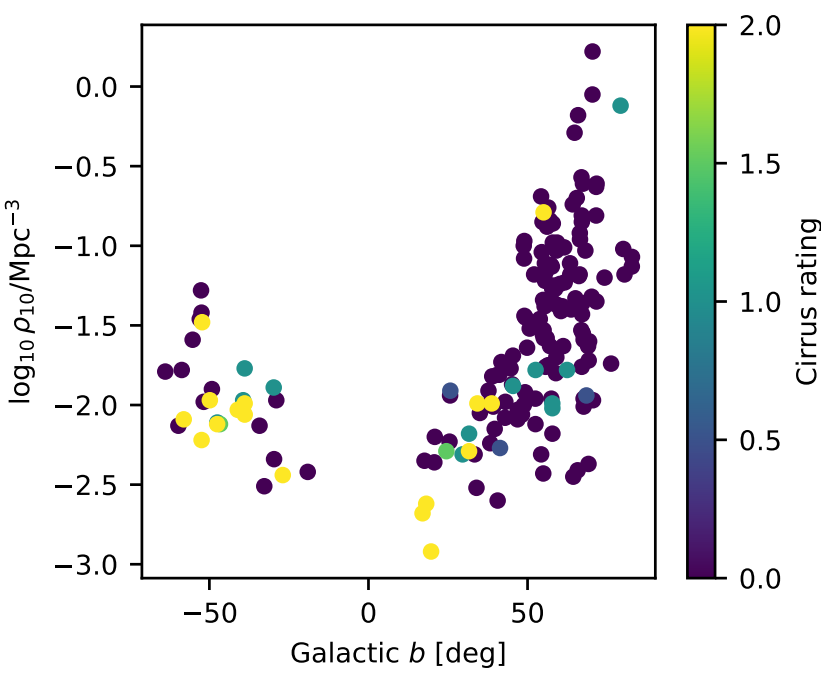

Figure 5. Environment densities of external galaxies depend on Galactic latitude.

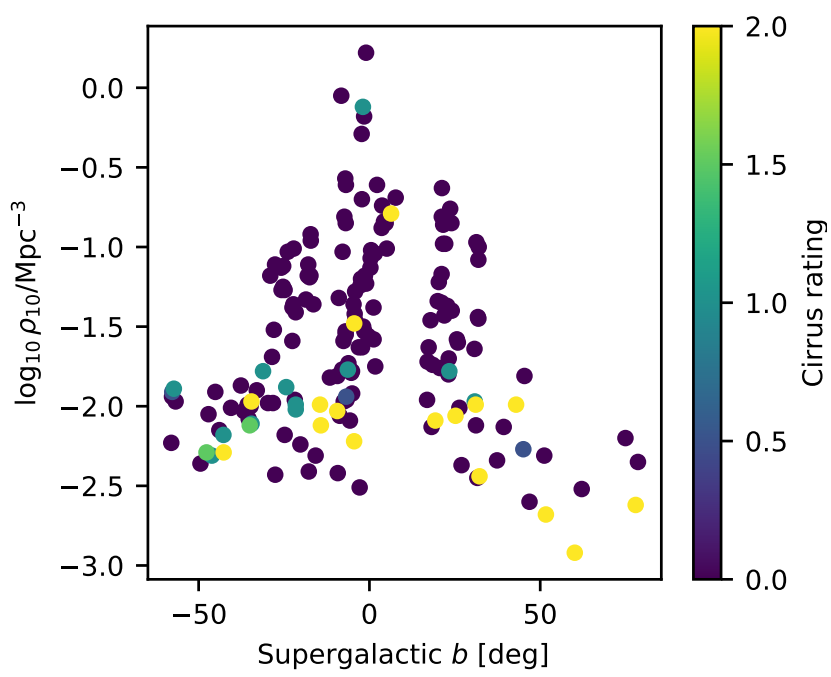

Figure 6. Environment densities of external galaxies depend on Supergalactic latitude.

of tidal features in massive galaxies is expected since more mass was accreted by these galaxies.

We found hints of correlations of the incidence of all types of tidal features with environment density. However, only two of them are statistically significant: the one for the incidence of tails and that for the incidence of shells in the low-density half of the sample. Indeed, we expect more frequent galaxy encounters in denser environments. The peak in the abundance of shells in the medium density bin is not statistically significant with the current data but there is a theoretical motivation for it. It is the result of two competing factors. On one hand, galaxy encounters are less likely in sparse environments. On the other hand, theoretical arguments suggest that shells form more likely if the accreted galaxy, that subsequently turns into the shells, is a disk galaxy (Hernquist \& Quinn 1988) and it is known that disk galaxies are less frequent in high density environ- ments. Moreover, stars released from the accreted galaxy can be so fast in the high-velocity encounters in the dense environments that they exceed the escape velocity, or dynamical friction during galaxy high-velocity encounters is ineffective. Many of the tails extend from galaxies that appear being disrupted by a more massive neighbor.

We found an anticorrelation of the incidence of peripheral star-forming disks with the environment density. It is in line with the well-known relation between the gas content of disk galaxies and environment - gas rich spiral galaxies are frequent in sparser environments while gas poor lenticulars are found rather in denser environments. One reason for this is ram pressure stripping of gas as the galaxy moves quickly through the intercluster/intergroup medium. If the ram pressure is not strong enough to remove the gas from the galaxy, star formation is quenched by the starvation mechanism when the surrounding hot intergroup medium prevents the accretion of the cold intergalactic medium that is available in the low density environment to feed the star formation in the galaxy. Star formation can also be quenched because of the gas is shocked and heated by galaxy interactions (Bitsakis et al. 2016; Ardila et al. 2018; Bitsakis et al. 2019).

Most surprisingly, the strongest correlation we found is that between the occurrence of Galactic cirrus and the environment of the background galaxy - the stronger cirrus, the lower environment density. We propose two possible explanations. One is based on the hypothesis that the environment density was underestimated at low galactic latitudes because of dust obscuration, as Fig. 5 suggests. The cirri are located close to the Galactic plane as well. There are however two arguments against this hypothesis. First, the incidence of Galactic cirrus does not correlate that well with a smallscale environment density $\Sigma_{3}$, defined as the mean projected density in a cylinder containing three nearest neighbors, a quantity from Cappellari et al. (2011b). The $p$-value of the correlation with $\Sigma_{3}$ is $0.4 \%$, while that of the correlation with $\rho_{10}$ is $10^{4}$ times lower. Besides, the environment density $\rho_{10}$ was determined from the $K$-band near-infrared images from the Two-Micron All-Sky Survey (Skrutskie et al. 2006). Even the $B$-band extinction for our galaxies listed in Cappellari et al. (2011a) is not high enough to explain the drop of environment density, when the Schechter function is taken into account, and this extinction is typically ten times greater than in the $K$-band.

We find it most probable that the trend is an indirect consequence of the orientation of the Milky Way disk with respect to the local structure of the cosmic web. It is well known that the Milky Way disk is nearly perpendicular to the Local Sheet, which defines the equatorial plane of the supergalactic coordinate system. The environment density indeed increases with the supergalactic latitude of the target galaxy, see Fig. 6.

\section{FUTURE PROSPECTS}

The visual classification method adopted in this paper allows us to distinguish the various types of tidal features, which give constraints on the mass assembly of the galaxy (e.g., minor/major or dry/wet mergers). Tidal features bring even information about the collision date because diffuse and 
sharp-edged tidal features have different survival times at out observation depth (Mancillas et al. 2019).

Obviously the main drawback of the visual method is its intrinsic subjectivity (see the discussion above) and its unfeasibility on very large samples. The forthcoming deep and wide-field surveys (LSST, CFIS, Euclid) create pressure to develop automated algorithms able to detect and classify tidal features in an enormous number of galaxies (see e.g., Laine et al. 2018). The use of automatic methods, for instance based on a deep learning (DL) approach, will become inevitable. However such algorithms will not necessarily allow us to overcome the subjective nature of the visual inspection, as DL requires to compile a training set, also relying on a classification by eye, unless numerical simulations are used. One major difficulty when trying to distinguish with DL different types of tidal features with subtle differences in their morphology, such as streams and tails, is the currently small sample of training images that had previously been annotated visually. Under such conditions, further developments are needed to guide the neural networks in the learning process.

Besides, one wishes to go beyond the qualitative methods which consists of just counting the number of fine features or estimating their strength. Getting quantitative requires doing the proper photometry of the collisional debris. This remains a challenge given their low surface brightness nature. A number of techniques are being developed to automatically trace faint features and obtain precise measurements of the sky background level (Akhlaghi \& Ichikawa 2015). However the level of fine tuning they require still prevents from applying them to large surveys, including MATLAS.

\section{SUMMARY}

In this paper we presented the first results from the MATLAS deep-optical-imaging volume-limited survey of 177 nearby ETGs that were drawn from the ATLAS ${ }^{3 \mathrm{D}}$ sample (Cappellari et al. 2011a) of well-explored galaxies. Our goal was to survey the incidence of the following structures in the galaxies: tidal streams, tails, and shells, the irregularity of outermost isophotes, bars in the centers of galaxies, dust lanes, presence of star formation at the outskirts of the galaxy, and the presence of close or interacting companions. Additionally, we investigated the incidence of two frequent pollutants of the images - polluting halos and galactic cirri. The detection and classification was performed visually by a group of researchers who had a substantial previous experience with the investigations of ETGs and interacting galaxies. The results presented here are based on votes of six individuals who inspected the over two-thirds of the whole sample. We found that the people who classified a low number of galaxies made less reliable classifications. This should be taken into account in future similar projects, especially those involving citizen science. The structures identified for each galaxy are summarized in Table D1. The statistics of the incidence of the features are presented in Table 5. We then investigated the correlation of the incidences of the structures of interest with the mass of the target galaxy and its environment density. The main result is Table 6 that gives the Spearman rank coefficient of the correlation along with its $p$-value. We compared our results with older publications in Sect. 5.2. We found an extremely strong unexpected anticorrelation of the environment density with the occurrence of the foreground pollution by Galactic cirrus ( $p$-value of $3 \times 10^{-5 \%}$ ), a positive correlation of the mass of the galaxy with the presence of tidal streams, shells and the irregularity of outer isophotes; the correlation with the occurrence tidal tails is not statistically significant. We found a statistically significant anticorrelation between the environmental density of the galaxy and the presence of a peripheral star forming disk. A qualitative interpretation of the results is provided in Sect. 5.3. Briefly, we suggests that the correlation of environment density and cirrus incidence is due to the perpendicular orientation of the Milky Way disk plane with respect to the local structure of the cosmic web. More massive galaxies contain more tidal features and disturbances because of their stronger gravitational attraction and stronger tidal forces. In the future, we plan to look for the correlations of the incidence of the morphological structures presented here with the many other parameters available for the ATLAS ${ }^{3 \mathrm{D}}$ galaxies. We also develop software that would substitute the visual detection and classification of morphology in the future large surveys.

\section{ACKNOWLEDGEMENTS}

We thank Dr. Ivana Ebrová for valuable comments. S.P. acknowledges support from the New Researcher Program (Shinjin grant No. 2019R1C1C1009600) through the National Research Foundation of Korea. 


\section{REFERENCES}

Abazajian K. N., et al., 2009, ApJS, 182, 543

Abraham R. G., Tanvir N. R., Santiago B. X., Ellis R. S., Glazebrook K., van den Bergh S., 1996, MNRAS, 279, L47

Adams S. M., Zaritsky D., Sand D. J., Graham M. L., Bildfell C., Hoekstra H., Pritchet C., 2012, AJ, 144, 128

Aguerri J. A. L., Méndez-Abreu J., Corsini E. M., 2009, A\&A, 495,491

Akhlaghi M., Ichikawa T., 2015, ApJS, 220, 1

Alabi A., et al., 2018, MNRAS, 479, 3308

Alabi A. B., Forbes D. A., Romanowsky A. J., Brodie J. P., 2020, MNRAS, 491, 5693

Amorisco N. C., Loeb A., 2016, MNRAS, 459, L51

Annibali F., et al., 2020, MNRAS, 491, 5101

Ardila F., et al., 2018, ApJ, 863, 28

Atkinson A. M., Abraham R. G., Ferguson A. M. N., 2013, ApJ, 765,28

Banik I., Zhao H., 2018, MNRAS, 473, 4033

Barway S., Wadadekar Y., Kembhavi A. K., 2011, MNRAS, 410, L18

Bennet P., Sand D. J., Zaritsky D., Crnojević D., Spekkens K., Karunakaran A., 2018, ApJ, 866, L11

Bertin E., Pillay R., Marmo C., 2015, Astronomy and Computing, 10, 43

Bílek M., Jungwiert B., Jílková L., Ebrová I., Bartošková K., Křížek M., 2013, A\&A, 559, A110

Bílek M., Ebrová I., Jungwiert B., Jílková L., Bartošková K., 2015a, Canadian Journal of Physics, 93, 203

Bílek M., Jungwiert B., Ebrová I., Bartošková K., 2015b, A\&A, 575, A29

Bílek M., Thies I., Kroupa P., Famaey B., 2018, Astronomy and Astrophysics, 614, A59

Bílek M., Thies I., Kroupa P., Famaey B., 2019a, arXiv e-prints, p. arXiv:1908.07537

Bílek M., Samurović S., Renaud F., 2019b, A\&A, 625, A32

Bitsakis T., et al., 2016, MNRAS, 459, 957

Bitsakis T., et al., 2019, MNRAS, 483, 370

Boissier S., et al., 2016, Astronomy and Astrophysics, 593, A126

Bridge C. R., Carlberg R. G., Sullivan M., 2010, ApJ, 709, 1067

Bullock J. S., Johnston K. V., 2005, ApJ, 635, 931

Byun W., et al., 2018, AJ, 156, 249

Capaccioli M., et al., 2015, A\&A, 581, A10

Cappellari M., 2008, MNRAS, 390, 71

Cappellari M., et al., 2011a, MNRAS, 413, 813

Cappellari M., et al., 2011b, MNRAS, 416, 1680

Cappellari M., et al., 2013, MNRAS, 432, 1709

Carleton T., Errani R., Cooper M., Kaplinghat M., Peñarrubia J., Guo Y., 2019, MNRAS, 485, 382

Chan T. K., Kereš D., Wetzel A., Hopkins P. F., Faucher-Giguère C. A., El-Badry K., Garrison-Kimmel S., Boylan-Kolchin M., 2018, MNRAS, 478, 906

Conselice C. J., 2003, ApJS, 147, 1

Cooper A. P., et al., 2010, MNRAS, 406, 744

Dabringhausen J., Kroupa P., 2013, MNRAS, 429, 1858

Dalcanton J. J., Spergel D. N., Gunn J. E., Schmidt M., Schneider D. P., 1997, AJ, 114, 635

Danieli S., et al., 2019, arXiv e-prints, p. arXiv:1910.14045

Di Cintio A., Brook C. B., Dutton A. A., Macciò A. V., Obreja A., Dekel A., 2017, MNRAS, 466, L1

Draper A. R., Ballantyne D. R., 2012, ApJ, 753, L37

Duc P.-A., et al., 2015, MNRAS, 446, 120

Ebrová I., Jílková L., Jungwiert B., Kř́ižek M., Bílek M., Bartošková K., Skalická T., Stoklasová I., 2012, A\&A, 545, A33

Ebrová I., Bílek M., Yıldız M. K., Eliášek J., 2020, A\&A, 634, A73

Eigenthaler P., et al., 2018, ApJ, 855, 142

Ferrarese L., et al., 2012, ApJS, 200, 4
Fliri J., Trujillo I., 2016, MNRAS, 456, 1359

Forbes D. A., Read J. I., Gieles M., Collins M. L. M., 2018, MNRAS, 481, 5592

Galaz G., Milovic C., Suc V., Busta L., Lizana G., Infante L., Royo S., 2015, The Astrophysical Journal, 815, L29

George K., 2017, A\&A, 598, A45

Gilhuly C., et al., 2019, arXiv e-prints, p. arXiv:1910.05358

González N. M., Smith Castelli A. V., Faifer F. R., Escudero C. G., Cellone S. A., 2018, A\&A, 620, A166

Greco J. P., et al., 2018, ApJ, 857, 104

Habas R., et al., 2019, arXiv e-prints, p. arXiv:1910.13462

Harris W. E., Harris G. L., Hudson M. J., 2015, ApJ, 806, 36

Hendel D., Johnston K. V., 2015, MNRAS, 454, 2472

Hendel D., Johnston K. V., Patra R. K., Sen B., 2019, MNRAS, 486, 3604

Hernquist L., Quinn P. J., 1988, ApJ, 331, 682

Hirashita H., Nozawa T., Villaume A., Srinivasan S., 2015, MNRAS, 454, 1620

Hood C. E., Kannappan S. J., Stark D. V., Dell'Antonio I. P., Moffett A. J., Eckert K. D., Norris M. A., Hendel D., 2018, ApJ, 857, 144

Impey C., Bothun G., Malin D., 1988, ApJ, 330, 634

Iodice E., et al., 2016, ApJ, 820, 42

Iodice E., et al., 2017a, ApJ, 839, 21

Iodice E., et al., 2017b, ApJ, 851, 75

Iodice E., et al., 2020, A\&A, 635, A3

Janssens S. R., Abraham R., Brodie J., Forbes D. A., Romanowsky A. J., 2019, ApJ, 887, 92

Javanmardi B., Kroupa P., 2020, MNRAS, 493, L44

Javanmardi B., et al., 2016, A\&A, 588, A89

Johnston K. V., Bullock J. S., Sharma S., Font A., Robertson B. E., Leitner S. N., 2008, ApJ, 689, 936

Kado-Fong E., et al., 2018, ApJ, 866, 103

Karabal E., Duc P. A., Kuntschner H., Chanial P., Cuillandre J. C., Gwyn S., 2017, Astronomy and Astrophysics, 601, A86 Kaviraj S., 2010, MNRAS, 406, 382

Koda J., Yagi M., Yamanoi H., Komiyama Y., 2015, ApJ, 807, L2

Kokusho T., et al., 2019, A\&A, 622, A87

Kormendy J., Djorgovski S., 1989, ARA\&A, 27, 235

Krajnović D., et al., 2011, MNRAS, 414, 2923

Kroupa P., Theis C., Boily C. M., 2005, A\&A, 431, 517

Laine S., et al., 2016, AJ, 152, 72

Laine S., et al., 2018, arXiv e-prints, p. arXiv:1812.04897

Laurikainen E., Salo H., Athanassoula E., Bosma A., Buta R., Janz J., 2013, MNRAS, 430, 3489

Liao S., et al., 2019, MNRAS, 490, 5182

Libeskind N. I., Guo Q., Tempel E., Ibata R., 2016, ApJ, 830, 121

Lim S., et al., 2017, ApJ, 835, 123

Lin Y., Cervantes Sodi B., Li C., Wang L., Wang E., 2014, ApJ, 796, 98

Lintott C. J., et al., 2008, MNRAS, 389, 1179

Lotz J. M., Primack J., Madau P., 2004, AJ, 128, 163

Malhan K., Ibata R. A., 2019, MNRAS, 486, 2995

Malin D. F., Carter D., 1983, ApJ, 274, 534

Mancillas B., Duc P. A., Combes F., Bournaud F., Emsellem E., Martig M., Michel-Dansac L., 2019, arXiv e-prints, p. arXiv:1909.07500

Martínez H. J., Muriel H., 2011, MNRAS, 418, L148

Martínez-Delgado D., 2019, in Montesinos B., Asensio Ramos A., Buitrago F., Schödel R., Villaver E., Pérez-Hoyos S., OrdóñezEtxeberria I., eds, Highlights on Spanish Astrophysics X. pp 146-154 (arXiv:1811.12286)

Martínez-Delgado D., Peñarrubia J., Gabany R. J., Trujillo I., Majewski S. R., Pohlen M., 2008, ApJ, 689, 184

Martínez-Delgado D., Pohlen M., Gabany R. J., Majewski S. R., Peñarrubia J., Palma C., 2009, ApJ, 692, 955 
McIntosh D. H., Guo Y., Hertzberg J., Katz N., Mo H. J., van den Bosch F. C., Yang X., 2008, MNRAS, 388, 1537

Merritt A., van Dokkum P., Abraham R., Zhang J., 2016, ApJ, 830,62

Mihos J. C., et al., 2015, ApJ, 809, L21

Mihos J. C., Harding P., Feldmeier J. J., Rudick C., Janowiecki S., Morrison H., Slater C., Watkins A., 2017, ApJ, 834, 16

Mihos J. C., Carr C. T., Watkins A. E., Oosterloo T., Harding P., 2018, ApJ, 863, L7

Miskolczi A., Bomans D. J., Dettmar R. J., 2011, A\&A, 536, A66

Miville-Deschênes M. A., Duc P. A., Marleau F., Cuillandre J. C., Didelon P., Gwyn S., Karabal E., 2016, A\&A, 593, A4

Morales G., Martínez-Delgado D., Grebel E. K., Cooper A. P., Javanmardi B., Miskolczi A., 2018, A\&A, 614, A143

Muñoz R. P., et al., 2015, ApJ, 813, L15

Müller O., Jerjen H., Pawlowski M. S., Binggeli B., 2016, A\&A, 595, A119

Müller O., et al., 2019a, A\&A, 624, L6

Müller O., Rejkuba M., Pawlowski M. S., Ibata R., Lelli F., Hilker M., Jerjen H., 2019b, A\&A, 629, A18

Müller O., Vudragović A., Bílek M., 2019c, A\&A, 632, L13

Nair P. B., Abraham R. G., 2010, ApJS, 186, 427

Oh S., et al., 2019, MNRAS, 488, 4169

Papastergis E., Adams E. A. K., Romanowsky A. J., 2017, A\&A, 601, L10

Pawlik M. M., Wild V., Walcher C. J., Johansson P. H., Villforth C., Rowlands K., Mendez-Abreu J., Hewlett T., 2016, MNRAS, 456, 3032

Pawlowski M. S., Bullock J. S., Kelley T., Famaey B., 2019, ApJ, 875,105

Pearson W. J., Wang L., Trayford J. W., Petrillo C. E., van der Tak F. F. S., 2019, A\&A, 626, A49

Peng C. Y., Ho L. C., Impey C. D., Rix H.-W., 2002, AJ, 124, 266

Peters S. P. C., van der Kruit P. C., Knapen J. H., Trujillo I., Fliri J., Cisternas M., Kelvin L. S., 2017, MNRAS, 470, 427

Poci A., Cappellari M., McDermid R. M., 2017, MNRAS, 467, 1397

Pop A.-R., Pillepich A., Amorisco N. C., Hernquist L., 2018, MNRAS, 480, 1715

Quinn P. J., 1983, in Athanassoula E., ed., IAU Symposium Vol. 100, Internal Kinematics and Dynamics of Galaxies. p. 347

Rich R. M., et al., 2019, MNRAS, p. 2031

Román J., Trujillo I., 2017a, MNRAS, 468, 703

Román J., Trujillo I., 2017b, MNRAS, 468, 4039

Román J., Trujillo I., Montes M., 2019, arXiv e-prints, p. arXiv:1907.00978

Sachdeva S., Gogoi R., Saha K., Kembhavi A., Raychaudhury S., 2019, MNRAS, 487, 1795

Samurović S., 2014, A\&A, 570, A132

Sandage A., Binggeli B., 1984, AJ, 89, 919

Sanders D. B., Mirabel I. F., 1996, ARA\&A, 34, 749

Sanderson R. E., Helmi A., 2013, MNRAS, 435, 378

Sanderson R. E., Hartke J., Helmi A., 2017, ApJ, 836, 234

Sheen Y.-K., Yi S. K., Ree C. H., Lee J., 2012, ApJS, 202, 8

Skrutskie M. F., et al., 2006, AJ, 131, 1163

Smith M. W. L., et al., 2012, ApJ, 748, 123

Tal T., van Dokkum P. G., Nelan J., Bezanson R., 2009, AJ, 138, 1417

Thomas G. F., Famaey B., Ibata R., Lüghausen F., Kroupa P., 2017, A\&A, 603, A65

Thomas G. F., Famaey B., Ibata R., Renaud F., Martin N. F., Kroupa P., 2018, A\&A, 609, A44

Torrealba G., et al., 2019, MNRAS, 488, 2743

Trujillo I., Bakos J., 2013, MNRAS, 431, 1121

Trujillo I., Fliri J., 2016, ApJ, 823, 123

Walmsley M., Ferguson A. M. N., Mann R. G., Lintott C. J., 2019, MNRAS, 483, 2968
Wilman D. J., Erwin P., 2012, ApJ, 746, 160

Wright E. L., 2006, PASP, 118, 1711

Yıldız M. K., Serra P., Peletier R. F., Oosterloo T. A., Duc P.-A., 2017, MNRAS, 464, 329

Yıldız M. K., Peletier R. F., Duc P. A., Serra P., 2020, arXiv e-prints, p. arXiv:2001.08087

van Dokkum P. G., 2005, AJ, 130, 2647

van Dokkum P. G., Abraham R., Merritt A., Zhang J., Geha M., Conroy C., 2015, ApJ, 798, L45

van Dokkum P., et al., 2018a, Nature, 555, 629

van Dokkum P., et al., 2018b, ApJ, 856, L30

van Dokkum P., et al., 2019, ApJ, 883, L32

\section{APPENDIX A: METHODS}

The MATLAS images were inspected visually. The participants were provided a web interface with a navigation tool to display the image and a questionnaire to fill in. Initially, participants received a brief training and instructed how to use the web interface, classify the structures of interest and fill the questionnaire. The participants were not required to inspect all galaxies. They could change their answers later, e.g. when they gained more experience. Each participant filled out the questionnaire independently of the others. A bestguess strategy was adopted: the participants always chose the most probable option (in contrast to rejecting a null hypothesis) and, if the classified feature did not fit to any of the offered categories exactly, they chose the closest option (e.g., when the feature appeared as an unusual shell) or the most probable option (e.g., because the faintness of the feature made the classification difficult).

\section{A1 Participants}

There were 15 participants in total. The results we present in this paper are however based just on the votes of six participants who inspected more than two-thirds of the sample. The other participants inspected usually much less galaxies. As we show in Appendix B, the votes of the six experienced participants agree with each other better than if the votes of all participants are considered. A histogram of the number of votes per galaxy that we used is shown in Fig. A1; the median number of votes per galaxy is six and every galaxy was inspected at least twice. We used 861 votes.

\section{A2 Navigation tool}

The navigation tool (Fig. A2) used for the classification is based on the VisiOmatic web client (Bertin et al. 2015) which is based on the Leaflet Javascript library and the IIPImage server. The tool allowed us to visualize and navigate and zoom in/out through large science images from remote locations. It was customized for the need of the project.

The navigation tool allowed us to inspect the data in many ways. After opening the web page with the tool (Fig. A2), a composite RGB image was shown. The RGB image planes were arranged such that the Red channel contained the $i^{\prime}$ band data, Green the $r^{\prime}$ band data, and Blue the $g^{\prime}$ band data, when the 3 bands are available. If images in only two photometric bands were available, namely $g^{\prime}$ and $r^{\prime}$, the Green plane was an average of them. To enhance the low surface brightness features, an arcsinh intensity scaling 


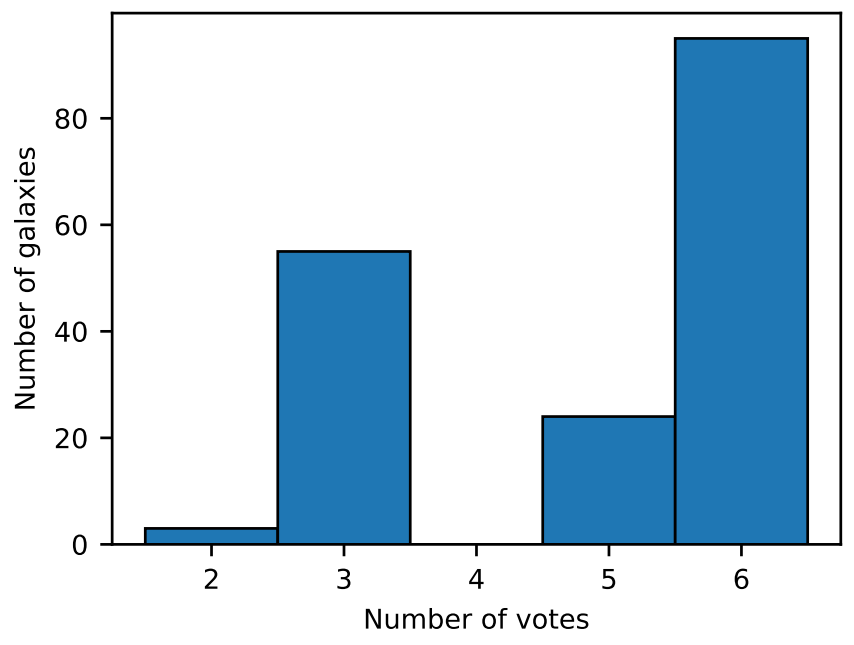

Figure A1. Histogram of the number of votes per galaxy. The median number of votes per galaxy is six.

was applied. Additional parameters could be set to adjust the nonlinear intensity scaling of the image. The choice of the linear scaling led for many galaxies to posterization of the image which could have precluded us from detecting some structures. There were also available color index maps that were particularly useful for detecting dust patches and regions of recent star formation. Mono-band surface brightness maps scaled in mag arcsec ${ }^{-2}$ could also be selected. All images could be zoomed, panned or changed intensity and color saturation levels. We found that the features of interest were visible best if we inspected a grayscale image of only one of the available bands and adjusted the contrast. On the contrary, seeing a faint structure in several photometric bands allowed us to confirm that the object was real. The color images were particular useful when identifying the reflections around bright objects that are the brightest in the $r^{\prime}$ band filter and appeared as green in the RGB images. The images reached the best signal-to-noise ratio in the $g^{\prime}$ band while the $i^{\prime}$ band images suffered the least of the parasitic reflections. The visibility of very faint structures depended on the magnification of the image. A tool was available to display the light/color profiles in two directions along a line. For some galaxies, there were available residual images obtained by subtraction of the smooth model of the galaxy constructed by Galfit (Peng et al. 2002).

Finally, further information on the properties of the galaxies in the ATLAS $^{3 \mathrm{D}}$ catalog, including massive latetype galaxies from the parent sample, was provided in displayed labels. These included magnitude, distance and radial velocity. Furthermore, objects in the field with available SDSS data could be visualized.

All the on-line images and the navigation tool that were used in this study is made publicly available at http: //obas-matlas.u-strasbg.fr/. Examples can be seen in Appendix C.

\section{A3 Questionnaire}

Here we give a more detailed description of the questionnaire.
- Participants were first asked about the presence of shells, streams or tails around the galaxy. For each type of structure, the available answers were: No, Likely, Yes, and Unsure. The number of features for every category had to be indicated. The structures classified here as tails are defined as elongated features directly attached to the target galaxy and that appear consistent with having been formed in an ongoing interaction or a recent merger. The stellar mass of the companion in the interaction has to be similar to or even higher than that of the target galaxy. Depending on the morphology of the interacting partners and encounter stage, a tail could appear as an antennae-like structure similar to the ones observed in the prototypical advanced merger NGC 7252 ("The Atoms for Peace") - the merged companion was a spiral galaxy - or a plume-like structure with a thickness comparable to the size of the body of the target galaxy - the accreted massive companion was an elliptical galaxy or the target ETG is currently interacting with the still existing massive companion.

The structures classified here as streams are elongated morphological structures which appear consistent with accreting a companion of a lower mass. They are usually thinner than the tails and can be wrapped around the galaxy. Some of them are detached from the target galaxy. They sometimes contain an embedded candidate for the surviving core of the disrupted galaxy.

Shells are arc-like sharp-edged features whose centers coincide with the center of the host galaxy. They are usually interpreted as results of radial mergers. The life-times of streams, tails and shells were estimated in Mancillas et al. (2019). We note that dominant formation mechanisms of these structures might be different in the context of modified gravity, where a large fraction of them is expected to arise from non-merging galaxy flybys (Bílek et al. 2018, 2019a).

- The next question was about the shape of the outermost detectable isophote. The available answers were: Regular, Asymmetric, Disturbed, or Unsure. By outermost isophote, we refer to the one which by eye seems unaffected by any artefacts or background fluctuations, and is therefore considered as reliable. Its level may thus vary from one field to the other and range between about 27 and $29 \mathrm{mag} \operatorname{arcsec}^{-2}$ in $g^{\prime}$. However, the asymmetries were generally visible even on the brighter isophotes. The Regular type of the outer isophotes refers to isophotes that are characterized well by an ellipse possibly with a boxy or disky modulation. The Asymmetric type showed only mild deviations form the regular shape possibly forming an S-shape (a warp), an ovoidal shape, or the ellipse contained a single mild bump. Most of the asymmetric isophotes might have been produced by distant or old interactions. On the contrary, the Disturbed isophotes are characterized by a complex shape signifying a recent or strong interaction. We note that if the galaxy contained a very large number of streams or shells, they could not be recognized individually but would instead appear as a blended irregular halo. The most frequent reason for classifying the shape of the outermost isophote as Unsure were either a poor background subtraction caused by the presence of a nearby bright object or that the outermost isophotes overlapped with another large nearby galaxy.

- The presence of dust was rated on the scale: No, Weak, Strong and Unsure. Dust appears in true color images as darker spots or lanes overlaid over the stellar light of the 


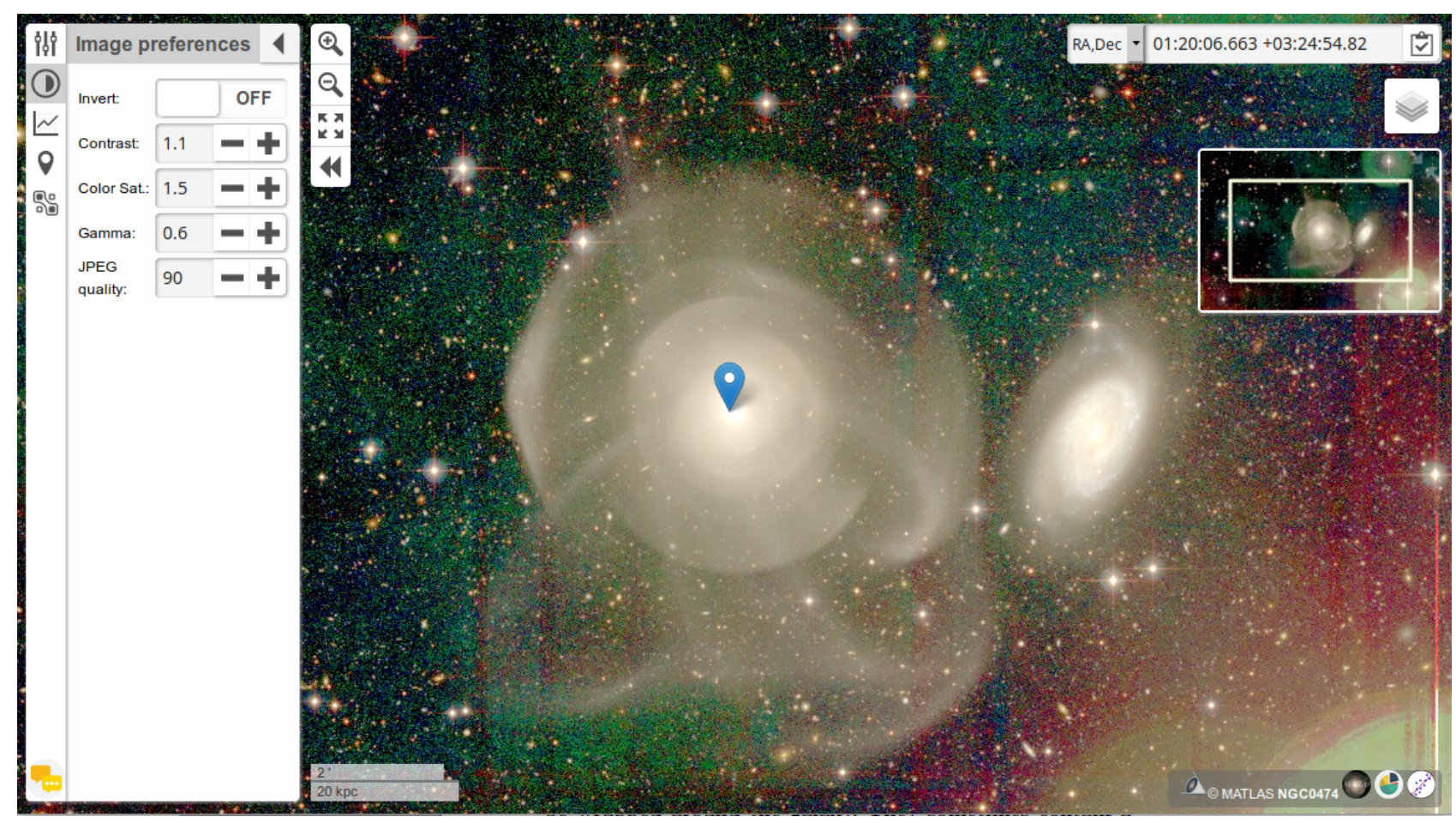

Figure A2. Navigation tool used for the classifications, here displaying the galaxies NGC 474 (left) and NGC 470 (right). Accessible at http://obas-matlas.u-strasbg.fr.

galaxy. Dust lanes were usually found in the centers of galaxies, but the depth of the images allowed us to identify them even further out. We might have missed the dust lanes at galaxy centers in the case of galaxies that have saturated centers in our deep images. In a few cases, the logged dust lanes were perhaps not in the target galaxy but they were parts of tidal features of a companion galaxy that were overlaid.

- The presence of a peripheral disk around the galaxy could be described with one of the following answers: No, Yes or Unsure. These disks of stars appear noticeably bluer than the red centers of the galaxies. They can form spiral arms and/or have a clumpy structure. In some cases, the star forming regions appear to have an external origin, being captured from a spiral galaxy companion. Because of their faintness, it is unclear whether the peripheral disks extend to the centers of the galaxies. The name "peripheral" means that they are best observed at the outskirts of the galaxies, beyond the main old stellar halo.

- The presence of features usually induced by secular evolution, i.e. internal bars, rings, or spiral arms (with no star formation) could be rated as No, Weak, Strong and Unsure. For the galaxies observed edge-on, the presence of an $\mathrm{X}$-structure was the criterion to estimate the presence of a bar. Saturated regions in the center might have hidden weak bars. We note that bars can also form during galaxy interactions.

- The presence of features disturbing the classification had then to be commented, starting with the presence of Galactic cirrus. Their presence and strength were evaluated as either No, Weak, Strong or Unsure. Cirri are identified as filamentary structures which usually come in groups, forming parallel bands or stripes. Cirri superimposed on the in- spected galaxy could possibly be confused with tidal debris. We annotated also the cirri being further out from the target galaxy but likely affecting the background subtraction decreasing the visibility of the faint structures indirectly. The large field of view of MegaCam allows us to directly identify cirri in the optical images without having to look for infrared images.

\section{A4 Summarizing the answers, numerical ratings}

Since every question was answered several times for every galaxy, once per participant, it was necessary to summarize all the answers into one final one. We call the result the rating. In order to define it, we first assigned numerical values to the answers. For tails, steams, shells, the answers [No; Likely; Yes; Unsure] were respectively converted to [0; $1 ; 2 ;-1]$; similarly for outer isophotes the answers [Regular; Asymmetric; Disturbed; Unsure] were converted to $[0 ; 1 ; 2$; -1]; and for dust lanes, bars and cirrus [No; Weak; Strong; Unsure $]$ to $[0 ; 1 ; 2 ;-1]$; finally for polluting halos or peripheral disks, the answers [No; Yes; Unsure] were respectively converted to $[0 ; 1 ;-1]$. Then we excluded for every galaxy the -1 answers motivated by our best-guess strategy. The rating of the presence of the given feature in the galaxy was defined as the answer that got most votes. In the "draw case" that several answers got the same highest number of votes, the result was the average of their numeric values: for example, if the votes were 2 for "Yes", 2 for "Likely" and 1 for "No", the result was $(2 \times 2+2 \times 1) / 4=1.5$. If all answers were -1 , then the final rating was -1 . When evaluating the polluting halos, the target galaxies whose isophotes overlap with another substantial neighbor were rated as 1 directly without voting, since there is rarely doubt about about such cases. Two of 
us identified such galaxies together. It was also necessary to decide about the number of streams, tails and shells in every galaxy. This was done in a similar way. We first chose the most frequent answers on presence of the feature from the set [Yes, Likely, No]. Then we worked just with votes of the participants who voted for the most frequent answers: for example, if there were 2 votes for "Yes", 2 for "Likely" and 1 for "No", we worked just with the votes of the participants who voted "Yes" or "Likely". The resulting number of the features was then calculated as the weighed average of the numbers given by the selected participants; the weights were either $[1 ; 0.5 ; 0]$ for the presence vote [Yes, Likely, No], respectively. If all answers were "Unknown" then the result was -1 .

We also used the "rounded rating". Here, we rounded the continuous rating described in the previous paragraph to the nearest integer (e.g., the rating of the presence of streams of 0.3 was rounded to 0 ). In the case of tidal features, bars, dust lanes and cirrus, the border values of 0.5 and 1.5 were rounded respectively to 0 or 2 in order to minimize the number of objects sorted in the intermediate category. In the case of polluting halos and peripheral disks, a continuous rating of 0.5 was rounded to 1 .

Our classifications could potentially have been affected by the so-called halos. We thus had to identify the galaxies that are affected by halos in order to assess their influence later. The first type of halos are ghost reflections, instrumental artifacts, appearing around bright stars, see, e.g., Figures C7-C9. They can be recognized easily because all bright stars in the image show a reflection an all reflections have the same size and very similar shapes. The reflections have been described in detail in Karabal et al. (2017). The second type of polluting halos are other galaxies close to the galaxy in question. Even they can be overlaid over tidal features a prevent their detection. We therefore considered the neighbors, whose isophotes overlap with the isophotes of the targets, as polluting halos. We logged only the halos, of either type, that could have plausibly caused a misclassification of the galaxy of interest. The polluting halos were identified by two people working together. Identifying polluting halos is generally easier and less subjective than, e.g., identifying tidal features. If a galaxy was polluted by a halo, it was assigned a numerical rating of halos of 1 , and 0 in the opposite case.

\section{APPENDIX B: THE EFFECT OF THE EXPERIENCE OF THE PARTICIPANTS}

In Figure B1 we compared the adopted rating to the rating based only on the votes of the less experienced participants , i.e. those who inspected less than two thirds of the sample. We examined the cases where the two ratings differed completely, i.e. where one came out to have the maximum value while the other came out 0 . In most cases the difference in rating can be accounted to classifying a well-visible feature in another category. The less experienced classifiers also had difficulties with detecting low-contrast features in a few cases. Figure B2 presents an example of a galaxy where shells were detected according to the standard rating but were not according to the rating by the less experienced group. The shells were likely missed because the galaxy with
Table B1. Comparison of the scatter in the in the default rating, based on the votes of the participants who inspected over twothirds of all galaxies, and the rating based on the votes of all participants.

\begin{tabular}{lll}
\hline \hline Structure & $\begin{array}{l}\text { More experienced } \\
\text { voters only }\end{array}$ & All voters \\
\hline Shells & 0.23 & 0.25 \\
Streams & 0.32 & 0.35 \\
Tails & 0.18 & 0.23 \\
Outer isophotes & 0.43 & 0.43 \\
Peripheral disks & 0.087 & 0.092 \\
Secular features & 0.45 & 0.45 \\
Dust & 0.25 & 0.27 \\
Cirrus & 0.37 & 0.38 \\
Halos & 0.27 & 0.27 \\
\hline
\end{tabular}

shells, NGC 3605, is seen overlaid over the body of a bright and large neighbor, NGC 3607, and therefore the contrast of the shells is reduced. The right panel of the figure shows that the contrast can be set in the navigator tool such that the shells become clearly visible. Those participants who have had inspected just a few galaxies before this one might have also confused the two neighboring galaxies.

We noted in Figure B1 the following: 1) The more experienced classifiers were more positive about the presence of shells, streams, disturbed isophotes, bars and cirrus. This is likely because the less experienced contributors missed the low-contrast features. 2) The less experienced contributors were more positive about the presence of tidal tails probably because of the confusion with streams. 3) The two groups of participants agree well about the presence of dust lanes, a feature easily detectable. 4) The less experienced voters were more positive about the presence of peripheral disks. The confusion happened in this case usually because of disturbing reflections of nearby bright stars or because of a change of color of the galaxy due to the PSF effects. 5) The two groups agree well when there is no feature or when the feature is prominent.

We further explored whether excluding the less experienced participants leads to more consistent results. For each galaxy and each feature class we calculated the standard deviation of the votes (after the conversion to a numerical value) on the presence of the feature within the group of the more experienced voters and within the group of all participants together. Then we calculated the result for each feature type as a weighed average of the scatters for individual galaxies, while the weights were the number of votes for the given galaxy. The comparison of the average scatters for the given feature for the group of experienced participants and the group of all contributors is presented in Table B1. The scatter between the two groups is mostly lower for the experienced participants or it is the same. We note that 59 galaxies, i.e. $33 \%$ of the sample, had votes only by the more experienced voters. Finally, we present in Table B2 the census based on the votes of all participants. One can note by comparison with the main census Table 5 that the two are consistent.

\section{APPENDIX C: EXAMPLES OF THE CLASSIFIED STRUCTURES}



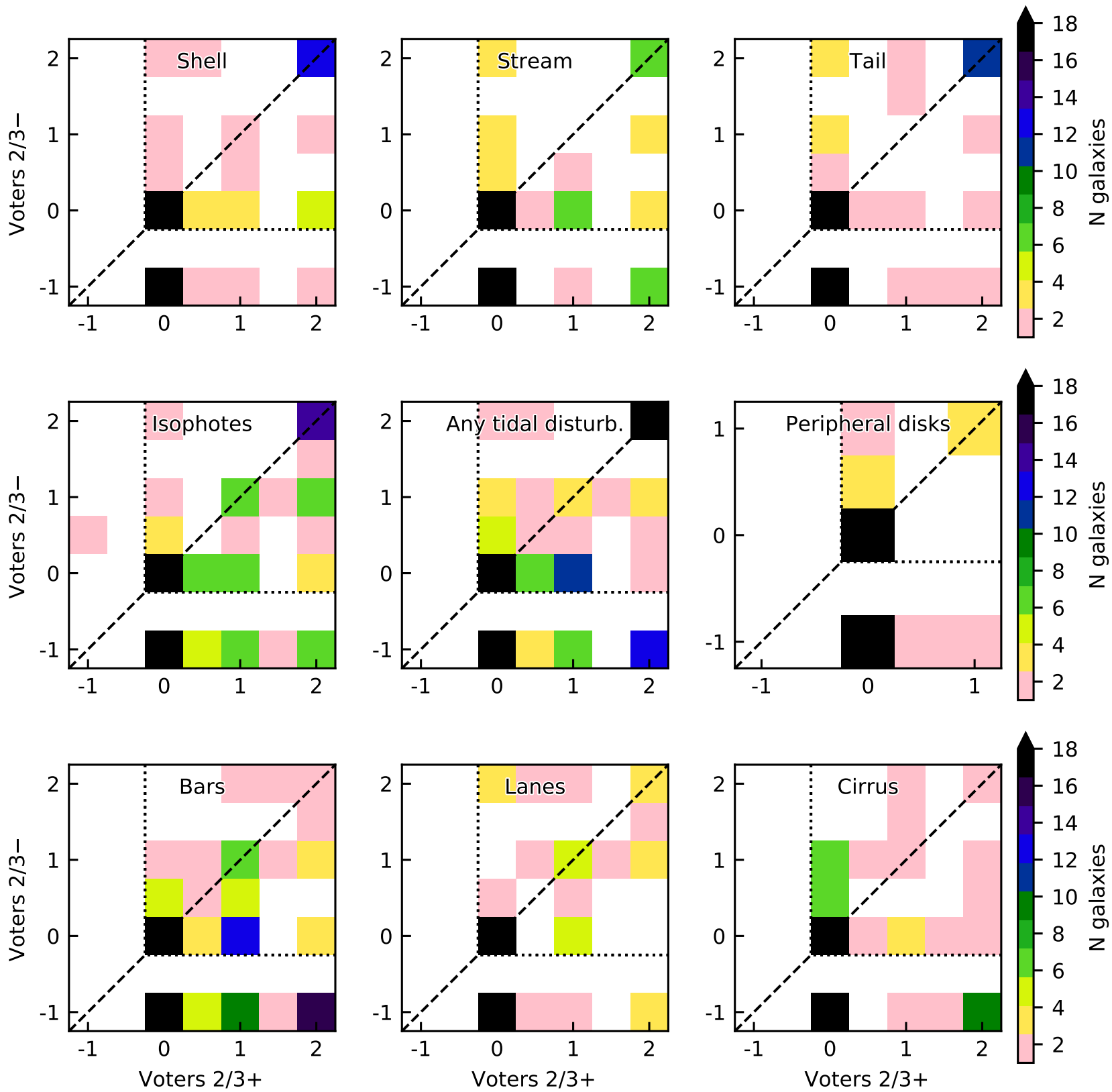

Figure B1. Comparison of the ratings based on the votes of the participants who inspected over two-thirds of galaxies (horizontal axis), and the ratings based on the votes of the participants who inspected a lower number of galaxies (vertical axis). The ratings of -1 mostly mean that there are no votes for the galaxy. 


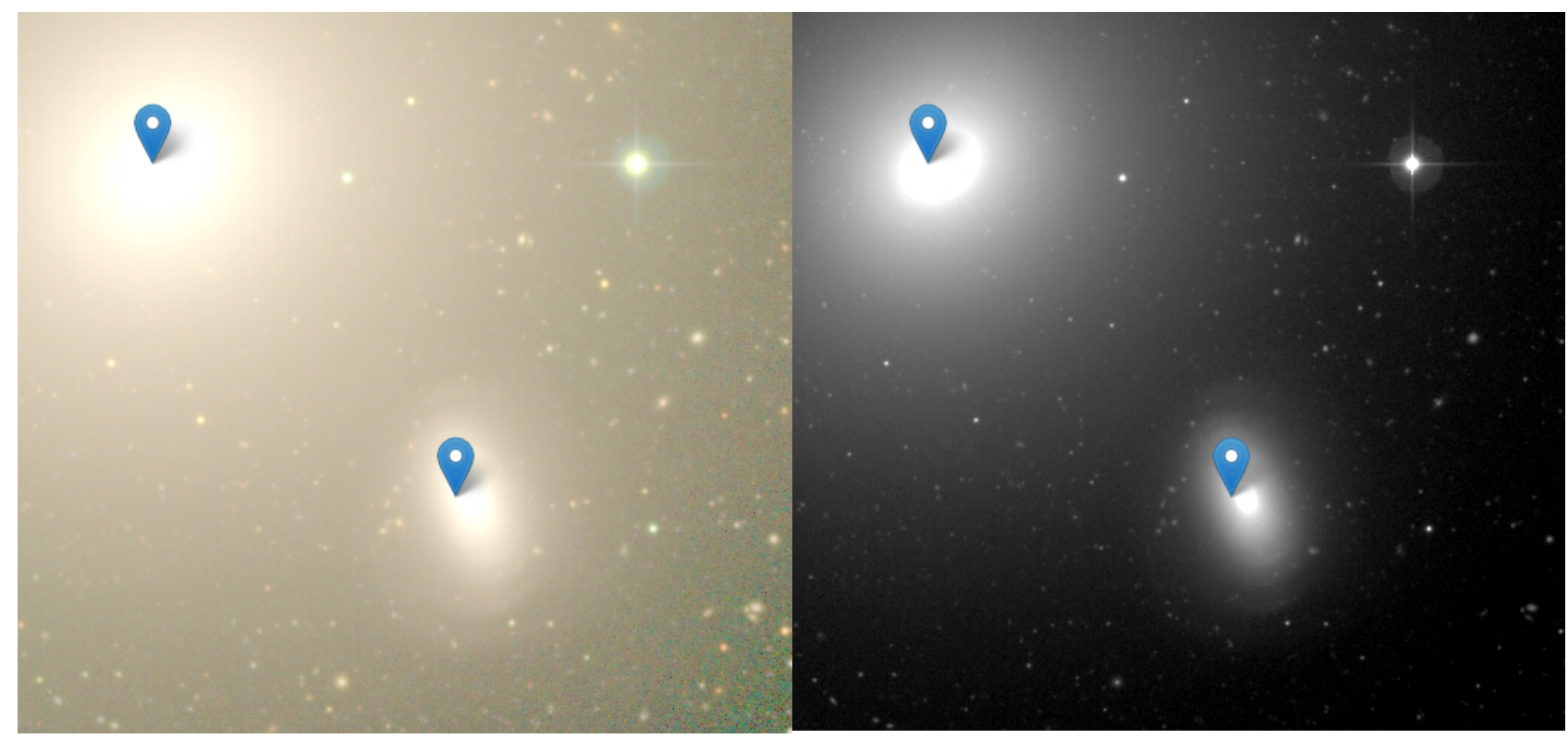

Figure B2. Shells of NGC 3605 (the smaller galaxy) are an example of a feature that was difficult to detect for the less experienced participants. Left panel shows the default view that was displayed to everyone. The right panel demonstrates that the shells are visible clearly after increasing the contrast of the image in the navigation tool.

Table B2. Census of the classified structures in the whole sample according to the rounded rating based on the votes of all participants (i.e., including also the less experienced ones) in percent.

\begin{tabular}{lllll}
\hline \hline Shells & no: $88 \pm 7$ & likely: $3 \pm 1$ & yes: $10 \pm 2$ & unknown: $0 \pm 0$ \\
Streams & no: $87 \pm 7$ & likely: $3 \pm 1$ & yes: $10 \pm 2$ & unknown: $0 \pm 0$ \\
Tails & no: $87 \pm 7$ & likely: $2 \pm 1$ & yes: $11 \pm 3$ & unknown: $0 \pm 0$ \\
Outer isophotes & regul.: $71 \pm 6$ & asym.: $11 \pm 2$ & disturb.: $18 \pm 3$ & unsure: $0 \pm 0$ \\
Tails or streams & no: $80 \pm 7$ & likely: $2 \pm 1$ & yes: $19 \pm 3$ & unknown: $0 \pm 0$ \\
Shells or streams or tails & no: $76 \pm 7$ & likely: $3 \pm 1$ & yes: $21 \pm 3$ & unknown: $0 \pm 0$ \\
Any tidal disturbance & no: $66 \pm 6$ & likely: $8 \pm 2$ & yes: $26 \pm 4$ & unknown: $0 \pm 0$ \\
Bars & no: $73 \pm 6$ & weak: $12 \pm 3$ & strong: $15 \pm 3$ & unsure: $0 \pm 0$ \\
Dust lanes & no: $86 \pm 7$ & weak: $6 \pm 2$ & strong: $8 \pm 2$ & unsure: $0 \pm 0$ \\
Peripheral disks & no: $96 \pm 7$ & & yes: $4 \pm 1$ & unsure: $0 \pm 0$ \\
Cirrus & no: $85 \pm 7$ & weak: $4 \pm 1$ & strong: $11 \pm 2$ & unsure: $0 \pm 0$ \\
\hline
\end{tabular}



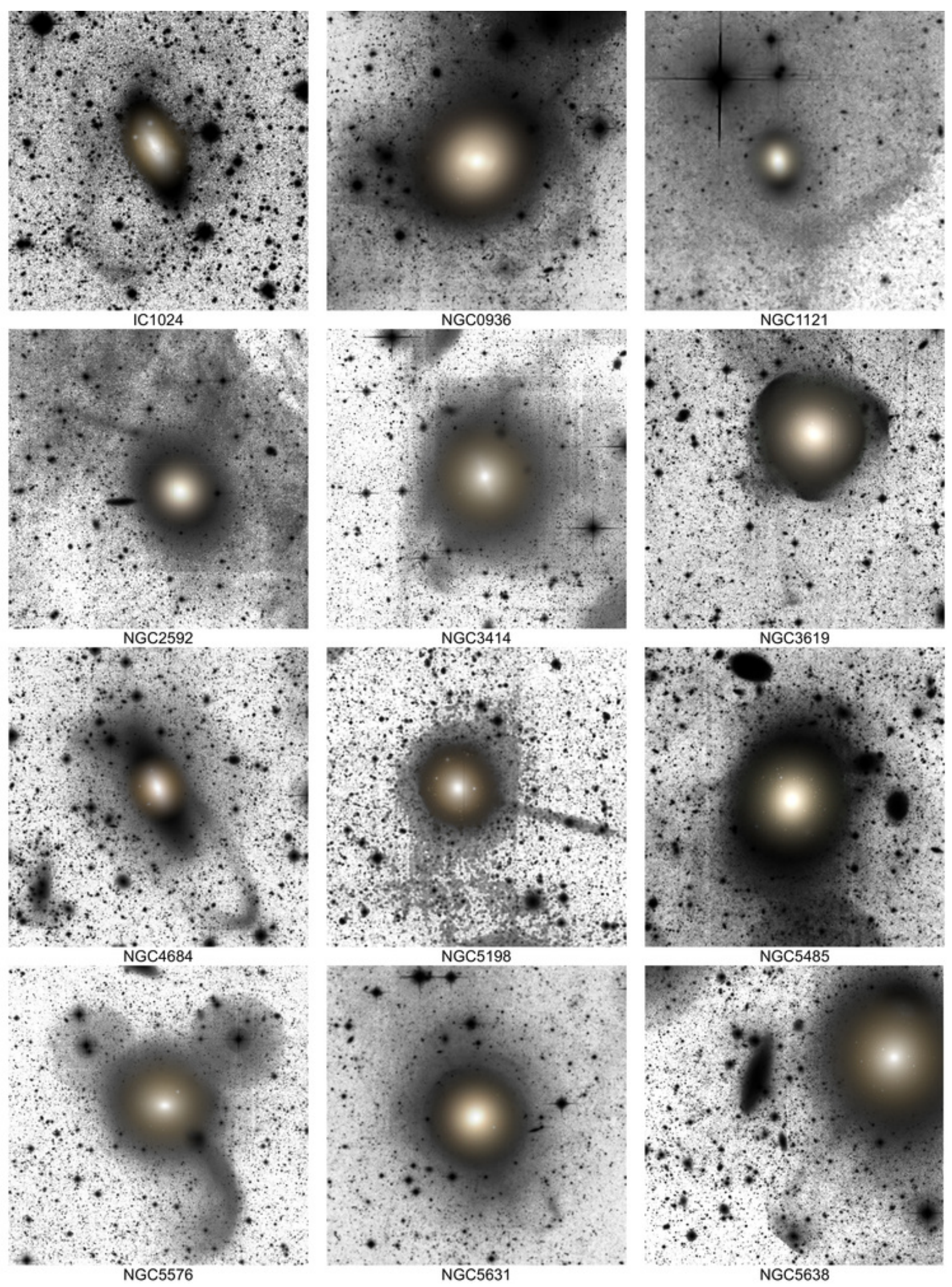

Figure C1. Examples of galaxies that were classified as having streams. 

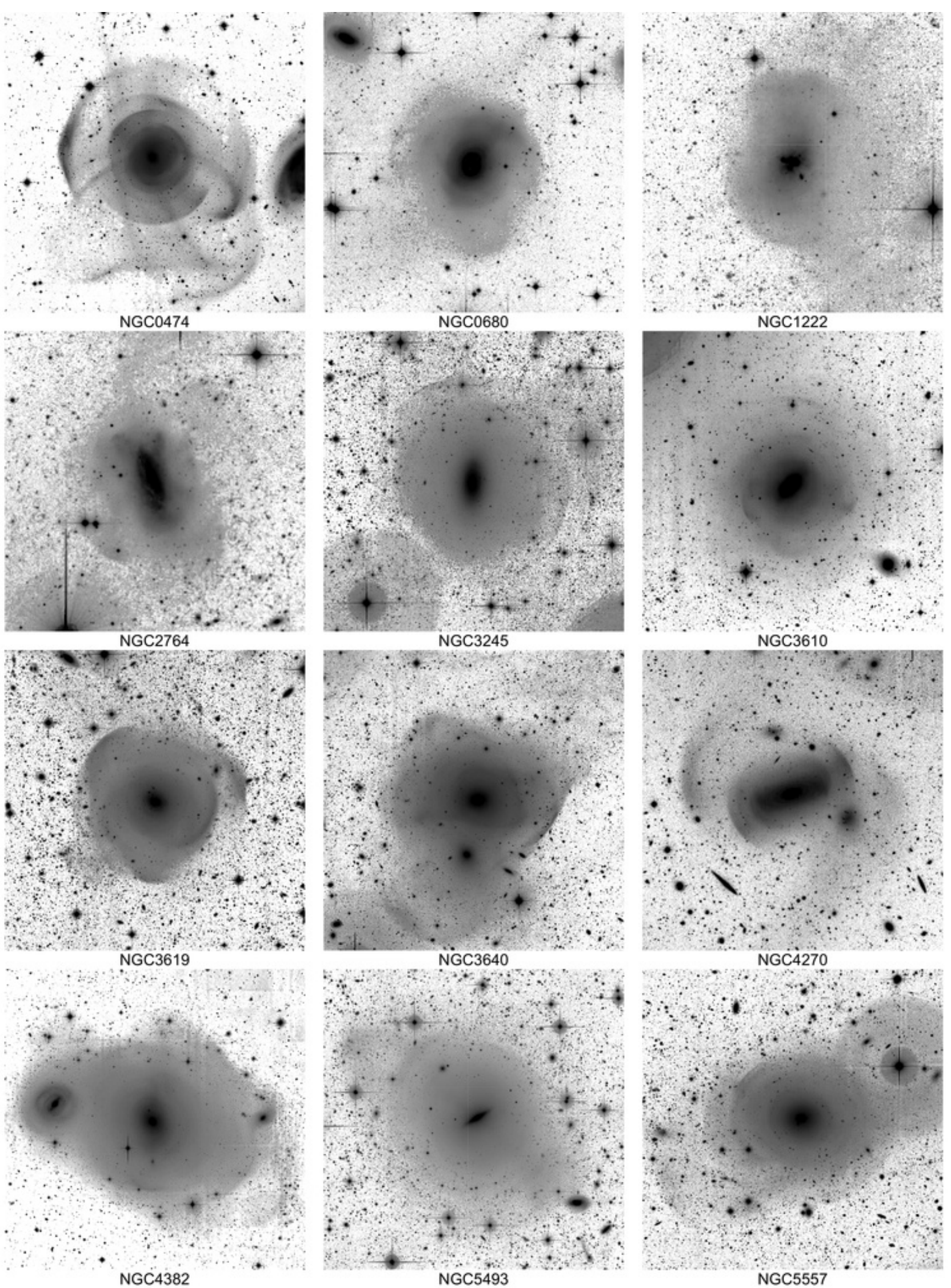

Figure C2. Examples of galaxies that were classified as having shells. 

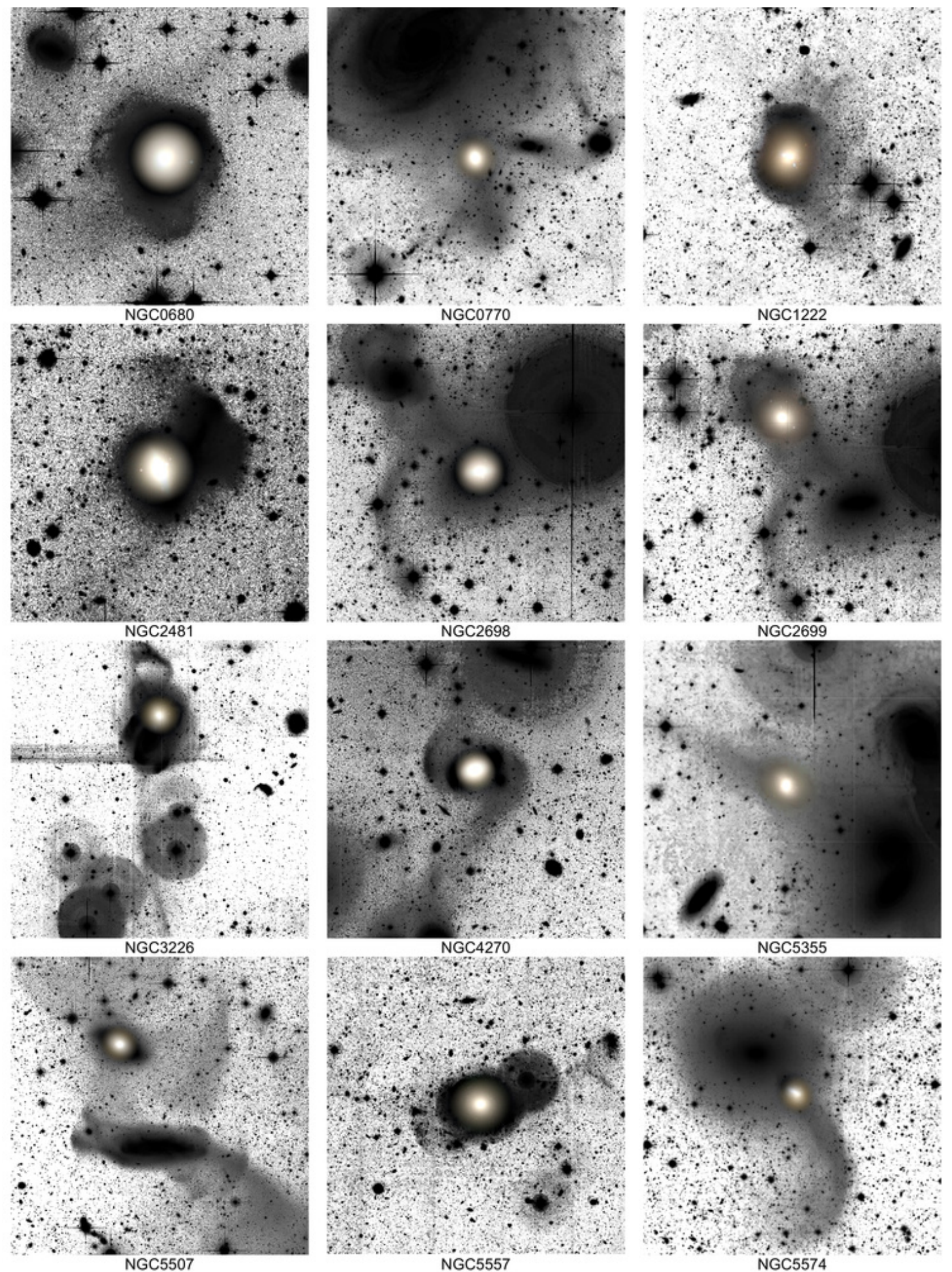

Figure C3. Examples of galaxies that were classified as having tidal tails. 


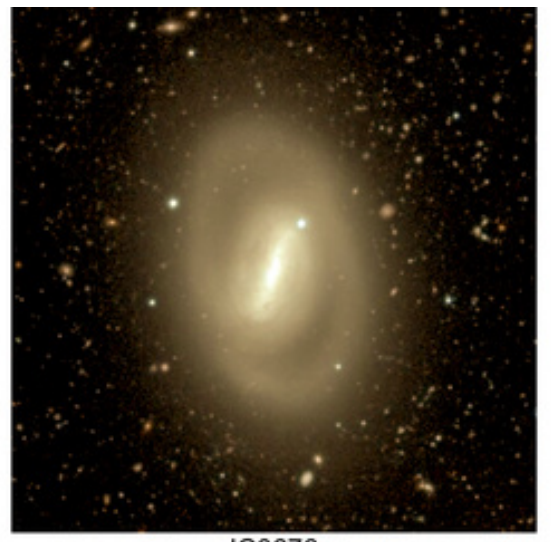

IC0676

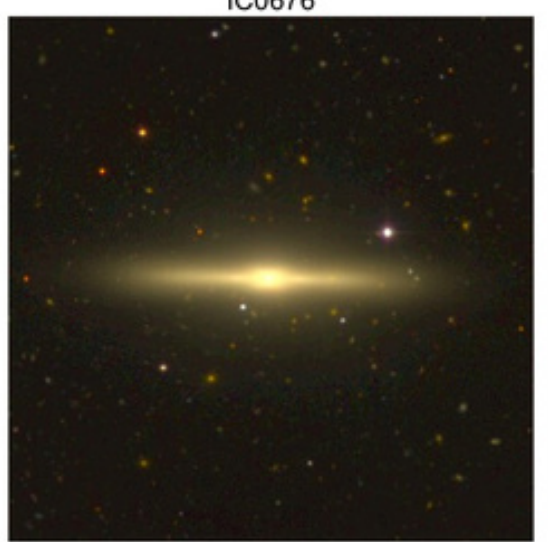

NGC3098

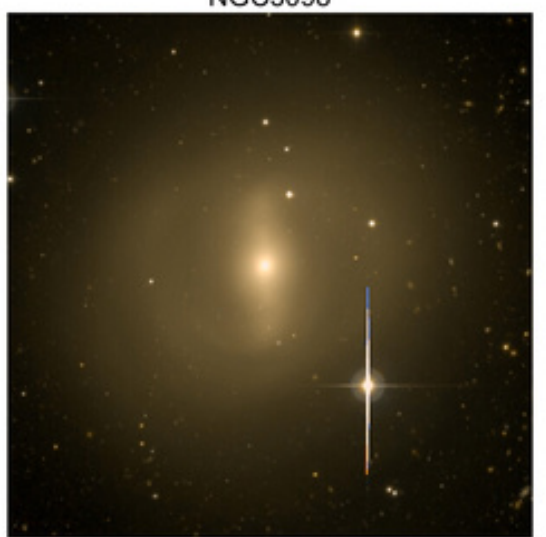

NGC4624

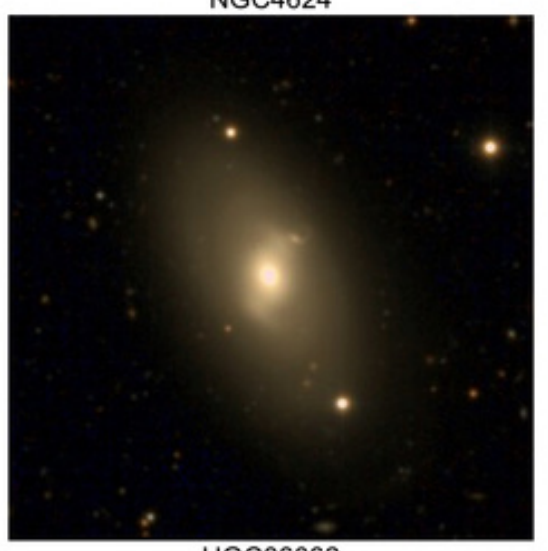

UGC06062

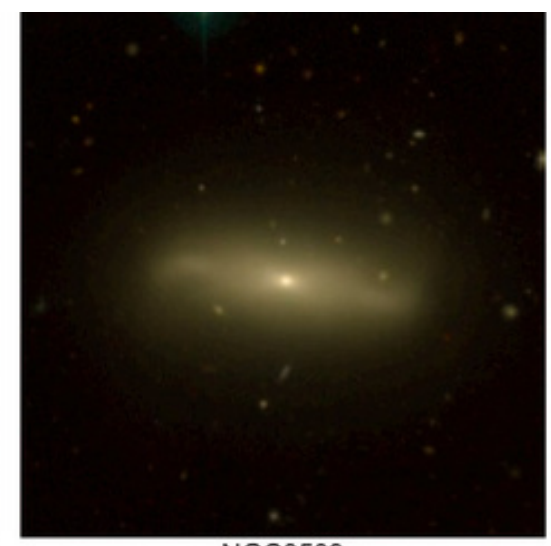

NGC0509

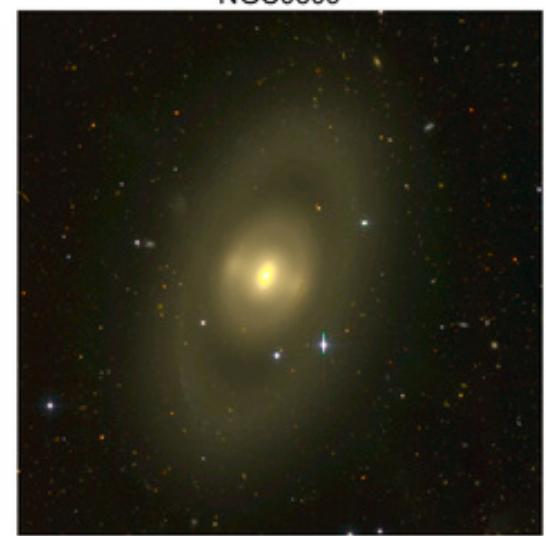

NGC3945

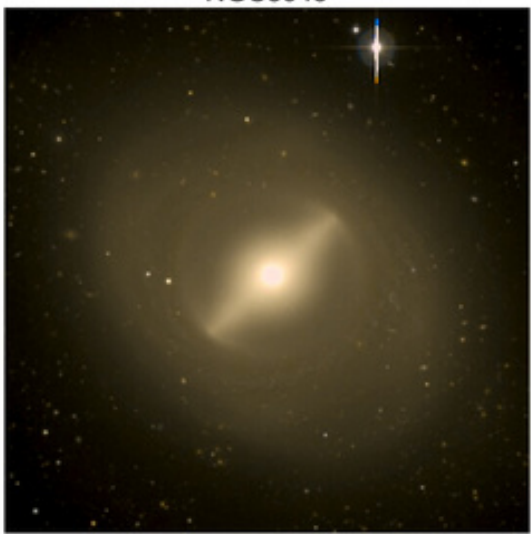

NGC4643

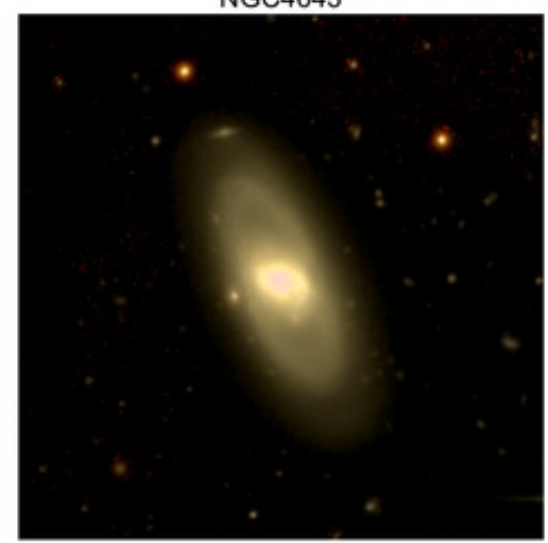

UGC06176

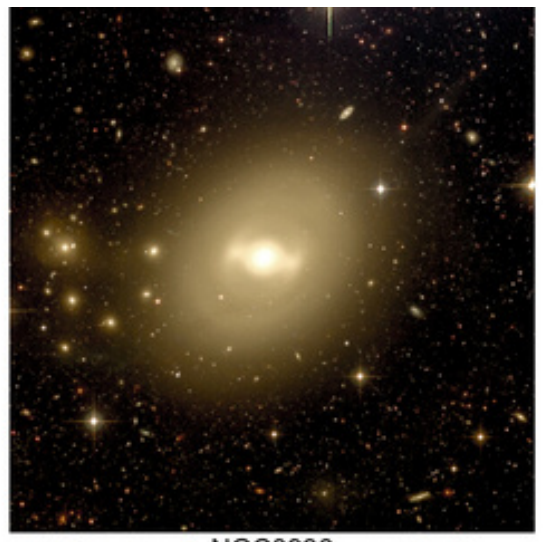

NGC0936

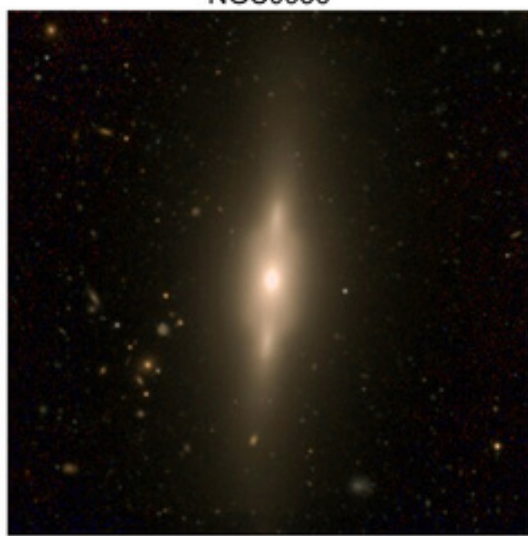

NGC4215

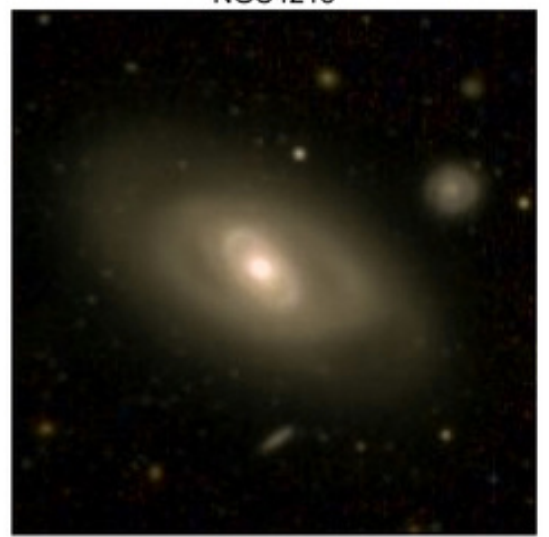

PGC042549

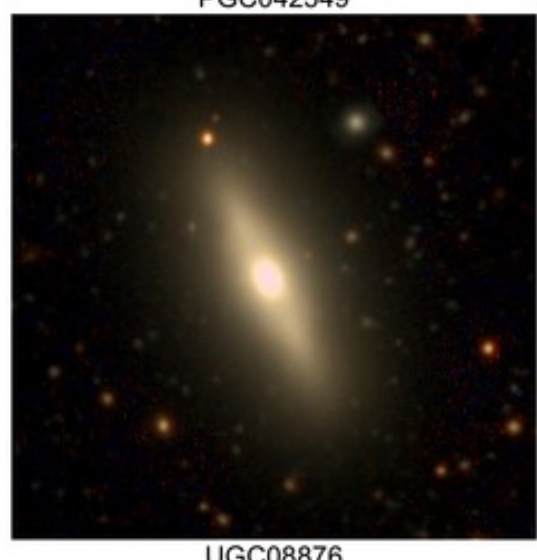

UGC08876

Figure C4. Examples of galaxies that were classified as having bars. 

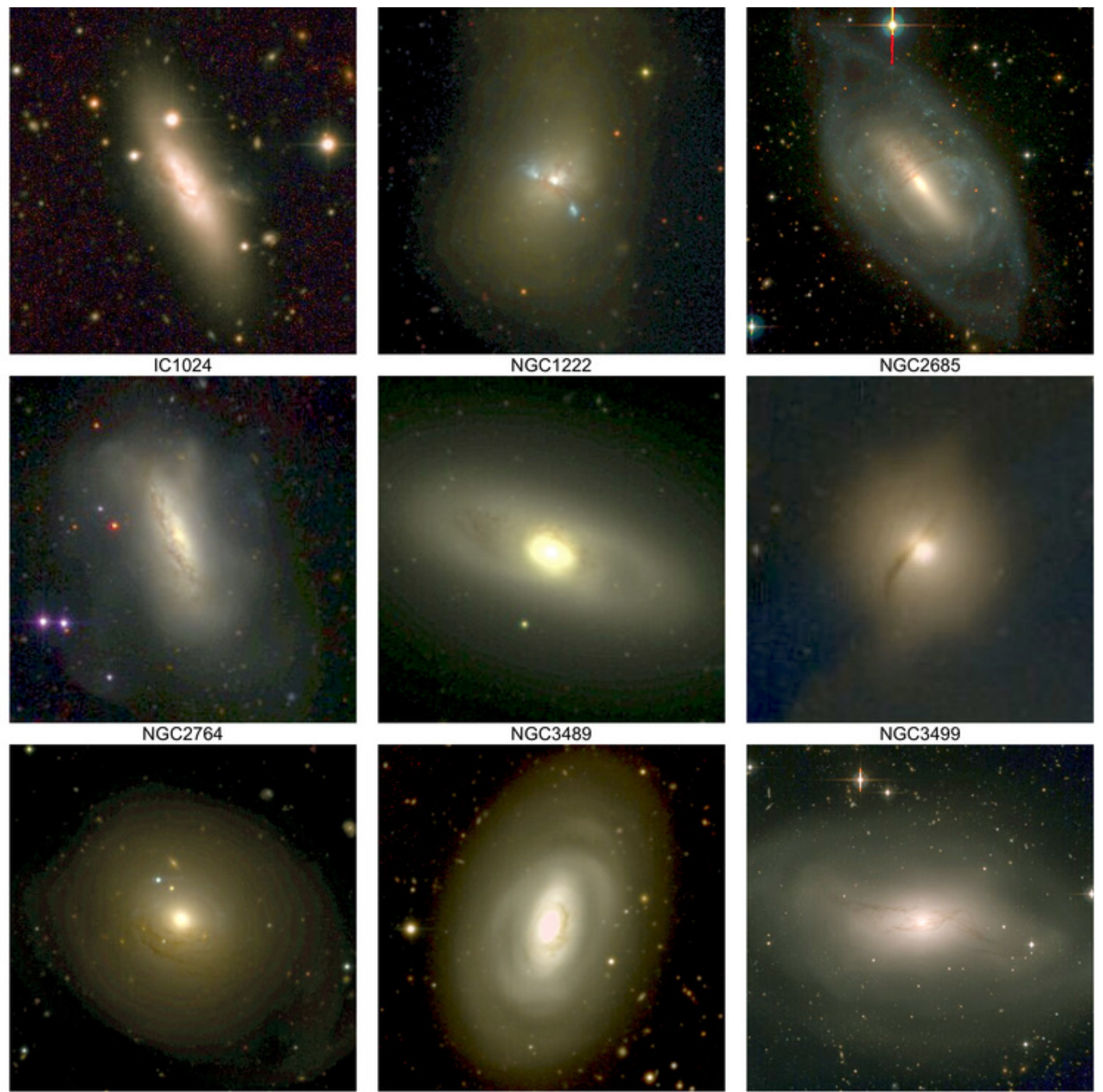

NGC3619
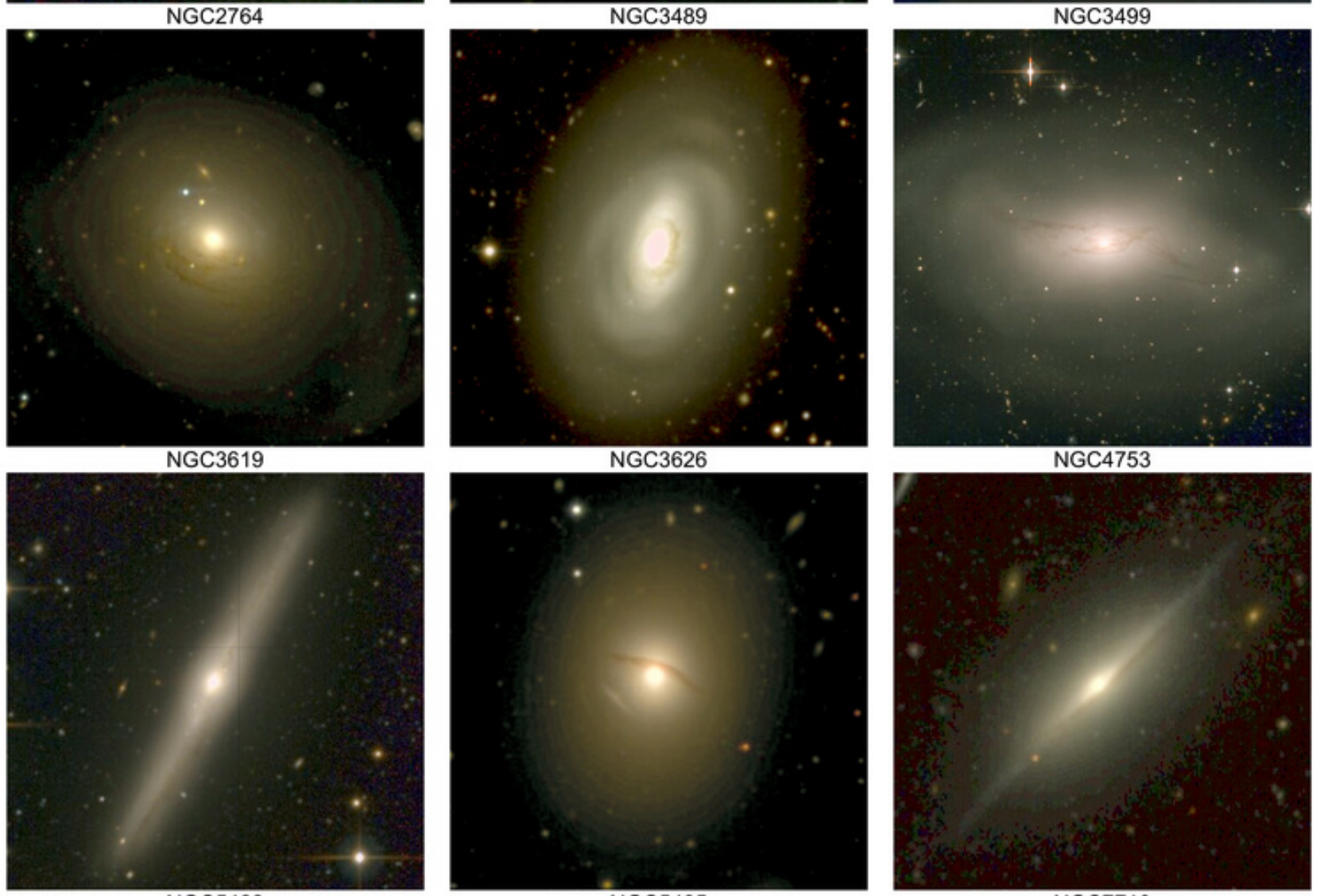

NGC5422
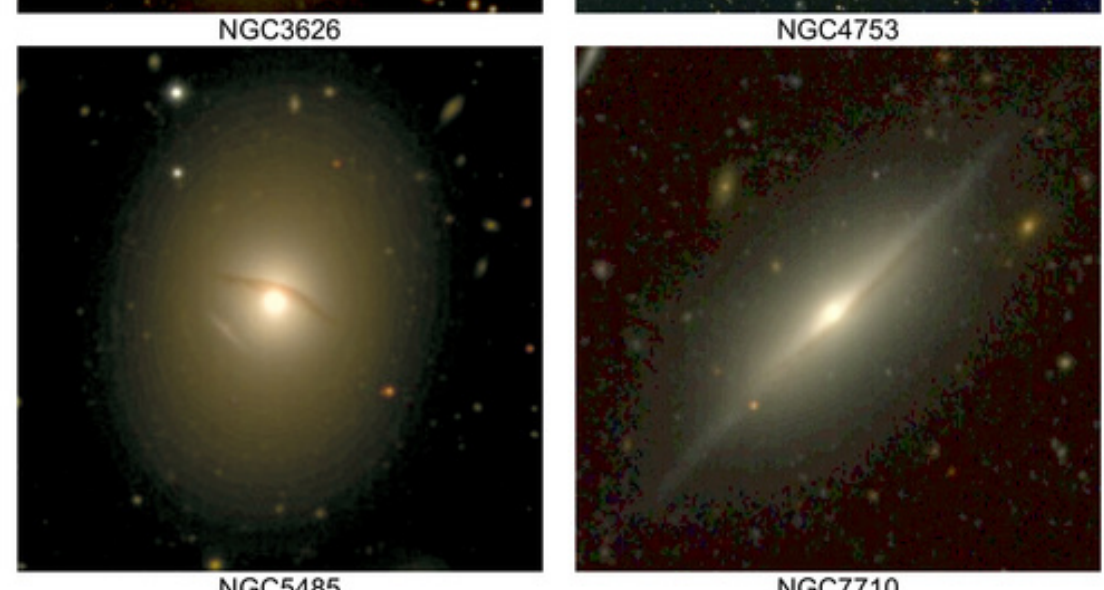

Figure C5. Examples of galaxies that were classified as having dust lanes. 


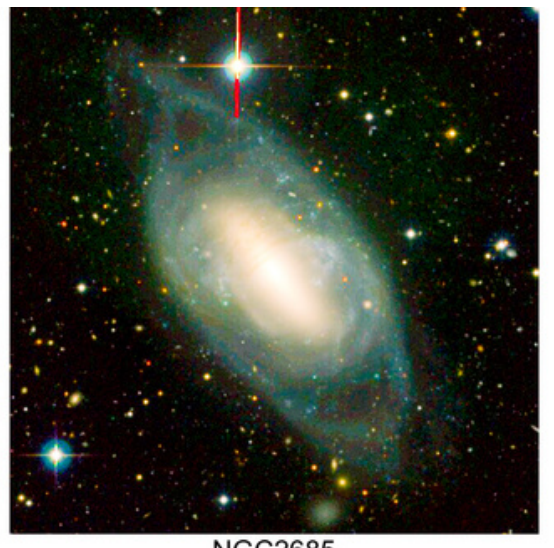

NGC2685

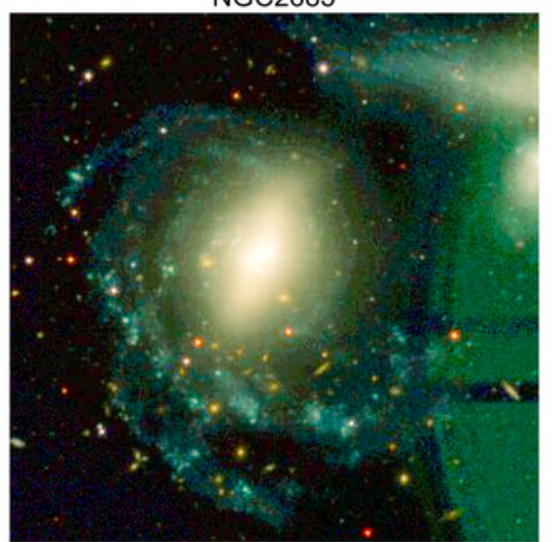

NGC7465

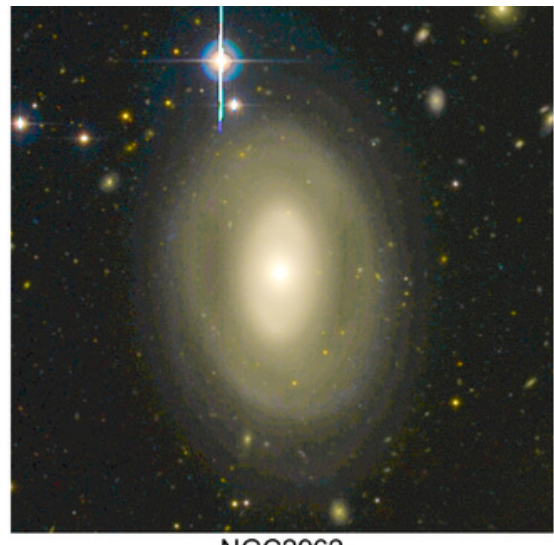

NGC2962

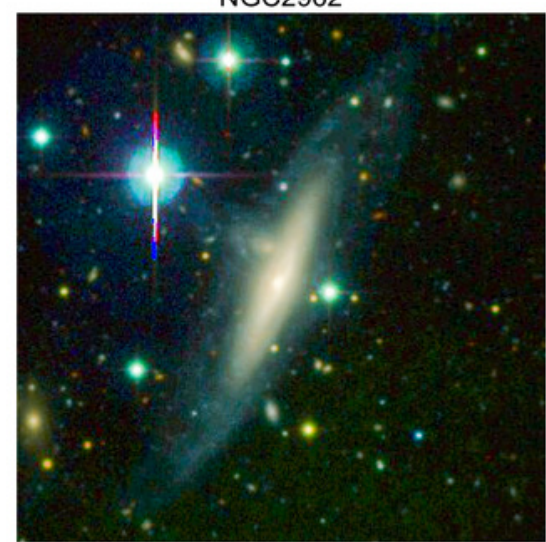

PGC016060

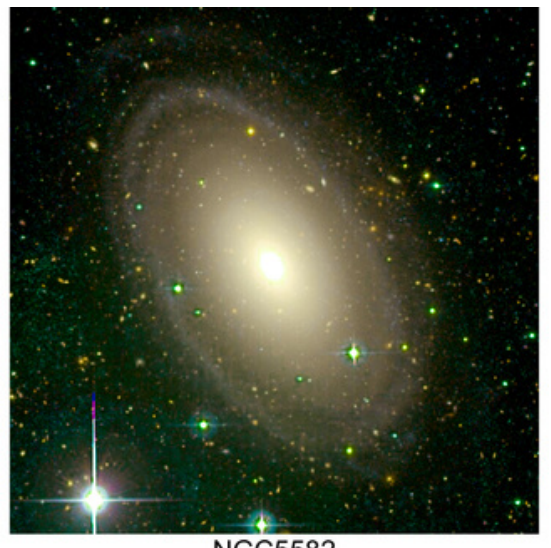

NGC5582

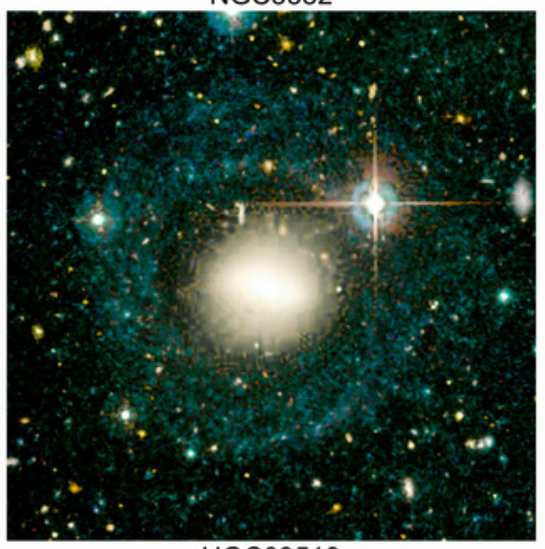

UGC09519

Figure C6. Examples of galaxies that were classified as having peripheral disks. 

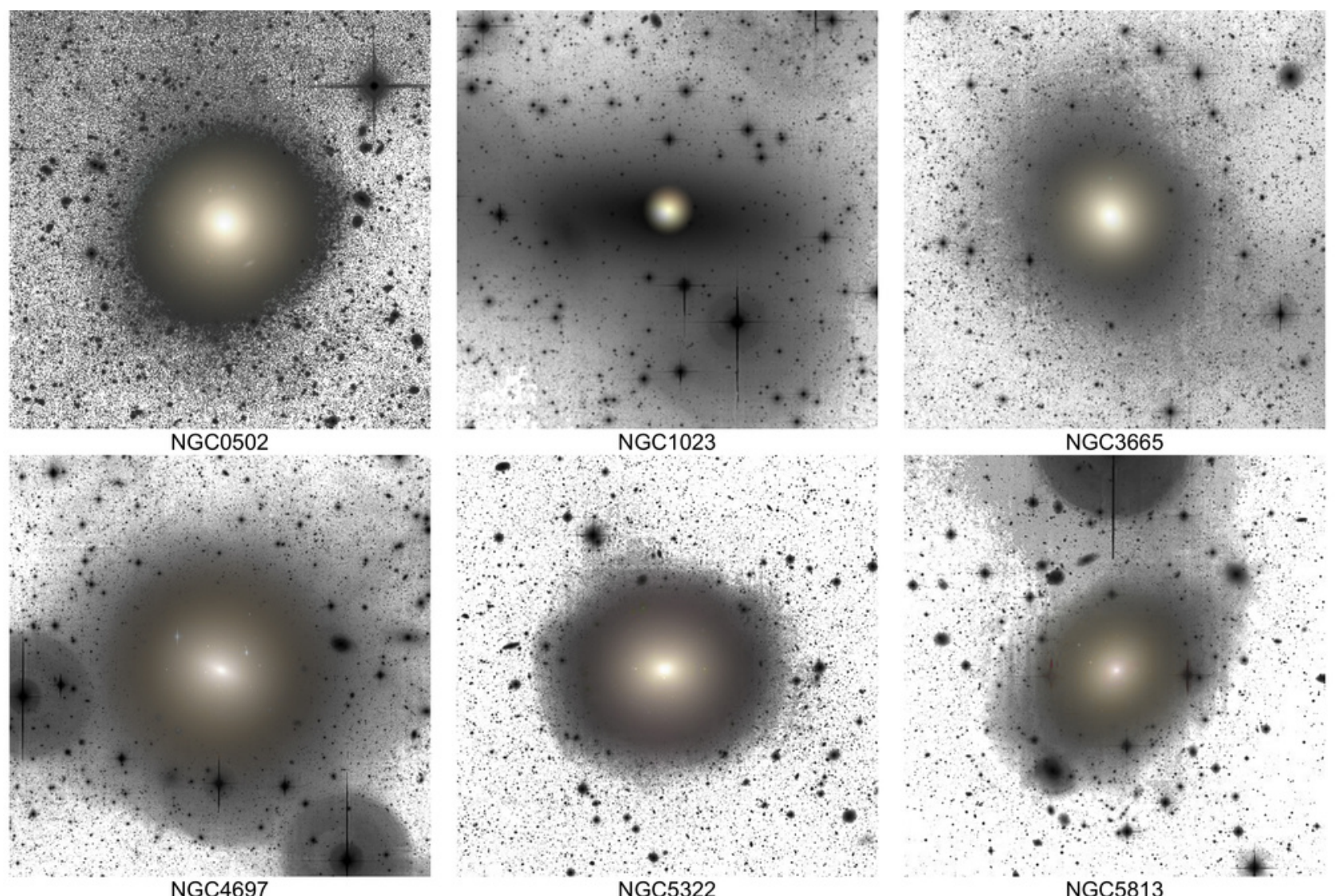

Figure C7. Examples of galaxies whose outer isophotes were rated as asymmetric, but that are devoid of prominent tidal features. The asymmetric classification signifies that the outer isophotes deviate from ellipses just mildly, according to the judgement of the participants. 


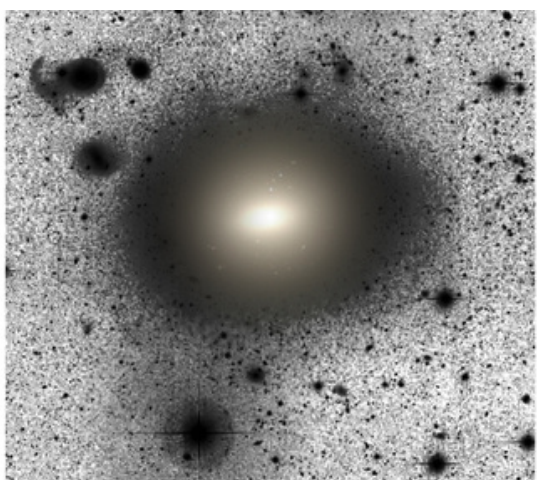

NGC3613

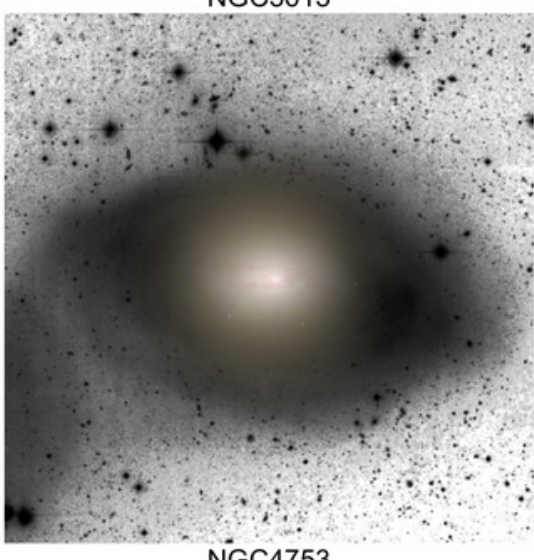

NGC4753

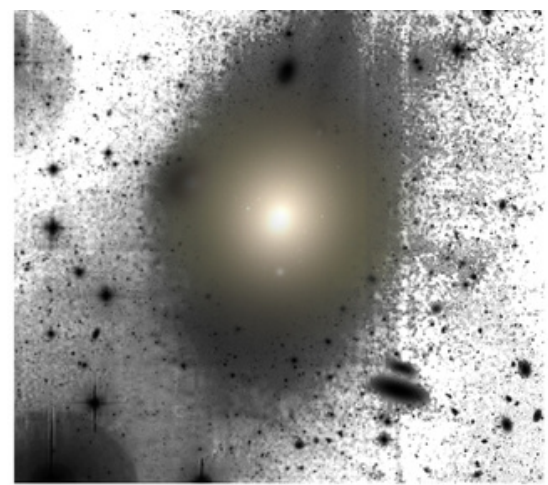

NGC4261

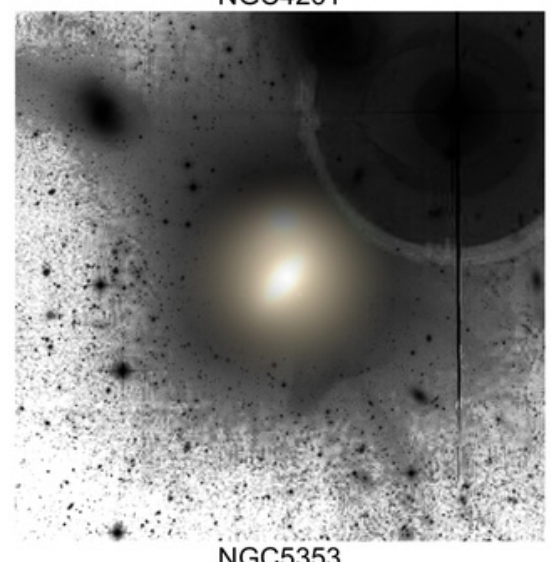

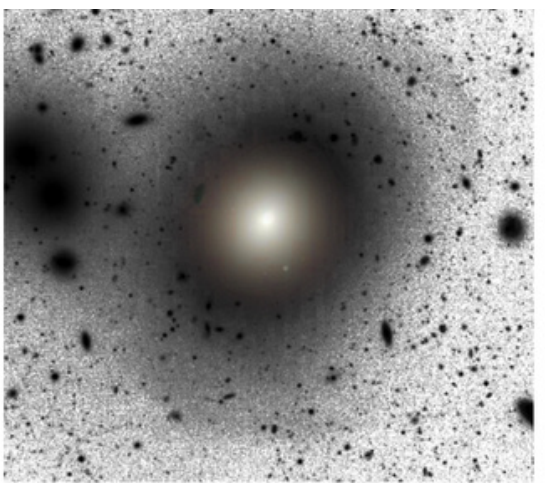

NGC4690

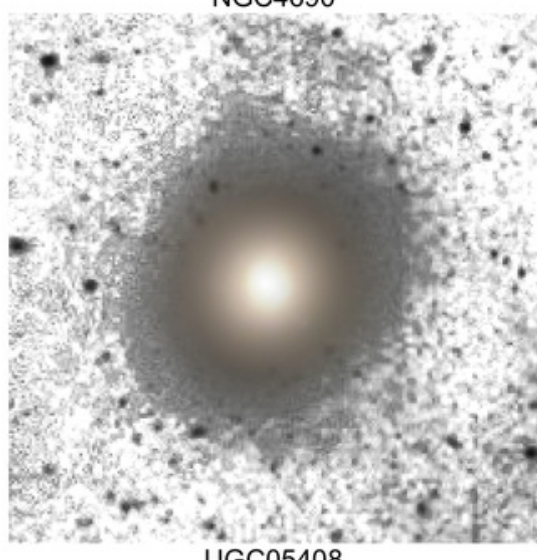

Figure C8. Examples of galaxies whose outer isophotes were rated as disturbed, but that are devoid of prominent tidal features. The disturbed classification signifies that the outer isophotes deviate from ellipses significantly, according to the judgement of the participants. 

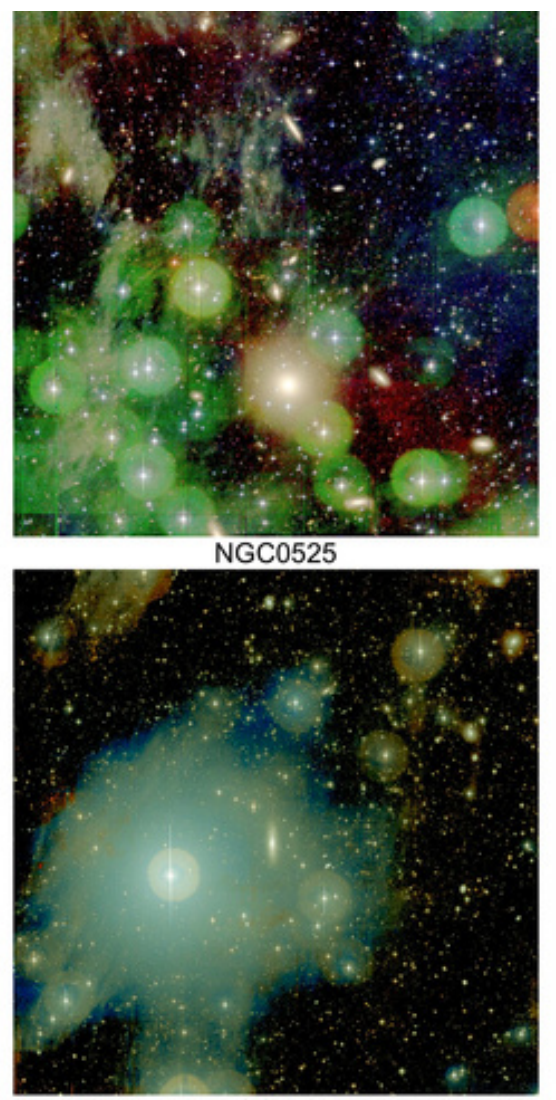

NGC2549

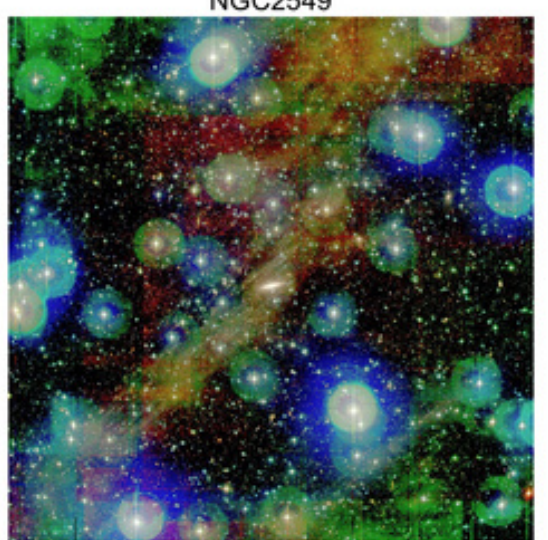

NGC6010

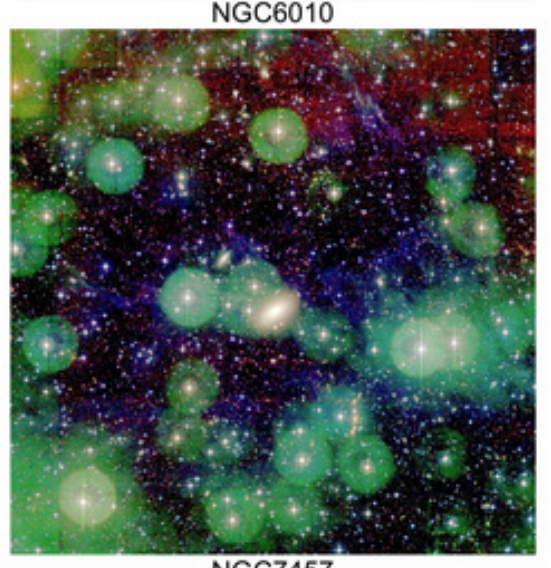

NGC7457
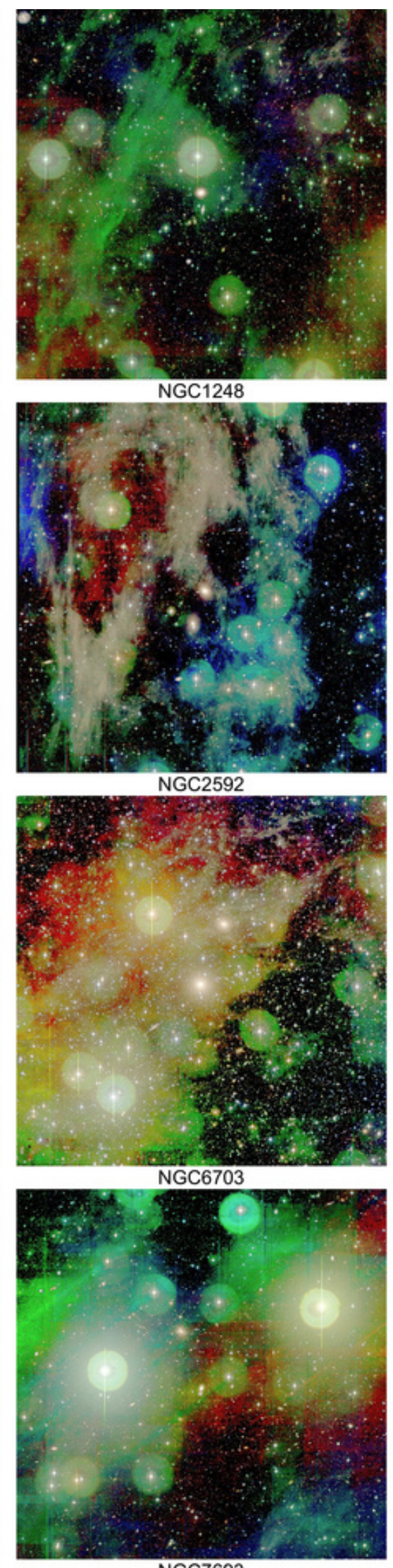

NGC7693

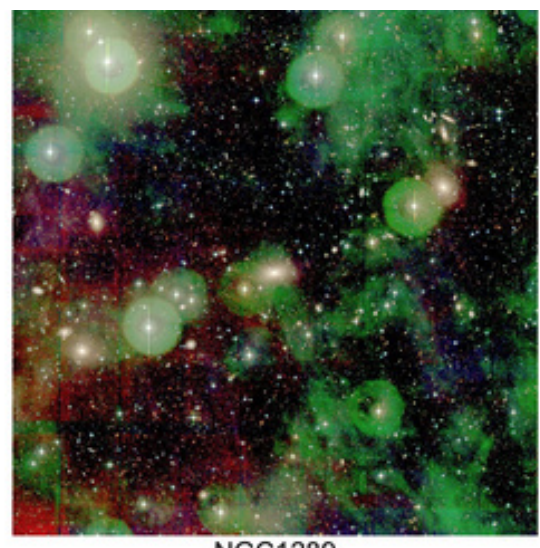

NGC1289

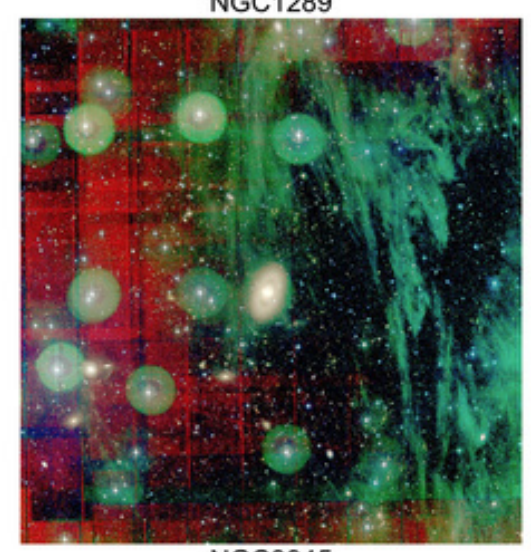

NGC3945

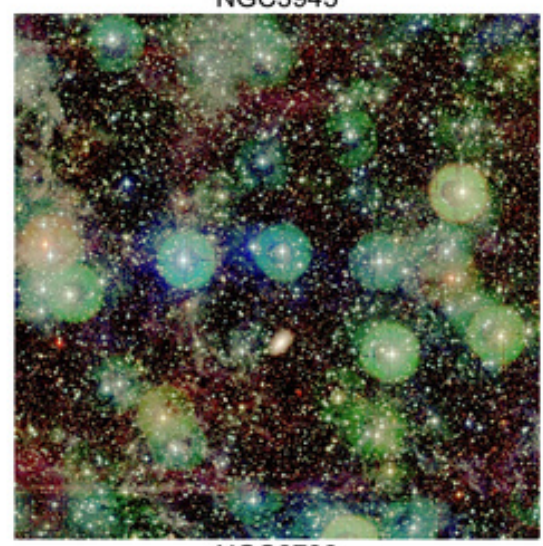

NGC6798

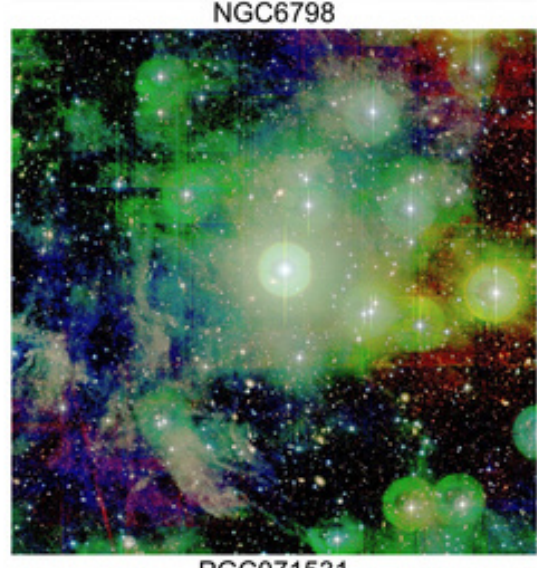

PGC071531

Figure C9. Examples of galaxies where the identification of the faint structure was complicated by the presence of strong Galactic cirri. 
APPENDIX D: PRESENCE OF MORPHOLOGICAL FEATURES IN INDIVIDUAL GALAXIES

Table D1: Ratings and classification codes for all galaxies.

\begin{tabular}{|c|c|c|c|c|c|c|c|c|c|c|}
\hline Name & "Classification code & Streams & "Shells & Tails & Ext. SF & Out. isoph. & Dust & Bars & "Halos & 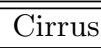 \\
\hline IC0560 & $+w b$ & 0.0 & 0.0 & 0.0 & 0.0 & 0.0 & 0.0 & 1.0 & 0.0 & 0.0 \\
\hline IC0598 & & 0.0 & 0.0 & 0.0 & 0.0 & 0.0 & 0.0 & 0.0 & 0.0 & 0.0 \\
\hline IC0676 & $+\mathrm{pb}$ & 0.0 & 0.0 & 0.0 & 0.0 & 0.0 & 0.0 & 2.0 & 0.0 & 0.0 \\
\hline IC1024 & $+\mathrm{s}+\mathrm{ph}+\mathrm{pl}$ & 2.0 & 0.0 & 0.0 & 0.0 & 2.0 & 2.0 & 0.0 & 0.0 & 0.0 \\
\hline NGC0448 & & 0.0 & 0.0 & 0.0 & 0.0 & 0.0 & 0.0 & 0.0 & 0.0 & 0.0 \\
\hline NGC0474 & $+\mathrm{s}+\mathrm{r}+\mathrm{t}+\mathrm{ph}-\mathrm{h}$ & 2.0 & 2.0 & 2.0 & 0.0 & 2.0 & 0.0 & 0.0 & 1.0 & 0.0 \\
\hline NGC0502 & $+\mathrm{r}+\mathrm{ah}$ & 0.0 & 2.0 & 0.0 & 0.0 & 1.0 & 0.0 & 0.0 & 0.0 & 0.0 \\
\hline NGC0509 & $+\mathrm{pb}$ & 0.0 & 0.0 & 0.0 & 0.0 & 0.0 & 0.0 & 2.0 & 0.0 & 0.0 \\
\hline NGC0516 & $+\mathrm{wb}$ & 0.0 & 0.0 & 0.0 & 0.0 & 0.0 & 0.0 & 1.0 & 0.0 & 0.0 \\
\hline NGC0524 & $+\mathrm{h} ?+\mathrm{wl}-\mathrm{pc}$ & 0.0 & 0.5 & 0.0 & 0.0 & -1 & 1.0 & 0.0 & 0.0 & 2.0 \\
\hline NGC0525 & $+w b-p c$ & 0.0 & 0.0 & 0.0 & 0.0 & 0.0 & 0.0 & 1.0 & 0.0 & 2.0 \\
\hline NGC0661 & & 0.0 & 0.0 & 0.0 & 0.0 & 0.0 & 0.0 & 0.0 & 0.0 & 0.0 \\
\hline NGC0680 & $+\mathrm{s}+\mathrm{r}+\mathrm{t}+\mathrm{ph}+\mathrm{wl}-\mathrm{h}-\mathrm{wc}$ & 2.0 & 2.0 & 2.0 & 0.0 & 2.0 & 1.0 & 0.0 & 1.0 & 1.0 \\
\hline NGC0770 & $+\mathrm{t}+\mathrm{ah}-\mathrm{h}-\mathrm{pc}$ & 0.0 & 0.0 & 2.0 & 0.0 & 1.0 & 0.0 & 0.0 & 1.0 & 2.0 \\
\hline NGC0821 & $-p c$ & 0.0 & 0.0 & 0.0 & 0.0 & 0.5 & 0.0 & 0.0 & 0.0 & 2.0 \\
\hline NGC0936 & $+\mathrm{s}+\mathrm{pb}$ & 2.0 & 0.0 & 0.0 & 0.0 & 0.0 & 0.0 & 2.0 & 0.0 & 0.0 \\
\hline NGC1023 & $+a h$ & 0.0 & 0.0 & 0.0 & 0.0 & 1.0 & 0.0 & 0.5 & 0.0 & 0.0 \\
\hline NGC1121 & $+\mathrm{s}$ & 2.0 & 0.0 & 0.0 & 0.0 & 0.0 & 0.0 & 0.0 & 0.0 & 0.0 \\
\hline NGC1222 & $+\mathrm{r}+\mathrm{t}+\mathrm{ph}+\mathrm{pl}$ & 0.0 & 2.0 & 2.0 & 0.0 & 2.0 & 2.0 & 0.0 & 0.0 & 0.0 \\
\hline NGC1248 & $-h-p c$ & 0.0 & 0.0 & 0.0 & 0.0 & 0.0 & 0.0 & 0.5 & 1.0 & 2.0 \\
\hline NGC1266 & +wl-wc & 0.0 & 0.0 & 0.0 & 0.0 & 0.0 & 1.0 & 0.0 & 0.0 & 1.0 \\
\hline NGC1289 & $-p c$ & 0.0 & 0.0 & 0.0 & 0.0 & 0.0 & 0.0 & 0.0 & 0.0 & 1.5 \\
\hline NGC1665 & $+w b-w c$ & 0.0 & 0.0 & 0.0 & 0.0 & 0.0 & 0.0 & 1.0 & 0.0 & 1.0 \\
\hline NGC2481 & $+\mathrm{t}+\mathrm{ph}-\mathrm{h}-\mathrm{pc}$ & 0.0 & 0.0 & 2.0 & 0.0 & 2.0 & 0.0 & 0.5 & 1.0 & 1.5 \\
\hline NGC2549 & $+\mathrm{wb}-\mathrm{pc}$ & 0.0 & 0.0 & 0.0 & 0.0 & 0.0 & 0.0 & 1.0 & 0.0 & 2.0 \\
\hline NGC2577 & $-\mathrm{wc}$ & 0.0 & 0.0 & 0.0 & 0.0 & 0.0 & 0.0 & 0.0 & 0.0 & 1.0 \\
\hline NGC2592 & $+\mathrm{s}-\mathrm{pc}$ & 2.0 & 0.0 & 0.0 & 0.0 & 0.0 & 0.0 & 0.0 & 0.0 & 2.0 \\
\hline NGC2594 & $+a h+w b-h-w c$ & 0.0 & 0.0 & 0.0 & 0.0 & 1.0 & 0.0 & 1.0 & 1.0 & 1.0 \\
\hline NGC2679 & $+w b$ & 0.0 & 0.0 & 0.0 & 0.0 & 0.0 & 0.0 & 1.0 & 0.0 & 0.0 \\
\hline NGC2685 & $+\mathrm{t} ?+\mathrm{d}+\mathrm{ah}+\mathrm{pl}$ & 0.0 & 0.0 & 1.0 & 1.0 & 1.0 & 2.0 & 0.5 & 0.0 & 0.0 \\
\hline NGC2695 & & 0.0 & 0.0 & 0.0 & 0.0 & 0.0 & 0.0 & 0.0 & 0.0 & 0.0 \\
\hline NGC2698 & $+\mathrm{t}+\mathrm{ah}-\mathrm{h}$ & 0.0 & 0.0 & 2.0 & 0.0 & 1.0 & 0.0 & 0.0 & 1.0 & 0.0 \\
\hline NGC2699 & $+\mathrm{r}+\mathrm{t}+\mathrm{ph}-\mathrm{h}$ & 0.0 & 2.0 & 2.0 & 0.0 & 2.0 & 0.0 & 0.0 & 1.0 & 0.5 \\
\hline NGC2764 & $+\mathrm{r}+\mathrm{ph}+\mathrm{pl}$ & 0.0 & 2.0 & 0.0 & 0.0 & 2.0 & 2.0 & 0.0 & 0.0 & 0.0 \\
\hline NGC2768 & $+\mathrm{s} ?+\mathrm{ah}+\mathrm{wb}$ & 1.0 & 0.0 & 0.0 & 0.0 & 1.0 & 0.0 & 1.0 & 0.0 & 0.0 \\
\hline NGC2778 & $-\mathrm{h}$ & 0.0 & 0.0 & 0.0 & 0.0 & 0.0 & 0.0 & 0.0 & 1.0 & 0.0 \\
\hline NGC2824 & $+\mathrm{d}$ & 0.0 & 0.0 & 0.0 & 0.5 & 0.0 & 0.0 & 0.0 & 0.0 & 0.0 \\
\hline NGC2852 & -h-wc & 0.0 & 0.0 & 0.0 & 0.0 & 0.0 & 0.0 & 0.0 & 1.0 & 1.0 \\
\hline NGC2859 & $+\mathrm{pb}$ & 0.0 & 0.0 & 0.0 & 0.0 & 0.0 & 0.0 & 1.5 & 0.0 & 0.0 \\
\hline NGC2880 & & 0.0 & 0.0 & 0.0 & 0.0 & 0.5 & 0.0 & 0.0 & 0.0 & 0.0 \\
\hline NGC2950 & $+w b$ & 0.0 & 0.0 & 0.0 & 0.0 & 0.0 & 0.0 & 1.0 & 0.0 & 0.0 \\
\hline NGC2962 & $+d+w b$ & 0.0 & 0.0 & 0.0 & 1.0 & 0.0 & 0.0 & 1.0 & 0.0 & 0.0 \\
\hline NGC2974 & & 0.0 & 0.0 & 0.0 & 0.0 & 0.5 & 0.0 & 0.0 & 0.0 & 0.0 \\
\hline NGC3032 & & 0.0 & 0.0 & 0.0 & 0.0 & 0.0 & 0.0 & 0.0 & 0.0 & 0.0 \\
\hline NGC3073 & $+\mathrm{ph}$ & 0.0 & 0.0 & 0.0 & 0.0 & 1.5 & 0.0 & 0.0 & 0.0 & 0.0 \\
\hline NGC3098 & $+\mathrm{pb}$ & 0.0 & 0.0 & 0.0 & 0.0 & 0.0 & 0.0 & 2.0 & 0.0 & 0.0 \\
\hline NGC3156 & $+\mathrm{wl}$ & 0.0 & 0.0 & 0.0 & 0.0 & 0.0 & 1.0 & 0.0 & 0.0 & 0.0 \\
\hline NGC3182 & & 0.0 & 0.0 & 0.0 & 0.0 & 0.0 & 0.0 & 0.0 & 0.0 & 0.0 \\
\hline NGC3193 & $-\mathrm{h}$ & 0.0 & 0.0 & 0.0 & 0.0 & 0.0 & 0.0 & 0.0 & 1.0 & 0.0 \\
\hline NGC3226 & $+\mathrm{s} ?+\mathrm{t}+\mathrm{ph}+\mathrm{wl}-\mathrm{h}$ & 1.0 & 0.5 & 2.0 & 0.0 & 2.0 & 1.0 & 0.0 & 1.0 & 0.0 \\
\hline NGC3230 & $+\mathrm{pb}-\mathrm{wc}$ & 0.0 & 0.0 & 0.0 & 0.0 & 0.0 & 0.0 & 2.0 & 0.0 & 1.0 \\
\hline NGC3245 & $+\mathrm{r}-\mathrm{h}$ & 0.0 & 2.0 & 0.0 & 0.0 & 0.0 & 0.0 & 0.0 & 1.0 & 0.0 \\
\hline NGC3248 & $+w b$ & 0.0 & 0.0 & 0.0 & 0.0 & 0.0 & 0.0 & 1.0 & 0.0 & 0.0 \\
\hline NGC3301 & & 0.0 & 0.0 & 0.0 & 0.0 & 0.0 & 0.0 & 0.5 & 0.0 & 0.0 \\
\hline NGC3377 & $-\mathrm{h}$ & 0.0 & 0.0 & 0.0 & 0.0 & 0.0 & 0.0 & 0.0 & 1.0 & 0.0 \\
\hline NGC3379 & $+\mathrm{r} ?-\mathrm{h}$ & 0.0 & 1.0 & 0.0 & 0.0 & 0.0 & 0.0 & 0.0 & 1.0 & 0.0 \\
\hline NGC3384 & + wb-h & 0.0 & 0.0 & 0.0 & 0.0 & 0.5 & 0.0 & 1.0 & 1.0 & 0.0 \\
\hline
\end{tabular}


Table D1: continued.

\begin{tabular}{|c|c|c|c|c|c|c|c|c|c|c|}
\hline Name & Classification code & Streams & Shells & Tails & Ext. SF & Out. isoph. & Dust & Bars & Halos & Cirrus \\
\hline NGC3400 & $+\mathrm{pb}$ & 0.0 & 0.0 & 0.0 & 0.0 & 0.0 & 0.0 & 2.0 & 0.0 & 0.0 \\
\hline NGC3412 & & 0.0 & 0.0 & 0.0 & 0.0 & 0.0 & 0.0 & 0.5 & 0.0 & 0.0 \\
\hline NGC3414 & $+\mathrm{s}+\mathrm{t} ?+\mathrm{ph}+\mathrm{wb}$ & 2.0 & 0.5 & 1.0 & 0.0 & 2.0 & 0.0 & 1.0 & 0.0 & 0.0 \\
\hline NGC3457 & $+\mathrm{wl}$ & 0.0 & 0.0 & 0.0 & 0.0 & 0.0 & 1.0 & 0.0 & 0.0 & 0.0 \\
\hline NGC3458 & & 0.0 & 0.0 & 0.0 & 0.0 & 0.0 & 0.0 & 0.5 & 0.0 & 0.0 \\
\hline NGC3489 & $+\mathrm{pl}+\mathrm{wb}$ & 0.0 & 0.0 & 0.0 & 0.0 & 0.0 & 1.5 & 1.0 & 0.0 & 0.0 \\
\hline NGC3499 & $+\mathrm{ah}+\mathrm{pl}$ & 0.0 & 0.0 & 0.0 & 0.0 & 1.0 & 2.0 & 0.0 & 0.0 & 0.0 \\
\hline NGC3522 & & 0.0 & 0.0 & 0.0 & 0.0 & 0.0 & 0.0 & 0.0 & 0.0 & 0.0 \\
\hline NGC3530 & & 0.0 & 0.0 & 0.0 & 0.0 & 0.0 & 0.0 & 0.0 & 0.0 & 0.0 \\
\hline NGC3599 & $+w b$ & 0.0 & 0.0 & 0.0 & 0.0 & 0.0 & 0.0 & 1.0 & 0.0 & 0.0 \\
\hline NGC3605 & $+\mathrm{r}+\mathrm{ph}-\mathrm{h}$ & 0.0 & 2.0 & 0.0 & 0.0 & 2.0 & 0.0 & 0.0 & 1.0 & 0.0 \\
\hline NGC3607 & $+\mathrm{ph}+\mathrm{wl}-\mathrm{h}$ & 0.0 & 0.0 & 0.5 & 0.0 & 2.0 & 1.0 & 0.0 & 1.0 & 0.0 \\
\hline NGC3608 & $+\mathrm{r} ?+\mathrm{ph}-\mathrm{h}$ & 0.0 & 1.0 & 0.0 & 0.0 & 1.5 & 0.0 & 0.0 & 1.0 & 0.0 \\
\hline NGC3610 & $+\mathrm{r}+\mathrm{ph}+\mathrm{wb}$ & 0.0 & 2.0 & 0.0 & 0.0 & 2.0 & 0.0 & 1.0 & 0.0 & 0.0 \\
\hline NGC3613 & $+\mathrm{s}+\mathrm{r} ?+\mathrm{ph}$ & 2.0 & 1.0 & 0.0 & 0.0 & 2.0 & 0.0 & 0.0 & 0.0 & 0.0 \\
\hline NGC3619 & $+\mathrm{s}+\mathrm{r}+\mathrm{t} ?+\mathrm{ph}+\mathrm{pl}$ & 2.0 & 2.0 & 1.0 & 0.0 & 2.0 & 2.0 & 0.0 & 0.0 & 0.0 \\
\hline NGC3626 & $+\mathrm{pl}+\mathrm{wb}$ & 0.0 & 0.0 & 0.0 & 0.0 & 0.0 & 2.0 & 1.0 & 0.0 & 0.0 \\
\hline NGC3630 & $+w b$ & 0.0 & 0.0 & 0.0 & 0.0 & 0.0 & 0.0 & 1.0 & 0.0 & 0.0 \\
\hline NGC3640 & $+\mathrm{s}+\mathrm{r}+\mathrm{ph}-\mathrm{h}-\mathrm{wc}$ & 2.0 & 2.0 & 0.0 & 0.0 & 2.0 & 0.0 & 0.0 & 1.0 & 1.0 \\
\hline NGC3641 & + ph-h-wc & 0.0 & 0.0 & 0.0 & 0.0 & 2.0 & 0.0 & 0.0 & 1.0 & 1.0 \\
\hline NGC3648 & & 0.0 & 0.0 & 0.0 & 0.0 & 0.0 & 0.0 & 0.0 & 0.0 & 0.0 \\
\hline NGC3658 & & 0.0 & 0.0 & 0.0 & 0.0 & 0.0 & 0.0 & 0.0 & 0.0 & 0.0 \\
\hline NGC3665 & $+a h$ & 0.0 & 0.0 & 0.0 & 0.0 & 1.0 & 0.5 & 0.0 & 0.0 & 0.5 \\
\hline NGC3674 & & 0.0 & 0.0 & 0.0 & 0.0 & 0.0 & 0.0 & 0.0 & 0.0 & 0.0 \\
\hline NGC3694 & & 0.0 & 0.0 & 0.0 & 0.0 & 0.0 & 0.0 & 0.0 & 0.0 & 0.0 \\
\hline NGC3757 & $+\mathrm{wb}$ & 0.0 & 0.0 & 0.0 & 0.0 & 0.0 & 0.0 & 1.0 & 0.0 & 0.0 \\
\hline NGC3796 & & 0.0 & 0.0 & 0.0 & 0.0 & 0.0 & 0.0 & 0.5 & 0.0 & 0.0 \\
\hline NGC3838 & & 0.0 & 0.0 & 0.0 & 0.0 & 0.0 & 0.0 & 0.0 & 0.0 & 0.0 \\
\hline NGC3941 & $+w b$ & 0.0 & 0.0 & 0.0 & 0.0 & 0.0 & 0.0 & 1.0 & 0.0 & 0.0 \\
\hline NGC3945 & $+\mathrm{pb}-\mathrm{pc}$ & 0.0 & 0.0 & 0.0 & 0.0 & 0.0 & 0.0 & 2.0 & 0.0 & 2.0 \\
\hline NGC3998 & $+a h-h$ & 0.0 & 0.5 & 0.0 & 0.0 & 1.0 & 0.0 & 0.0 & 1.0 & 0.0 \\
\hline NGC4026 & $+w b$ & 0.0 & 0.0 & 0.0 & 0.0 & 0.0 & 0.0 & 1.0 & 0.0 & 0.0 \\
\hline NGC4036 & $+\mathrm{s}+\mathrm{wl}+\mathrm{wb}$ & 2.0 & 0.0 & 0.0 & 0.0 & 0.0 & 1.0 & 1.0 & 0.0 & 0.0 \\
\hline NGC4078 & & 0.0 & 0.0 & 0.0 & 0.0 & 0.0 & 0.0 & 0.0 & 0.0 & 0.0 \\
\hline NGC4111 & $+\mathrm{t}-\mathrm{h}$ & 0.0 & 0.0 & 2.0 & 0.0 & 0.0 & 0.0 & 0.0 & 1.0 & 0.0 \\
\hline NGC4119 & & 0.0 & 0.0 & 0.0 & 0.0 & 0.0 & 0.0 & 0.0 & 0.0 & 0.0 \\
\hline NGC4150 & & 0.0 & 0.0 & 0.0 & 0.0 & 0.0 & 0.5 & 0.0 & 0.0 & 0.0 \\
\hline NGC4191 & & 0.0 & 0.0 & 0.0 & 0.0 & 0.0 & 0.0 & 0.0 & 0.0 & 0.0 \\
\hline NGC4203 & $+\mathrm{s} ?-\mathrm{h}$ & 1.0 & 0.0 & 0.0 & 0.0 & 0.0 & 0.0 & 0.5 & 1.0 & 0.0 \\
\hline NGC4215 & $+\mathrm{pb}$ & 0.0 & 0.0 & 0.0 & 0.0 & 0.0 & 0.0 & 2.0 & 0.0 & 0.0 \\
\hline NGC4249 & & 0.0 & 0.0 & 0.0 & 0.0 & 0.0 & 0.0 & 0.0 & 0.0 & 0.0 \\
\hline NGC4259 & $+\mathrm{wb}$ & 0.0 & 0.0 & 0.0 & 0.0 & 0.0 & 0.0 & 1.0 & 0.0 & 0.0 \\
\hline NGC4261 & $+\mathrm{r}+\mathrm{ph}-\mathrm{h}$ & 0.0 & 2.0 & 0.0 & 0.0 & 2.0 & 0.0 & 0.0 & 1.0 & 0.0 \\
\hline NGC4264 & $+\mathrm{pb}-\mathrm{h}$ & 0.0 & 0.0 & 0.0 & 0.0 & 0.5 & 0.0 & 1.5 & 1.0 & 0.0 \\
\hline NGC4268 & $+\mathrm{pb}$ & 0.0 & 0.0 & 0.0 & 0.0 & 0.5 & 0.0 & 2.0 & 0.0 & 0.0 \\
\hline NGC4270 & $+\mathrm{r}+\mathrm{t}+\mathrm{ph}-\mathrm{h}$ & 0.0 & 2.0 & 2.0 & 0.0 & 2.0 & 0.0 & 0.0 & 1.0 & 0.0 \\
\hline NGC4278 & $-\mathrm{h}$ & 0.0 & 0.0 & 0.0 & 0.0 & 0.0 & 0.0 & 0.0 & 1.0 & 0.0 \\
\hline NGC4281 & $-\mathrm{h}$ & 0.0 & 0.0 & 0.0 & 0.0 & 0.0 & 0.0 & 0.0 & 1.0 & 0.0 \\
\hline NGC4283 & $+\mathrm{r} ?+\mathrm{t} ?-\mathrm{h}$ & 0.0 & 1.0 & 1.0 & 0.0 & 0.0 & 0.0 & 0.0 & 1.0 & 0.0 \\
\hline NGC4382 & $+\mathrm{s}+\mathrm{r}+\mathrm{ph}-\mathrm{h}-\mathrm{wc}$ & 2.0 & 2.0 & 0.0 & 0.0 & 2.0 & 0.0 & 0.0 & 1.0 & 1.0 \\
\hline NGC4623 & $+\mathrm{wb}$ & 0.0 & 0.0 & 0.0 & 0.0 & 0.0 & 0.0 & 1.0 & 0.0 & 0.0 \\
\hline NGC4624 & $+\mathrm{ah}+\mathrm{pb}$ & 0.0 & 0.0 & 0.0 & 0.0 & 1.0 & 0.0 & 2.0 & 0.0 & 0.0 \\
\hline NGC4636 & $+a h$ & 0.0 & 0.0 & 0.0 & 0.0 & 1.0 & 0.0 & 0.0 & 0.0 & 0.0 \\
\hline NGC4643 & $+\mathrm{s} ?+\mathrm{t}+\mathrm{ph}+\mathrm{pb}$ & 1.0 & 0.0 & 1.5 & 0.0 & 2.0 & 0.0 & 2.0 & 0.0 & 0.0 \\
\hline NGC4684 & $+\mathrm{s}+\mathrm{ph}$ & 2.0 & 0.0 & 0.0 & 0.0 & 1.5 & 0.0 & 0.0 & 0.0 & 0.0 \\
\hline NGC4690 & $+\mathrm{r} ?+\mathrm{ph}-\mathrm{h}$ & 0.0 & 1.0 & 0.0 & 0.0 & 2.0 & 0.0 & 0.0 & 1.0 & 0.0 \\
\hline NGC4697 & $+a h$ & 0.0 & 0.0 & 0.0 & 0.0 & 1.0 & 0.0 & 0.0 & 0.0 & 0.0 \\
\hline NGC4753 & $+\mathrm{ph}+\mathrm{pl}$ & 0.0 & 0.0 & 0.0 & 0.0 & 2.0 & 2.0 & 0.0 & 0.0 & 0.0 \\
\hline NGC5173 & $+\mathrm{s} ?+\mathrm{r} ?+\mathrm{ah}-\mathrm{h}$ & 1.0 & 1.0 & 0.0 & 0.0 & 1.0 & 0.0 & 0.0 & 1.0 & 0.0 \\
\hline
\end{tabular}


Table D1: continued.

\begin{tabular}{|c|c|c|c|c|c|c|c|c|c|c|}
\hline Name & "Classification code & Streams & "Shells & Tails & Ext. SF & Out. isoph. & Dust & Bars & Halos & 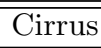 \\
\hline NGC5198 & $+\mathrm{s}+\mathrm{r}$ & 2.0 & 2.0 & 0.0 & 0.0 & 0.0 & 0.0 & 0.0 & 0.0 & 0.0 \\
\hline NGC5273 & & 0.0 & 0.5 & 0.0 & 0.0 & 0.0 & 0.0 & 0.0 & 0.0 & 0.0 \\
\hline NGC5308 & $+\mathrm{pb}$ & 0.0 & 0.0 & 0.0 & 0.0 & 0.5 & 0.0 & 2.0 & 0.0 & 0.0 \\
\hline NGC5322 & $+\mathrm{ah}$ & 0.0 & 0.0 & 0.0 & 0.0 & 1.0 & 0.0 & 0.0 & 0.0 & 0.0 \\
\hline NGC5342 & $+w b$ & 0.0 & 0.0 & 0.0 & 0.0 & 0.0 & 0.0 & 1.0 & 0.0 & 0.0 \\
\hline NGC5353 & $+\mathrm{t} ?+\mathrm{ph}-\mathrm{h}$ & 0.0 & 0.0 & 1.0 & 0.0 & 2.0 & 0.0 & 0.0 & 1.0 & 0.0 \\
\hline NGC5355 & $+\mathrm{s} ?+\mathrm{r} ?+\mathrm{t}+\mathrm{ph}-\mathrm{h}$ & 1.0 & 1.0 & 2.0 & 0.0 & 2.0 & 0.0 & 0.0 & 1.0 & 0.0 \\
\hline NGC5358 & $+\mathrm{pb}-\mathrm{h}$ & 0.0 & 0.0 & 0.0 & 0.0 & 0.0 & 0.0 & 1.5 & 1.0 & 0.0 \\
\hline NGC5379 & $+\mathrm{t}+\mathrm{ah}+\mathrm{wl}+\mathrm{pb}-\mathrm{h}$ & 0.0 & 0.0 & 2.0 & 0.0 & 1.0 & 1.0 & 2.0 & 1.0 & 0.0 \\
\hline NGC5422 & $+\mathrm{pl}+\mathrm{wb}$ & 0.0 & 0.0 & 0.0 & 0.0 & 0.0 & 2.0 & 1.0 & 0.0 & 0.0 \\
\hline NGC5473 & $+\mathrm{wb}$ & 0.0 & 0.0 & 0.0 & 0.0 & 0.0 & 0.0 & 1.0 & 0.0 & 0.0 \\
\hline NGC5481 & $-h-w c$ & 0.0 & 0.0 & 0.0 & 0.0 & 0.0 & 0.0 & 0.0 & 1.0 & 1.0 \\
\hline NGC5485 & $+\mathrm{s}+\mathrm{r} ?+\mathrm{ph}+\mathrm{pl}$ & 2.0 & 1.0 & 0.0 & 0.0 & 2.0 & 2.0 & 0.0 & 0.0 & 0.0 \\
\hline NGC5493 & $+\mathrm{r}+\mathrm{ph}$ & 0.0 & 2.0 & 0.0 & 0.0 & 2.0 & 0.0 & 0.0 & 0.0 & 0.0 \\
\hline NGC5500 & & 0.0 & 0.0 & 0.0 & 0.0 & 0.0 & 0.0 & 0.0 & 0.0 & 0.0 \\
\hline NGC5507 & $+\mathrm{r}+\mathrm{t}+\mathrm{ah}+\mathrm{wb}-\mathrm{h}$ & 0.0 & 2.0 & 2.0 & 0.0 & 1.0 & 0.0 & 1.0 & 1.0 & 0.0 \\
\hline NGC5557 & $+\mathrm{r}+\mathrm{t}+\mathrm{ph}$ & 0.0 & 2.0 & 2.0 & 0.0 & 2.0 & 0.0 & 0.0 & 0.0 & 0.0 \\
\hline NGC5574 & $+t+p h+w b-h$ & 0.0 & 0.0 & 2.0 & 0.0 & 2.0 & 0.0 & 1.0 & 1.0 & 0.0 \\
\hline NGC5576 & $+\mathrm{s}+\mathrm{t} ?+\mathrm{ph}-\mathrm{h}$ & 2.0 & 0.0 & 1.0 & 0.0 & 2.0 & 0.0 & 0.0 & 1.0 & 0.0 \\
\hline NGC5582 & $+\mathrm{d}$ & 0.0 & 0.0 & 0.0 & 1.0 & 0.0 & 0.0 & 0.0 & 0.0 & 0.0 \\
\hline NGC5611 & $+\mathrm{ah}+\mathrm{pb}$ & 0.0 & 0.0 & 0.0 & 0.0 & 1.0 & 0.0 & 2.0 & 0.0 & 0.0 \\
\hline NGC5631 & $+\mathrm{s}+\mathrm{r}+\mathrm{ph}+\mathrm{wl}$ & 2.0 & 2.0 & 0.0 & 0.0 & 2.0 & 1.0 & 0.0 & 0.0 & 0.0 \\
\hline NGC5638 & $+\mathrm{s}+\mathrm{r}-\mathrm{h}$ & 2.0 & 2.0 & 0.0 & 0.0 & 0.5 & 0.0 & 0.0 & 1.0 & 0.0 \\
\hline NGC5813 & $+a h$ & 0.0 & 0.0 & 0.0 & 0.0 & 1.0 & 0.0 & 0.0 & 0.0 & 0.0 \\
\hline NGC5831 & & 0.0 & 0.0 & 0.0 & 0.0 & 0.0 & 0.0 & 0.0 & 0.0 & 0.0 \\
\hline NGC5838 & $+\mathrm{pb}$ & 0.0 & 0.0 & 0.0 & 0.0 & 0.0 & 0.0 & 2.0 & 0.0 & 0.0 \\
\hline NGC5839 & $+w b$ & 0.0 & 0.0 & 0.0 & 0.0 & 0.0 & 0.0 & 1.0 & 0.0 & 0.0 \\
\hline NGC5845 & $+a h-h$ & 0.0 & 0.0 & 0.0 & 0.0 & 1.0 & 0.0 & 0.0 & 1.0 & 0.0 \\
\hline NGC5846 & $-\mathrm{h}$ & 0.0 & 0.0 & 0.0 & 0.0 & 0.5 & 0.0 & 0.0 & 1.0 & 0.0 \\
\hline NGC5866 & $+\mathrm{ph}+\mathrm{pb}$ & 0.0 & 0.0 & 0.0 & 0.0 & 2.0 & 0.0 & 2.0 & 0.0 & 0.0 \\
\hline NGC6010 & $+\mathrm{pb}-\mathrm{pc}$ & 0.0 & 0.0 & 0.0 & 0.0 & 0.0 & 0.0 & 2.0 & 0.0 & 2.0 \\
\hline NGC6014 & $+\mathrm{wl}+\mathrm{wb}$ & 0.0 & 0.0 & 0.0 & 0.0 & 0.0 & 1.0 & 1.0 & 0.0 & 0.5 \\
\hline NGC6017 & $+a h+w b$ & 0.0 & 0.0 & 0.0 & 0.0 & 1.0 & 0.0 & 1.0 & 0.0 & 0.0 \\
\hline NGC6278 & $+\mathrm{s} ?+\mathrm{wb}-\mathrm{h}$ & 1.0 & 0.0 & 0.0 & 0.0 & 0.0 & 0.0 & 1.0 & 1.0 & 0.0 \\
\hline NGC6547 & & 0.0 & 0.0 & 0.0 & 0.0 & 0.0 & 0.0 & 0.0 & 0.0 & 0.0 \\
\hline NGC6548 & $+\mathrm{pb}-\mathrm{pc}$ & 0.0 & 0.0 & 0.0 & 0.0 & 0.0 & 0.0 & 2.0 & 0.0 & 2.0 \\
\hline NGC6703 & $-p c$ & 0.0 & 0.0 & 0.0 & 0.0 & 0.0 & 0.0 & 0.0 & 0.0 & 2.0 \\
\hline NGC6798 & $+\mathrm{pb}-\mathrm{pc}$ & 0.0 & 0.0 & 0.0 & 0.0 & 0.0 & 0.0 & 2.0 & 0.0 & 2.0 \\
\hline NGC7280 & $+\mathrm{s} ?+\mathrm{pb}-\mathrm{h}$ & 1.0 & 0.0 & 0.0 & 0.0 & 0.0 & 0.0 & 2.0 & 1.0 & 0.0 \\
\hline NGC7332 & $+\mathrm{ah}+\mathrm{pb}-\mathrm{h}$ & 0.0 & 0.0 & 0.0 & 0.0 & 1.0 & 0.0 & 2.0 & 1.0 & 0.0 \\
\hline NGC7454 & $-p c$ & 0.0 & 0.0 & 0.0 & 0.0 & 0.0 & 0.0 & 0.0 & 0.0 & 2.0 \\
\hline NGC7457 & $-h-p c$ & 0.0 & 0.0 & 0.0 & 0.0 & 0.0 & 0.5 & 0.5 & 1.0 & 2.0 \\
\hline NGC7465 & $+\mathrm{t}+\mathrm{d}+\mathrm{ph}+\mathrm{pl}+\mathrm{pb}-\mathrm{h}-\mathrm{wc}$ & 0.0 & 0.0 & 2.0 & 1.0 & 2.0 & 2.0 & 2.0 & 1.0 & 1.0 \\
\hline NGC7693 & + wb-h-pc & 0.0 & 0.0 & 0.0 & 0.0 & 0.5 & 0.0 & 1.0 & 1.0 & 2.0 \\
\hline NGC7710 & $+\mathrm{pl}$ & 0.0 & 0.0 & 0.0 & 0.0 & 0.0 & 2.0 & 0.0 & 0.0 & 0.0 \\
\hline PGC016060 & $+\mathrm{d}+\mathrm{pl}-\mathrm{h}$ & 0.0 & 0.0 & 0.0 & 1.0 & 0.0 & 2.0 & 0.0 & 1.0 & 0.0 \\
\hline PGC028887 & $+\mathrm{s} ?$ & 1.0 & 0.0 & 0.0 & 0.0 & 0.0 & 0.0 & 0.0 & 0.0 & 0.0 \\
\hline PGC029321 & & 0.0 & 0.0 & 0.0 & 0.0 & 0.0 & 0.0 & 0.0 & 0.0 & 0.0 \\
\hline PGC042549 & $+\mathrm{pb}$ & 0.0 & 0.0 & 0.0 & 0.0 & 0.0 & 0.5 & 2.0 & 0.0 & 0.0 \\
\hline PGC050395 & & 0.0 & 0.0 & 0.0 & 0.0 & 0.0 & 0.0 & 0.0 & 0.0 & 0.0 \\
\hline PGC056772 & & 0.0 & 0.0 & 0.0 & 0.0 & 0.0 & 0.0 & 0.0 & 0.0 & 0.0 \\
\hline PGC058114 & $+\mathrm{wl}+\mathrm{wb}$ & 0.0 & 0.0 & 0.0 & 0.0 & 0.0 & 1.0 & 1.0 & 0.0 & 0.0 \\
\hline PGC061468 & & 0.0 & 0.0 & 0.0 & 0.0 & 0.0 & 0.0 & 0.0 & 0.0 & 0.0 \\
\hline PGC071531 & $-\mathrm{pc}$ & 0.0 & 0.0 & 0.0 & 0.0 & 0.5 & 0.0 & 0.0 & 0.0 & 2.0 \\
\hline UGC03960 & & 0.5 & 0.0 & 0.0 & 0.0 & 0.0 & 0.0 & 0.0 & 0.0 & 0.0 \\
\hline UGC04551 & & 0.0 & 0.0 & 0.0 & 0.0 & 0.0 & 0.0 & 0.0 & 0.0 & 0.0 \\
\hline UGC05408 & $+\mathrm{s}+\mathrm{r}+\mathrm{ph}$ & 2.0 & 2.0 & 0.0 & 0.0 & 2.0 & 0.0 & 0.0 & 0.0 & 0.0 \\
\hline UGC06062 & $+\mathrm{pb}$ & 0.0 & 0.0 & 0.0 & 0.0 & 0.0 & 0.0 & 2.0 & 0.0 & 0.0 \\
\hline UGC06176 & $+\mathrm{pb}$ & 0.0 & 0.0 & 0.0 & 0.0 & 0.0 & 0.0 & 2.0 & 0.0 & 0.0 \\
\hline
\end{tabular}


Table D1: continued.

\begin{tabular}{|c|c|c|c|c|c|c|c|c|c|c|}
\hline Name & "Classification code & Streams & Shells & $\overline{\text { Tails }}$ & Ext. SF & Out. isoph. & $\overline{\text { Dust }}$ & Bars & "Halos & 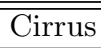 \\
\hline UGC08876 & $+\mathrm{pb}$ & 0.0 & 0.0 & 0.0 & 0.0 & 0.0 & 0.0 & 2.0 & 0.0 & 0.0 \\
\hline UGC09519 & $+\mathrm{d}+\mathrm{wl}$ & 0.0 & 0.0 & 0.0 & 1.0 & 0.0 & 1.0 & 0.0 & 0.0 & 0.0 \\
\hline
\end{tabular}

This paper has been typeset from a $\mathrm{TEX}_{\mathrm{E}} / \mathrm{LAT}_{\mathrm{E}} \mathrm{X}$ file prepared by the author. 